\title{
The Triggering Track-ways Theory
}

\author{
By
}

\section{Kim Shaw-Williams}

A Thesis Submitted to the Victoria University of Wellington

In Fulfilment of the Requirements for the Degree of

Master of Arts

In Philosophy

Victoria University of Wellington

2011 


\section{ABSTRACT}

In this thesis I present a new paradigm in human evolutionary theory: the relevance of track-ways reading (TWR) to the evolution of human cognition, culture and communication. Evidence is presented that strongly indicates hominins were exploiting conspecific track-ways 4 million years ago. For a non-olfactory ape that was a specialized forager in open, featureless wetland environments, they were the only viable natural signs to exploit for safety, orienteering, and recognizable social markers. Due to the unique cognitive demands of reading track-ways, as compared to scenttrails all other animals use to find each other and preferred prey species, social TWR triggered the evolution of a unique faculty for narrative elsewhere-and-when cognition in the hominin mind. Two million years later, this narrative faculty was entrenched enough to enable the rather sudden 'explosion' of co-operative Oldowan Lithic Culture that began at 2.6mya. This cultural adaptation was a highly successful response to catastrophic environmental change. Thereafter selection for encephalization to increase neural capacity to store and co-operatively exploit socio-ecological knowledge gained from the hominin narrative faculty (via co-evolving, increasingly efficient modes of intentional communication) drove all further biological and cultural developments in the hominin trajectory towards H.sapiens and behavioural modernity. 


\section{Contents}

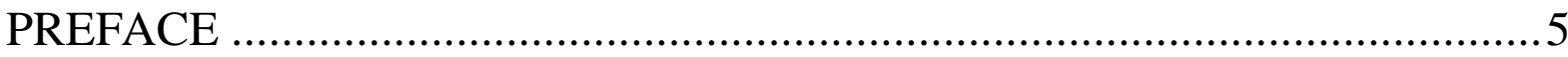

Chapter One: The Triggering Track-ways Theory......................................... 9

1.0 The Uniquely Hominin Cognitive Niche of Track-ways Reading ......................................... 9

1.1 The Qualitative Difference between the Ape and Human Mind-sets................................... 16

1.2 The Narrative Nature of Track-ways as a Natural Sign-System ......................................... 18

1.3 Social Cognition: the Hominin Narrative Faculty (HNF) .................................................. 22

1.4 Summary and Outline of Following Chapters ................................................................ 25

Chapter 2: The Nature of Track-ways Cognition............................................29

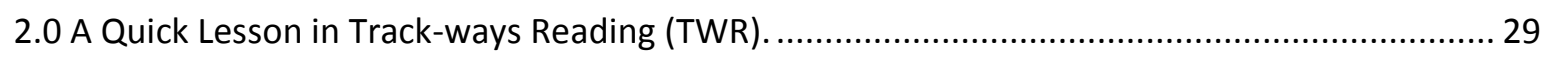

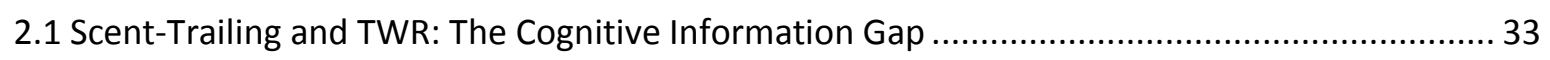

2.2 The Calibration of TWR Cognition: Simple, Systematic, Speculative. .................................. 39

2.3 Mental Applications of TWR Information: How Early Hominin Hunters 'Thought' ................. 44

2.4 The Pedagogy or Cultural Transmission of TWR Skills ................................................. 49

Chapter Three: Oldowan Hunters and Gatherers ..........................................58

3.0 Big Game Hunters or Obligate Scavengers? ................................................................ 58

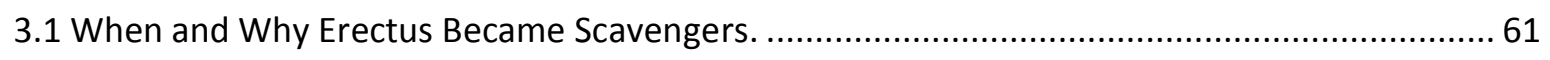

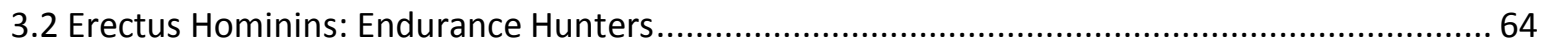

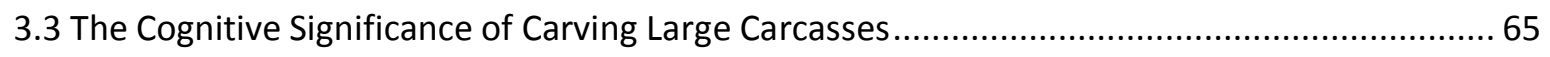

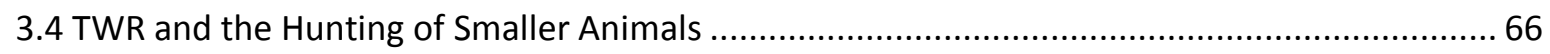

3.5 The Cognitive Competences of Oldowan Hunters and Gatherers ......................................68

3.6 Reasons for Previous Theoretical Disregard for TWR ..................................................... 71

Chapter 4. Social TWR and the Hominin Narrative Faculty ............................76

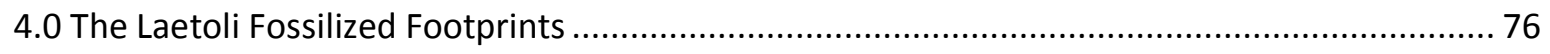

4.1 Physical and Ecological Attributes of the Laetoli Hominins ......................................... 79

4.2 Cognitive Attributes of the Laetoli Hominins ................................................................. 83

4.3 Why Only the Hominin Lineage Entered the Social TWR Niche. ........................................86 


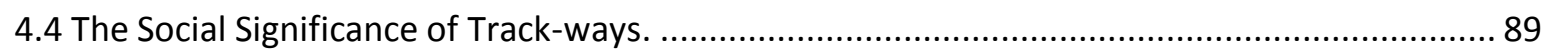

4.5 How Social TWR Increased Hominin Social Complexity .......................................................... 91

4.6 The Initial Entry into the Social TWR Cognitive Niche …........................................................... 93

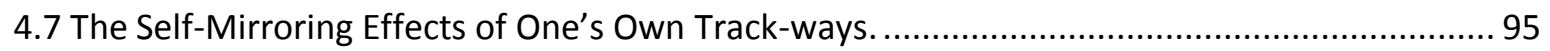

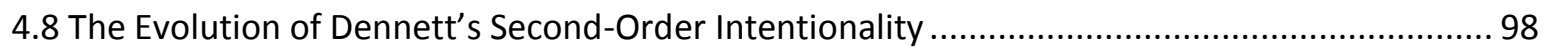

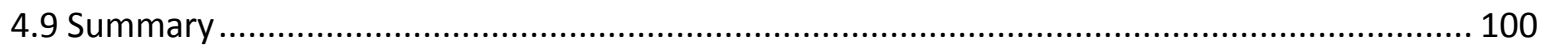

Chapter Five: The Hominin Narrative Faculty and the Cornerstone Social

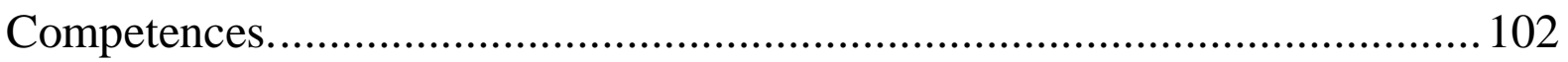

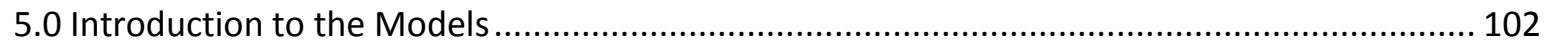

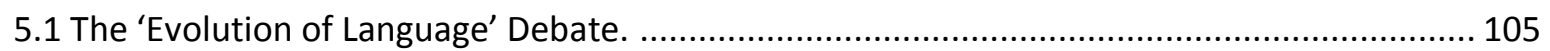

5.2 Overview of the HNF and the Cornerstone Competences .................................................. 107

5.3 The Qualitative Uniqueness of the Narrative Faculty.......................................................... 109

5.4 Developmental and Comparative Evidence of Ontogeny of the HNF. .................................. 111

5.4 Summary and Overview of Phylogeny and Ontogeny of Narrative Faculty............................ 115

Chapter Six: The Hominin Evolutionary Trajectory..................................... 119

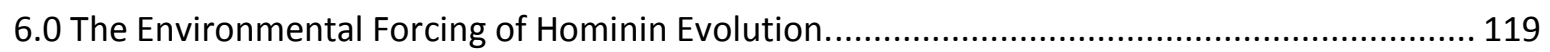

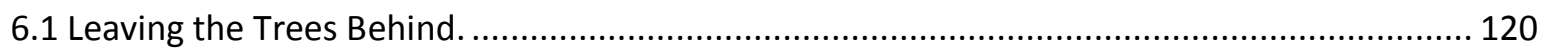

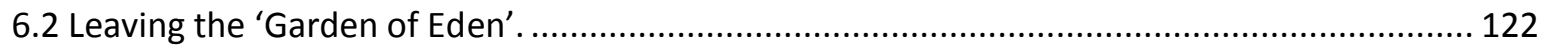

6.3 The Advent of Oldowan Culture and the 'Co-operative Explosion' ........................................ 127

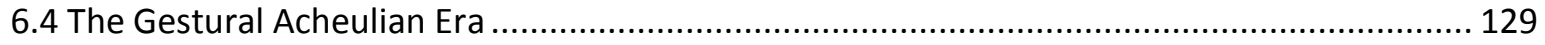

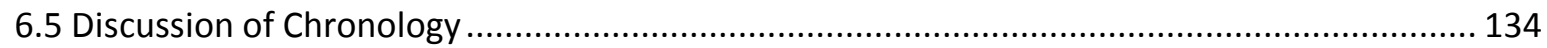

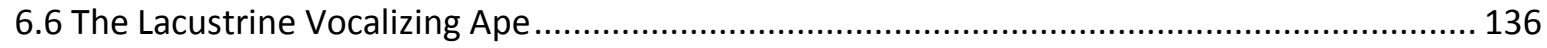

6.7 The Socio-Ecological Effects of Linguistic Communication .................................................. 137

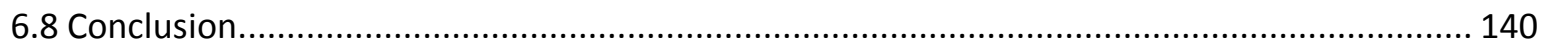

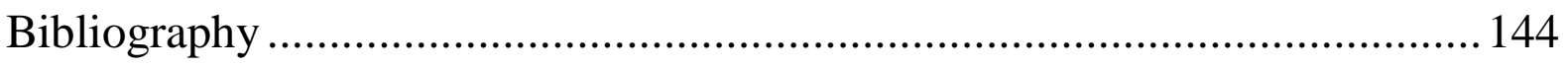




\section{PREFACE}

As a child growing up in the wilderness of N.W. Canada I was encouraged to set snares for snowshoe hares to add to the family meat supply in winter. Hence, like any other huntergatherer child, I had begun to learn track-ways reading skills before the age of six. By the age of eight I was setting leg-traps for small fur-bearing mammals and selling their dried and stretched skins for pocket money. By then I could recognize the footprints of most local species of both small and large mammals, and discern from their track-ways what they had been up to during their daily routines. Most modern humans never need to learn how to read track-ways. But for two to three million years before sedentary horticultural civilizations began to emerge (roughly 10,000 yrs ago) all hominins were hunter-gatherers, and therefore must have begun to learn how to read track-ways from the earliest age possible.

Here is a personal anecdote that I believe begins to capture the cognitive and psychological import as well as the possible evolutionary significance of track-ways reading as a cognitive/behavioural niche. At the age of twelve I once went snow-shoeing ${ }^{1}$ in late winter to investigate a certain local copse of evergreen spruce trees. There had just been a fresh fall of snow the night before, and I wanted to read the tracks in the fine dusting of fresh snow underneath their protective cover because I was looking for new sites for my traps. One purposefully reads tracks at such times (another example is after a period of rain in areas of sand or mud) because all tracks and other traces of target animals to be found must be very recent: one can be sure their perpetrators or 'authors' are still in the territory being explored.

There were tracks of some smaller fur-bearing species, but 'something did not feel right' in the gloom of the small forest ${ }^{2}$, so I retreated from the trees and laboriously climbed a steep, un-forested hill above them for a good view of the surrounding area. After all, it was one of those immensely beautiful, 'pristine' sunny winter days that occurs after new-fallen snow the day before. When I descended back down to the trail I had made on top of the deep soft snow into and out of the spruce grove in order to head for home ${ }^{3}$, I saw the track-way of a very large cougar that had followed my own snow-shoe track-way for a hundred yards or more out

\footnotetext{
${ }^{1}$ Snowshoes are an ancient northern temperate-zone hunter-gatherer invention consisting of strips of leather woven across hooped frames of wood that are strapped to one's boots like skis. They enable one to walk on top of the snow, no matter how deep it is.

2 All hunters will relate such 'sixth-sense' stories, and also attribute this mental state to other animals. In short, it is described as the feeling of 'being watched'. Therefore young Indian hunters were admonished by their elders to never look directly at the target animal until ready to fire their arrows.

${ }^{3}$ One naturally back-trails when snow-shoeing in fresh snow, because it is much easier to travel on the already packed-down snow under one's former prints.
} 
into the open before returning to its lair somewhere in the trees. I could see by its prints where it had paused, turned to the side of my trail that faced the steep hill, and then padded back through the snow into the trees.

One story that I gleaned from the combination of our track-ways was that it had sensed my entry into and departure from the trees and had checked out my scent trail to make sure that I had left the vicinity, pausing when it saw me as I laboured up the steep hill, then returned to its lair relieved. Another more dramatic story suggested by the combination of our track-ways might have been that it was hunting me because it was unusually hungry due to that year's prolonged and very cold winter, but gave up because a) I was already well above it on the hill and out in the open; b) the deep soft snow put it at a physical disadvantage; c) I was human and therefore dangerous to attack. Since I knew cougar attacks on humans were extremely rare, I quickly discounted that story.

Even so, the sense of 'self' as a young human alone in the wilderness without a gun or a hatchet came to my mind very strongly indeed. For I could not help 'seeing in my mind's eye' my past-self leaving the trees, and almost immediately afterwards, a very large and very hungry cougar finally giving up its ambush waiting-game somewhere in the forest gloom (cougar are ambush predators in the main) and rushing out of those same trees, nose to the snow, before it visually ascertained that my past-self was already half-way up the steep hill above.

What I was mentally doing in this track-ways-reading event, in essence, was imagining 'being-in-the-mind-and-body' of both characters in a past-narrative of this close encounter between two agents, the unseen cougar's past-self and my own past-self. And all this mental activity was based on my visual analysis of the purely physical signs made in the past in the snow that had recorded our track-ways. Like all mammalian predators the cougar was following my scent-trail till it saw me, and like all hominin predators I was cognitively exploiting track-ways.

As a trapper I knew that no animals would ever come near my traps until the human scent I could not help leaving behind while setting them had disappeared, always within a few hours. So the very next day the cougar could have re-crossed my snow-shoe trail again without gaining an inkling that I had ever been there. As a tracker I knew that for me or any other hunter the above track-ways story (and therefore the cougar's possible proximity) would have been readable until the next snow-fall or the spring thaw, perhaps a month or more later. I 
also knew that no other species on the planet used visual analysis of track-ways to find and/or hunt each other or other animals. The result of this knowledge, plus my awareness of Darwinian evolution and 'how humans must have evolved from chimps', was a 'eureka' moment a couple of years later, on another expedition looking for new trap-sites.

It suddenly occurred to me that our ancestors might have been reading the stories told by track-ways since before we became human. My next idea was that the stories I was reading every day told by footprints and track-ways equated with the stories told by printed words and sentences used in the books I 'devoured' every winter evening (neither electricity nor television had reached our wilderness area back then). I realized printed words could be viewed as 'thought-prints'.

The 'eureka moment' really happened then, because I realized that when hominins became smart enough to be able to fully read track-ways, they must have started inventing their own track-ways, made up of agreed upon depictive signs like trail markers (stone cairns and so on) for others to follow physically. As time went on the gestures of sign languages, the vocalizations of linguistic languages and then the printed word were invented in order to tell stories for other humans to follow mentally.

I have recounted the above auto-biographical material for two reasons:

1) So my readers will know I speak from experience about learning and applying tracking-ways reading to hunting and gathering skills, not just as an anthropological or ethnological observer. In much of what follows, I shall be drawing on a life-time's experience of tracking animals when trapping or hunting.

2) My cougar story is seminal to my evolutionary narrative or thesis: I shall be referring back to it quite often in this paper.

Apart from track-ways reading skill being an absolute necessity for a non-olfactory ape to be such a highly successful predator, there are three other major aspects of what it entails to 'be a human', as compared to other animals, that I learnt from living in the wilderness, especially by spending a month or so without contact with other humans.

In the first place, no fur-less, fangless, clawless human can manage to survive alone in the wild for any length of time without access to certain tools: sharp cutting edges, some kind of digging implement, a club, spear or other projectile weapon, containers or packs for carrying equipment and foraged resources, and protective clothing and/or shelter of some sort in 
colder climates. These items would often have been manufactured by older more skilled kinfolk. Hominins had to have been tool-makers and reciprocally co-operative from near the very beginning of our evolution.

Secondly no human can survive in the wilderness for very long without hunting and killing animals because all plant-life is seasonal, often poisonous or indigestible without prolonged processing (usually requiring fire), and demands co-operative, complex preservation techniques in order to provide dependable nutrition over time. Animals of course survive and can be exploited for food all year round (especially fish and shellfish), and their flesh is always highly nutritious, even when raw. As far as scavenging is concerned, it is both too dangerous and far too 'hit-and-miss' to be depended upon for survival on a day-to-day basis ${ }^{4}$. So we had to have been hunters and fishers (as well as seasonal gatherers), to at least the same degree as chimps are, from the beginning of our lineage's evolution.

And last, but very definitely not least: no human can survive mentally for very long without some other human to tell his or her stories to. The human brain never stops creating stories about the self and other entities interacting in the past and the future (even when asleep). If there is no one else around to talk to (or communicate with in other ways) one quite soon finds oneself talking and singing out loud to oneself. After only a few weeks the mind begins to create imaginary 'companions', especially during bad weather when long periods of inactivity encourage larger amounts of time spent in the lucid dream state.

For some people these imaginary 'companions' can become extremely upsetting, powerful mental phenomena. I personally coped with this problem by always carrying a pen and a notebook to describe my daily experiences in (for an imagined future audience in the real world, although I knew my future self would probably be the only person interested in reading about them). Even so, a month alone was definitely long enough to become desperate for some form of mental exchange with other humans.

Barry Crump, a renowned New Zealand bushman and famous author (now deceased) once wrote: "If you spend any longer than three months alone in the bush don't bother coming out". Schizophrenia was described in one very early psychological treatise I once read as "a

\footnotetext{
4 Of course, when we became the most successful, dangerous predator on the planet we could take the best meat from other predators whenever we had to. They got the remains, and what we left behind from our kills as well. I bet this is how our somewhat symbiotic relationship with dogs and cats began.
} 
disease often suffered by lonely shepherds". We humans cannot function without being narrative-minded and therefore have to be eusocial story-telling creatures. We also cannot function without an up-to-date self-narrative incorporating personal past memories and future plans, as the pathologies of amnesia and Alzheimer's disease clearly exemplify.

Hence I suspect our very earliest ancestors were already beginning to be narrative-minded. We could in fact be said to be Homo narrativans, and I believe we became story-thinkersand-tellers because we started reading the stories told by track-ways at the very beginning of hominin evolution.

\section{Chapter One: The Triggering Track-ways Theory}

\subsection{The Uniquely Hominin Cognitive Niche of Track-ways Reading}

Track-ways reading (TWR) is the goal-directed visual analysis of patterns of indentations inadvertently left behind in suitable substrates (sand, soft clay, mud, volcanic ash, new-fallen snow) by the physical passage of all land-based animals, including, of course, our own species. For example, consider the trails of foot-prints made by humans, dogs, birds such as sea-gulls, tire-marks of various vehicles, impressions made by people fishing, sun bathing, building sand-castles and so on in the sand that you may have noticed when you last visited a beach by a river, lake or sea. Other physical traces are also taken into account, depending on the kinds of environments in which one is tracking: faecal matter, bent grass, turned-over leaves and up-turned pebbles, torn moss, blood spots, browsed foliage, and so on.

Many people are not aware that hominins are the only species lineage on this planet that has ever taken notice of track-ways, including our closest genetic cousins, chimps and bonobos. All other terrestrial predators and scavengers ${ }^{5}$ (such as canids, felids, and hyenas) follow scent trails on the ground and scent transported by air-currents until within visual or auditory reach of their targets. Others are ambush predators as well, like leopards and cougars. Air borne or water borne predators and scavengers use panoramic vision (eagles, vultures), scenting (sharks) or echo-location (bats, cetacea).

According to orthodox niche construction theory, in the case of more complex and behaviourally flexible organisms, entering a new cognitive/behavioural niche can lead to phylogenetic and ontogenetic changes in genome, morphology and behaviour further along

\footnotetext{
${ }^{5}$ All predators sometimes scavenge, and scavengers will prey on very young or old animals as well.
} 
the evolutionary trail (West-Eberhard 2005) (Jablonka 2006). The end result can be one or more new species. If that cognitive niche is unique on this planet then surely it follows that some aspects of the neuro-cognitive capacities and resultant behavioural attributes of those species may also be unique on this planet. Therein lays my view of the fundamental relevance of TWR to the evolution of human cognition, culture and communication.

I take the strong view that no true hominin species would have evolved if pre-hominins had not entered the TWR niche. My over-all reasons for this view are these: (a) track-ways are very different in important ways from scent-trails, which all other terrestrial mammals use to keep track of their own and each other's whereabouts and search out targeted animals (and plants); and (b) those differences make track-ways reading far more effective than scenttrailing for achieving those purposes. Therefore positive selection for the unique cognition demanded by track-ways exploitation immediately began to take our ancestors into a correspondingly unique cognitive realm.

To summarize my theoretical stance on how the first true hominins evolved:

1) The definitive question in the hominin evolution debate is what gave pre-hominins the unique cognitive capacity for such an unprecedented cumulative cultural adaptation as proto-hominin Oldowan Lithic Technology.

2) The selective trigger for the evolution of that unique cognitive capacity must have been entering a unique cognitive/behavioural niche that in turn demanded a unique kind of cognitive development.

3) That unique niche had to have been entered deep in our evolutionary past because the unique cognitive capacity it triggered selection for had to have been genetically and neurologically entrenched well before the abrupt emergence (and ongoing continuity ${ }^{6}$ ) of Oldowan Culture (2.6 Ma).

4) The emergence of Oldowan Culture was caused by an adaptive cliff, created by catastrophic environmental events just after 2.8 mya: a sudden north/south polar reverse, associated with a peak in volcanic and earthquake activity in the East African Rift and the beginning of extreme glaciations in the Northern Hemisphere.

5) This proto-hominin cultural adaptation to those catastrophic environmental effects, enabled by their unique cognitive competences, constituted a powerful fuel for success that engendered selective forces for more of the cognitive capacity

\footnotetext{
${ }^{6}$ I have inserted continuity as a feature here because cut-marked bones dated 3.4 mya have recently been found.
} 
for such adaptations; for these were highly effective behavioural/cultural adjustments to on-going environmental change throughout the Late Pliocene.

6) These cultural adaptations created more social and ecological complexity which positively fed back into individual and group selection for more cognitive capacities as well; the need for more high-quality nutrition to fuel a larger brain needed for these cognitive capacities demanded further socio-ecological adaptations in turn.

7) This co-evolutionary spiral caused rapid encephalization, together with compensatory morphological and life-history changes (plus a full entry into the social co-operative breeding carnivore niche). This process caused the first true hominins species habilis and erectus to evolve by around 2 mya, if not before, and established the extreme disparities between hominin and ape cognition, culture and communication by 1.6 mya.

8) This gene-culture, niche construction co-evolution, together with increasingly dramatic climactic fluctuations (glacial cycles) after the mid-Pleistocene, explains all further biological and cultural developments in the hominin lineage. Those developments resulted in neandertalis and sapiens, linguistic communication by around $300 \mathrm{ky}$, and finally behavioural modernity by $100 \mathrm{ky}$.

I believe entering the unique cognitive niche of TWR was the selective trigger that led to the evolution of the first true hominins habilis/erectus, with the help of other pre-adaptive morphological and cognitive/behavioural traits, of course. Some of these traits were already possessed by all great apes, and I turn to them later in this section. Others were possessed only by pre-hominins because they were already specializing in a different socio-ecological niche from all other great ape species.

I refer to the peculiarities of the socio-ecological niche of the very early (5.8 to 4.4 mya) prehominins Ardipithecus kadabbas and ramidus ${ }^{7}$. Some theorists are now suggesting they were the last common ancestor to hominins and australopithecines (McPherron, Alemseged et al. 2010), and that chimps are an earlier side branch that headed down their own adaptive trail

\footnotetext{
${ }^{7}$ We now know a great deal about their paleobiology due to several papers just recently published by a multidisciplinary scientific team (for example Suwa et al 2009). They have been working over the last decade on fossils from several individuals (and the nature of the environment they lived in), of which one was fortuitously virtually complete.
} 
around 6 mya [(Zollikofer and Ponce de León) to be published]. I support their view: the ardipithecines occupied the adaptive plateau from which true hominins evolved.

The great-ape Ardipithecus ramidus was fully bipedal (no knuckle-walking), but had an extremely opposable big toe still useful for grasping tree $\operatorname{limbs}^{8}$. Male canines were already very much reduced compared to those of chimps, indicating that male aggression was not important as a sexual signal. Their post-canine molars were also much smaller, indicating they were omnivorous, and they foraged in patches of lightly forested flood-plains (predominately palm-trees) and open wet-lands within upland closed forests (Suwa, Kono et al. 2009), (WoldeGabriel, Ambrose et al. 2009). What this evidence indicates is that they still nested in trees at night, but foraged daily for semi-aquatic flora and fauna.

At about 4.39 mya a huge volcanic event deposited enough ash to destroy evergreen tropical forests throughout the East African Rift. This event also cut off the outlet of the Omo River from the Turkana Basin to the Indian Ocean due to massive lava flows. I have presented these details here because similar events throughout the Late Pliocene and early Pleistocene played havoc with wetland resources that I believe were still the staple diet of hominins, thus forcing some populations into habitual acquisition and butchery of large mammals. Hence my thesis is complemented by the environmental variability hypothesis (Potts 1998).

I hold the above Mid-Pliocene environmental catastrophe is the beginning of the hominin evolutionary story, because it forced some populations of ramidus to adopt a completely terrestrial life-style, living and foraging in large expanses of open semi-aquatic highland environments. The major pre-Oldowan morphological and behavioural traits that consequently had evolved by around a million years later (3.5 mya) were:

1) Full and obligate bipedalism, with very humanoid feet (completely nonopposable big toe as proved by Laetoli Fossilized Hominin Footprints)

2) Two free hands and a gluteal morphology (un-chimp-like buttocks providing fulcrum for weight transmission to shoulder and arm for greater throwing force) from habitual bipedal wading, coupled with a precision grip and pliable wrists (originally for climbing but also useful for foraging under water). These features enabled hard, accurate throwing and clubbing and were a pre-adaptation for stone knapping.

\footnotetext{
${ }^{8}$ Their feet would have been useful for walking in the soft mud of swampy substrates, but would have made for slow bipedal traveling.
} 
3) The correspondingly free-handed physical ability to carry things (for example, clubs/stones for hunting and defence) for long distances (unlike knucklewalking chimps) and therefore have longer behavioural sequences in mind than chimps (Osvath and Gärdenfors 2007).

4) Decreased sexual dimorphism and an incipient form of co-operative breeding consisting of communal/kinship care/feeding/transportation of the young, due to habitual foraging in watery places, in which the very young could not safely forage for themselves (the young of baboons, clinging to their mother's fur, often drown when water-ways in swamps are being crossed by quadruped baboon troupes).

5) Morphological features of aquatic or semi-aquatic mammals: loss of hair, copious sweating capacity, tears, loss of air sacs in the throat (present in all other great apes including australopithecines), fatty mammary tissue (breasts), breathe control [(Verhaegen, Puech et al. 2002; Cunnane and Stewart 2010) foreword by Tobias]

6) More than an ape-like capability to manufacture simple tools, and to do so in a much more anticipatory manner. This view is non-speculative given very recent evidence of cut-marked bones from 3.4 mya (McPherron, Alemseged et al. 2010).

7) The obligate, habitual exploitation of conspecific track-ways (including their own old track-ways), both to ensure safety in boggy swamp-land substrates and enable optimal navigation in featureless open environments where the predominant vegetation was very tall tropical swamp-grasses and reeds ${ }^{9}$ (see illustrations next page. The swamp is a seasonal swamp, or in other words a lightly forested floodplain. From Photo Share, flickR website)

In sum then: in this thesis I am presenting a new paradigm by focusing on a uniquely hominin routine cognitive behaviour called 'track-ways reading' (TWR), engendered by the fully terrestrial open wetland/floodplain socio-ecological niche occupied by post-ardipithecan hominins. I argue that entering the TWR niche selectively triggered the co-evolutionary development of the extreme disparities between human cognition and that of other great apes.

\footnotetext{
${ }^{9}$ It may help my readers to think of the words of an old song: "The grass (corn in song) grew as high as an elephant's eye" to understand the difficulties in navigation our ancestors would have had foraging in such environments, given that they were apes with extremely poor olfactory perception.
} 

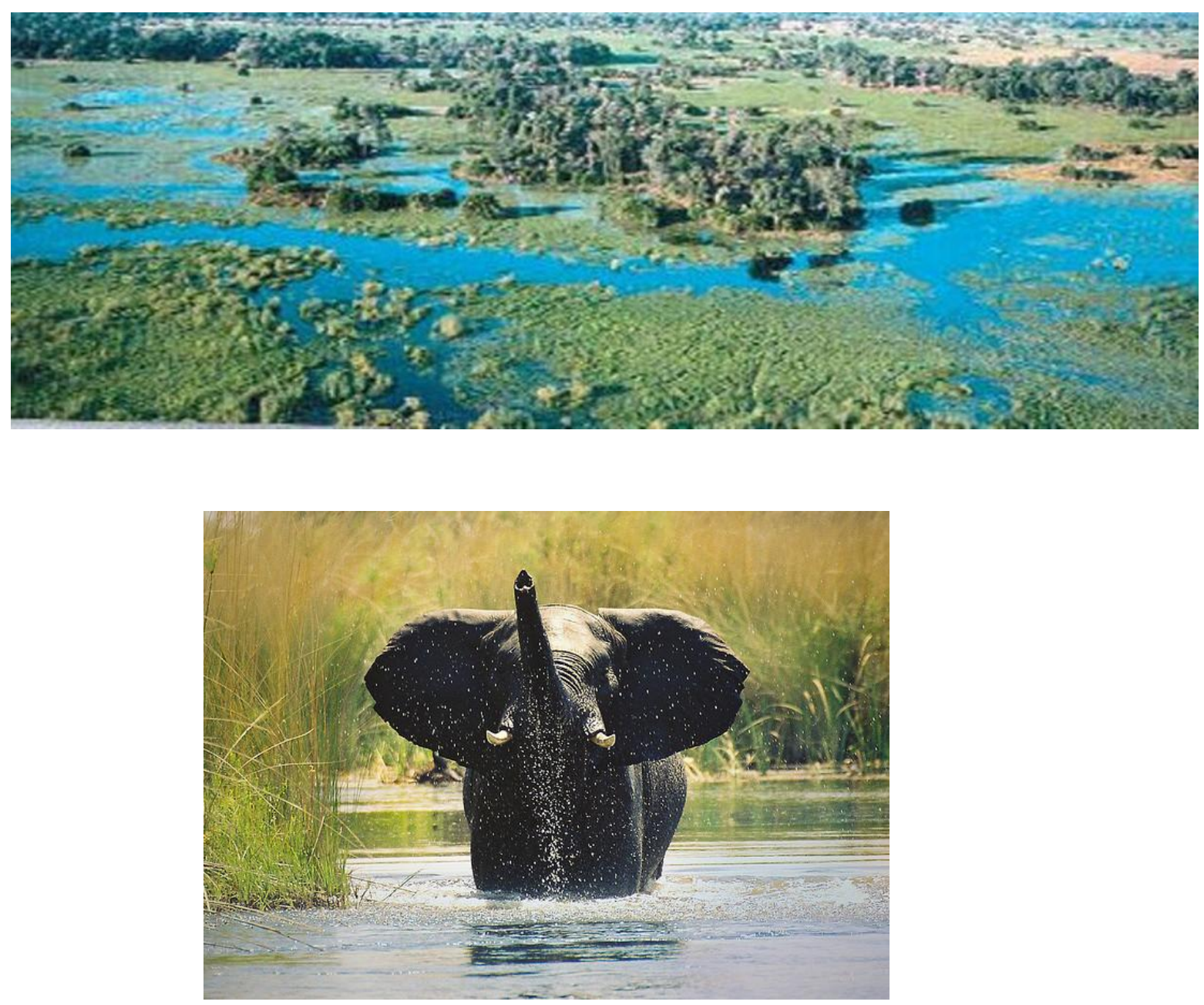

I hold these disparities are caused by a basal qualitative difference between the ape mind-set and the human mind-set. Only human brains have a neurally entrenched faculty for fully decoupled or elsewhere-and-when cognition, travelling in "the theatre of the mind" through imagined narratives made up of participant/event scenarios in the past and the future (Suddendorf and Corballis 2007). This faculty enables mental time-travel, for instance.

The other vital capacity this elsewhere-and-when cognition confers upon humans is the ability to take "the first-person and third person point of view" ((Tomasello, Carpenter et al. 2005). This enables us to imagine 'being-in-the-minds-and-bodies' of other agents and entities, as well as past and future selves, in "the theatre of the mind". The "first-person" view is a meta-representation of oneself (and therefore of others as "third persons") as an intentional agent travelling from the past into the future. This unique social competence 
makes us consummate mimics, mind-readers and folk psychologists, and begins to be expressed by all normal humans between the age of three and four, when childhood amnesia stops and pretend play with peers begins (Atance and O'Neill 2005).

Tulving (Tulving 1985) coined the term autonoesis for this unique autobiographical memory or 'self-knowing' capacity, and has more recently stated (2001 for review) that this attribute is the key cognitive capacity behind all hominin culture and communication. Therefore one cannot explain the evolutionary trajectory of human cognition without explaining how autonoesis evolved in our forbears. Without this unique cognitive capacity for a detached or objective view of the self, elsewhere-and-when cognition about other entities and the world at large could not exist. As we will soon begin to discover (section 1.2), this is fundamentally the cognition that is required for full-fledged TWR skills.

My explanation for the evolution of autonoesis is that habitually following one's own old track-ways (as well as those of intimate others) for safety and orienteering triggered the evolution of autobiographical narrative ${ }^{10}$ memory. I think the initial ability to read track-ways must have evolved in the psychological realm of iterated physical separations between socially intimate conspecifics: that is, in the social domain proper. When extractive foraging, one is always leaving or returning to one's most important others: therefore I class this kind of TWR as social TWR.

Only using TWR for finding other animals has ever been considered by other evolutionary theorists [(Liebenberg 1990) for example]. However they restricted TWR's evolutionary relevance to the advent of behavioural modernity, or subsumed it under the umbrella of cognition required for all earlier HG extractive-foraging techniques. I am alone in suggesting beginning to read our own and intimate others' track-ways is what triggered the evolution of uniquely hominin cognition.

Finally, we need to discuss great ape social competences: I hold that only some great-ape species could have entered the TWR niche, because apes are excellent visual pattern readers, with very poor olfactory perception compared to non-arboreal mammals. They are also very smart mammals, with comparatively good here-and-now social learning skills [(Bates and Byrne) in press]. Certain universal great ape here-and-now cognitive competences possessed

\footnotetext{
${ }^{10}$ Tulving (1985) also coined the term episodic (knowing who and when) for what I prefer to call narrative or autobiographical memory, to differentiate it from semantic (knowing what) and procedural (knowing how) memory, which all other animals have of course (think of cognitive maps of home territories). An episode is merely the shortest possible narrative.
} 
by $A$. ramidus had to be the platform for the elsewhere-and-when narrative imagination required for social TWR skills. I will argue these were the social competences of here-andnow exact imitation and theory of mind/intentionality-reading, as exhibited by chimps and human toddlers under the age of three (Call, Hare et al. 2004; Call and Tomasello 2008; Tomasello 2008).

In my view this explains why the hominin narrative faculty is overwhelmingly a faculty for social cognitive skills. Later on hominins began habitually reading track-ways of other animals and all other natural signs (due to environmental forcing, after 2.8 mya) using their simple social TWR skills and this qualitatively unique narrative social faculty. Only then were they fully on their way to becoming the most successful eusocial predators on the planet.

In the next section I list the disparities between ape and human minds, and claim they are due to a qualitative difference: a unique faculty I call the hominin narrative faculty (HNF). Section 1.2 will then introduce us to the narrative nature of track-ways as natural signs, and their three major differences from scent-trails. The following section then presents my reasons for arguing that this faculty could only have evolved in the social navigation domain. The final section summarises earlier material very briefly, and then outlines the structure of the thesis as a whole.

\subsection{The Qualitative Difference between the Ape and Human Mind-sets.}

After more than 40 years of studying chimpanzees and bonobos in the wild and by laboratory research, plus extremely prolonged enculturation of apes such as the famous Kanzi (who is now 30 years old), what has been discovered is that they sometimes appear to incipiently exhibit many human cognitive/behavioural traits. However, they also exhibit these glaring disparities:

1) Unlike us they are overwhelmingly cognitively restricted to communicating and acting only with the daily here and now in mind.

2) They make simple tools but do not use tools to make other tools.

3) While they can truly imitate conspecifics for the sake of attaining immediate rewards in the associative here-and-now, they immediately revert to mere goaldirected emulation if a quicker route to the reward becomes apparent (perhaps because they are extremely competitive). They almost never indulge in pretend play 
formatted on participant/events that have happened or could happen in the elsewhereand-when.

4) They almost never exhibit intentional pedagogy (mothers directly showing their young how to crack nuts has only been observed twice in 40 years) or auto-rehearsal (imitation of skilled conspecifics when they are absent) necessary for fast-track transmission of skills (chimps take several years of trial and error learning to achieve nut-cracking expertise).

5) Even highly enculturated apes with some understanding of symbols or gestures make only very simple two-symbol requests, like human toddlers, and cannot use symbols to tell stories about events not occurring in the here and now. They do not point demonstratively, or even understand demonstrative pointing to third entities by their human caretakers.

6) They exhibit no moralistic punishment (they don't have cultural norms)

7) They exhibit very little co-operative sharing, trade (the only exceptions are a 'tolerated theft' version of sharing of meat and reciprocal grooming) or collaborative action towards mutual rewards.

8) They do not 'read' or cognitively exploit the natural signs we call footprints and track-ways, not even those of their own species when hunting other chimps.

I argue that the last disparity is the major evolutionary reason the first disparity exists, and the first disparity is the over-all cognitive reason why the following six disparities exist. Apes are overwhelmingly restricted to here-and-now cognition whereas even the default state of the human mind/brain is characterized by narrative elsewhere-and-when cognition. Neuroimaging work over the last decade has proved there is a core brain structure that supports this narrative mentalizing (Addis, Wong et al. 2007; Schacter and Addis 2007; Addis, Pan et al. 2009; Schacter and Addis 2009; Spreng and Grady 2010). I call this faculty the hominin narrative faculty (HNF); it is so entrenched the human mind-brain is truly a "narrative machine'.

In the next section we will discover track-ways as natural signs demand this narrative form of cognition, about temporal and spatial relationships between characters and events occurring in the elsewhere-and-when, in order for them to be very useful for navigation. 


\subsection{The Narrative Nature of Track-ways as a Natural Sign-System}

Gordon Hewes (Hewes 1994) is the only other theorist ${ }^{11} \mathrm{I}$ have discovered that has directly mentioned the fact that track-ways tell stories, in a manner similar to languages, about agents acting towards goals over time:

“...the total record left by some animal's tracks and other trailside signs within a normal time frame (such as the main part of a day) might be regarded as a kind of unintentional narrative, in which the tracker develops a profile of the kind of creature he is pursuing. The human tracker's cognitive achievement in forming such a "narrative" does not seem inferior to that required for transmittal of a comparably accurate verbal report...the process by which a nonhuman predator follows a scent track to its quarry is much simpler, depending mainly on pursuit of increasingly strong stimuli in the same mode." (my emphasis, p 142).

So if one follows it for long enough any track-way can naturally tell a story about 1) where an absentee agent or 'author' has been and what it has been up to in the past; 2) where it might be and what it might be doing in the present; and 3) where it might be and what it might be doing in the future.

There are three basic differences between track-ways and scent-trails. Firstly, track-ways are immediately and inexorably directional because foot-prints physically point to where their author was heading when they were made. A dog following a scent-trail will often head away from the animal that made it until it perceives that the scent is getting weaker instead of stronger. Therefore track-ways reading is immediately more efficient than scent trailing for finding a target animal. Track-ways also appear to have an inherent intentionality, or goaldirectedness, due to their directionality.

Secondly track-ways are far more durable than scent-trails. If a scent-trail can be detected at all, it is worth following, for its author cannot be very far away. Track-ways can last for days or weeks instead of just a couple of hours or so. Therefore choosing which track-way of a targeted animal to follow is arbitrary and depends upon how 'fresh' it is, or how recently it was made. The tracking mind is continually being goaded into thinking about time, into

\footnotetext{
${ }^{11}$ Two other theorists have mentioned that footprints as natural .indexical (indicatory) signs may have given us the ability to "understand the symbolic signs of language":Calvin, W. H. and D. Bickerton (2000). Lingua ex machina : reconciling Darwin and Chomsky with the human brain. Cambridge, Mass., MIT Press. But they did not recognize TWR's import as an ancient, uniquely selective cognitive niche.
} 
decoupled elsewhere-and-when cognition. The scent-trailing mind remains locked into hereand-now associative cognition.

Thirdly, track-ways are combinatorial. Scent-trails are not at all combinatorial because any stronger scent will instantly make an older, weaker scent imperceptible to a scent tracker. Several track-ways can overly each other and still be discernible: this means that spatial analysis of the order of their authorship supplies combined narratives about a conspecific $\mathrm{x}$ meeting up with another conspecific $y$, whether they mated or fought with each other, whether they travelled together after that and so on. Combinations of tracks of different species are also important. For example, the crossing of a targeted track-way by a nocturnal species can tell the tracker whether the target track-way was made today or yesterday, depending on whether the nocturnal animal's prints are over or under the targeted prints. Again, we see how the tracking mind is goaded into thinking about time.

The combinatory narratives told by track-ways are recursive in the sense that:

(1) Complex narratives are comprised from a large but limited array of atomic elements: recognizable track-ways of individuals or of species

(2) The 'meaning' of the complex narrative depends on the presence and arrangement of the individual atomic elements (as with words and sentences). By presence and arrangement I mean the following:

a) Given (say) a meeting between track-ways ( $\mathrm{x}$ ) and (y):

(b) If any footprint of ( $\mathrm{x}$ ) is overlying any footprint of (y) then we can know (x) was travelling behind $(y)$ at that moment in time.

If (say) the track-ways part and we decide to follow (x) because it might therefore be fresher than (y), and yet later we meet (y) again, and this time (y) overlays (x):

(c) Now we know (x) and (y) were wandering along in the same general direction during the same part of the day or night, but at their own speed, individually pausing to do various things.

(d) Since we would already know what (x) had been up to, we might then back-track (y) to see what that particular individual had been up to as well. 


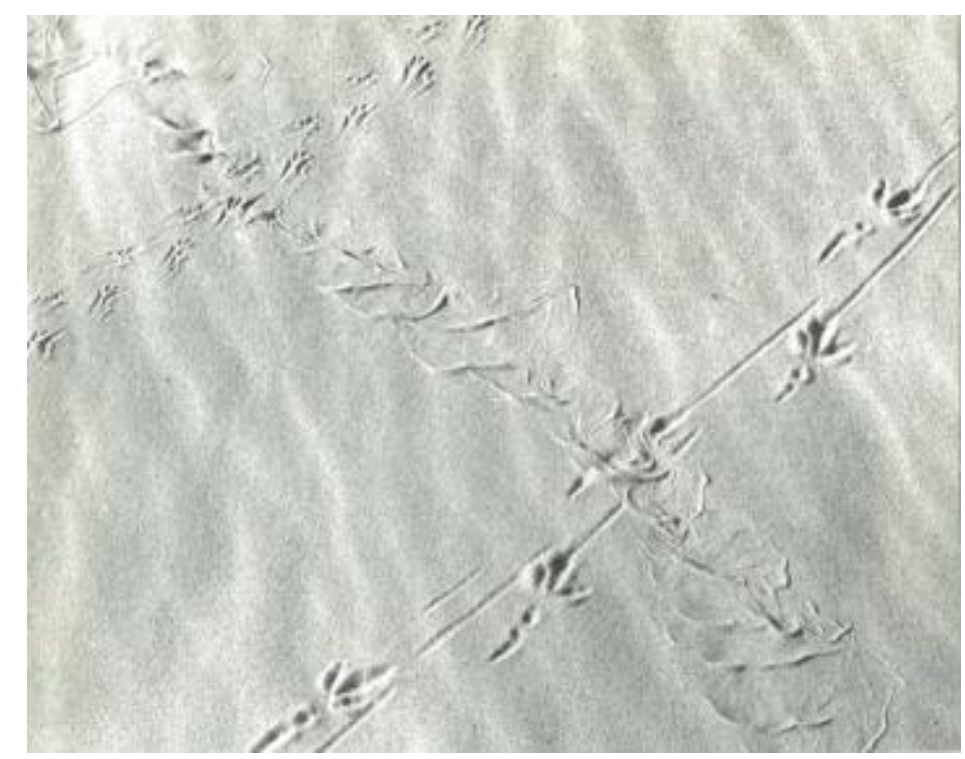

"Having established who was there, the next problem is when and then why. Tracks may provide information obtainable in no other way about what actually happened. This photograph demonstrates time sequence: the Adder, crawling over an earlier Crow track, was in turn succeeded by a Natterjack Toad (top left)".[ (Ennion and Tinbergen 1967) p. 16]

(3) Hence individual narratives can be embedded within, and as a proper part, of more complex narratives.

(4) There is no fixed upper ceiling of complexity.

Hence there is a discrete infinity of track-ways narratives out there in any suitable environment to be read, just as there is in the symbolic signs of natural languages, both morphophonetic (words) and orthographic (writing). And the information supplied by various combinations of them can be useful for HG (hunter-gatherer) foraging and social interactions at any point in the future, analogously to human gossip, hunting stories, the daily newspaper, and so on.

The upshot is that even at the most basic level of skill TWR is far more energy-efficient, dependable and informational than exploitation of scent trails as a means of foraging or social navigation, especially in open and therefore windy lacustrine environments where waterlevels are continually changing. All the paleo-archaeological evidence we have indicates such 
environments were preferred ${ }^{12}$ by our earliest ancestors (White et al 2009, Plummer 2004 for reviews).

Some environments of course are not conducive to TWR. Examples are rocky riverbeds and outcrops in mountainous areas or intertidal sea-side zones, frozen crusted snow in winter, leaf litter and hard packed-down game trails in deep, closed forests. This is especially true for target species with foot-pads. Hoofed animals however still tend to leave marks if one knows what to look for: scrapes on rocks, recently overturned leaves and pebbles with their underside still damp, bent grass, and torn moss and so on.

Track-ways are always useful, no matter how old, for learning about the normal behaviour and daily routines of the animals that made them. So reading track-ways narratives throughout the day whether hunting or gathering is a 'second nature' cognitive behaviour for all members of an HG band. The track-ways of other humans are the most important trackways of all, either for personal socio-psychological reasons, or because strangers might be competing for the same resources. Every Kung San child knows their mother's footprints from all others in the band by as young as the age of four without any directed teaching (Thomas 1963).

There is very good evidence (presented beginning of chapter four) that hominins were exploiting conspecific track-ways to some degree 3.6 million years ago and therefore probably well before, albeit at a quite simple cognitive level. Given (a) the uniquely narrative and information-laden structure of track-ways as a system of natural signs; (b) this very early entry into the TWR niche; and (c) TWR's necessity for a non-olfactory ape to later become such a good exploratory predator; it is not surprising we evolved into the uniquely narrativeminded ape.

In short, tracking cognition is all about narrative mental time-travelling, based on imagining being 'in the mind-and-body' of the author of the track-way being followed. We will gain a good understanding of this in chapters two and three, where we will be discussing the way TWR narratives are used for extractive foraging. In chapter four we will turn back in evolutionary time to our ancestors' initial entry into the social TWR niche, for my readers will by then have fully understood why social complexity was so heavily amped up when they started exploiting their own and each others' track-ways for safety and orienteering. In

\footnotetext{
${ }^{12}$ They still were preferred by most prehistoric and modern HGs, but most have been displaced by modern civilization into marginal resource-poor areas.
} 
the next section I present my reasons for arguing that the evolution of the hominin narrative mind was triggered initially by social TWR.

\subsection{Social Cognition: the Hominin Narrative Faculty (HNF).}

The eco-philosopher Paul Shepard (Shepard 1996) has come closer than any other philosopher or evolutionary theorist to directly pre-empting my 'triggering track-ways theory':

"The human mind came into existence tracking, which for us creates a land of named places and fosters narration, the tale of adventure. Perhaps the quest began as food search. But in scrutinizing the details of the potential prey, competitors, and predators upon ourselves, and all the signs they leave, it seems more abstract, like scientific curiosity, communicated in art and narrated in myth...The whole sequence of brain and mind evolution by attention to animals constitutes a unique twist in the primate obsession with the self and society". (my emphasis, p.78)

My major disagreement is with his extractive-foraging 'food search' version of the hominin TWR story, and is based on the following considerations:

(1) The crucial importance of social relationship skills to the relative fitness of individuals negotiating with the complex social structure of fission-fusion ape societies is unquestionable.

(2) Pre-hominins (including one's self, and this fact is crucial to my story) would have been leaving recognizable footprints behind everywhere they went in the mud and the sand of open wet-land environments.

(3) Chimps hunt chimp strangers (and colobus monkeys) and forage quite successfully without using track-ways in any way. In addition to visual panoramic searching they merely listen for calls and foraging noises and sniff at old faeces and nests of stranger chimps when hunting, and use their cognitive maps of their home territories and well-defined game trails for general foraging purposes.

(4) Therefore our ancestral entry into the TWR niche probably began in the social domain, not in the search for target animals, but for safe passage and orienteering through difficult swampy and featureless terrain.

(5) As the narrative faculty necessary for increased TWR skills co-evolved, social TWR became useful for searching for lost kin-folk, one's own way back to the safety of the band when returning from foraging expeditions, and finding out what everybody else had been up to when they were out of one's sight. The first other 
species' footprints to become salient were probably those of local dangerous predators (as in my cougar story).

Humans are self-obsessed, and I argue this hominin self-obsession is at the core of the unique hominin narrative faculty for 'mind-and-body-hopping', time-travelling elsewhere-and-when cognition. The opposite side of the coin to self-obsession is a corresponding predilection for elsewhere-and-when cognition about other selves: who's who in the social structure, where are they, what are they up to, how do I find them, what will they want from me when I get there, and so on, (let's be honest) ad infinitum. So how and why did entering the social TWR niche trigger the evolution of such overwhelmingly eusocial cognition in the hominin mindset?

Riding on the back of the above five considerations I present the following scenario:

(1) To be obsessed with the self, and to mentally attribute that obsession to other creatures, a species must possess an awareness of self and other selves as mentalizing intentional beings in the first place. Track-ways are inherently intentional.

(2) No other ape lineage (or any other species) has ever exploited conspecific trackways (forget those of other species for now), that is, used them as recognizable social markers. Bipedal hominin track-ways are easily recognizable. One's own past trackways must of course have been recognizable by one's self as well.

(3) Therefore the uniquely hominin autobiographical awareness of an intentional self could have evolved through entering the unique cognitive/behavioural niche of exploiting conspecific track-ways as social markers; that is, using TWR for communication and navigation in the social domain, much as dogs use their own and each other's scent-trails and scent-posts: to find their way around in their territories and to ascertain each other's movements and whereabouts.

(4) Social reading of the track-ways of one's self and intimate others created a society that was far more cognitively complex to live in than any other great-ape society because TWR demands a unique narrative form of cognition (as compared to the natural signs all other animals including chimps use for social navigation: calling, visual searching, and scent-trails). A society is obviously more complex if the agents in it are aware of themselves as intentional agents travelling through time. 
(5) There was selection for this unique form of narrative social cognition because of a cognitive arms-race for better TWR skills in the social domain. The narrative faculty incrementally became genetically and structurally entrenched in the pre-hominin mind/brain.

(6) The next major impetus in hominin cognitive evolution came from the environmentally-forced habitual application of this unique social cognitive faculty to tracking other animals in the extractive foraging domain (Shepard's insightful story). It enabled extremely successful predictive reading of animal behaviours; plus highly effective co-operative searching/hunting techniques using TWR plus very simple technology (stones, digging sticks as clubs, bare hands).

(7) So what was initially mainly a social cognitive faculty became a huge "fuel for success"(Godfrey-Smith 1996) when applied in the extractive-foraging domain. Selection for rapid encephalization began, in order to provide more developmentally plastic neural tissue needed to store knowledge gained from the narrative faculty, and the result was the first true hominins by 2 mya.

(8) Therefore the functioning of all co-evolutionary neural/cognitive and cultural developments selected for after the advent of Oldowan Culture still revolved around the incremental evolution of social competences conferred by the narrative faculty.

(9) At the core of the narrative faculty is a cognitive sense of oneself, and therefore others, as intentional agents travelling from the past into the future in what is essentially a narrative manner. (I repeat: Tulving 1985 coined the term autonoesis for this sense of self-knowingness)

(10) Therefore the core sociological and psychological expression of the modern hominin mind/brain is the autonoetic, autobiographical self-narrative, which begins to develop between the age of 3 and 4 in all normal humans. Without developing and maintaining an up-to-date, full functioning self-narrative, maturing humans cannot possibly cope with the complex cultures they are born into (as proved by the pathologies of amnesia and Alzheimer's Disease).

If we accept the above model of our cognitive evolution, no wonder the human primate is so "obsessed with self and society": because our earliest hominin ancestors entered the TWR cognitive niche in the social domain. 
The HNF was and is predominately a faculty for the social cognition that constitutes human folk psychology. We are consummate mind-readers, and those readings have a narrative structure (just think of gossip). To reiterate: neuroscience has now proved conclusively that social narrative mentalizing, in the form of the imagined self interacting with other entities in past and future contexts in the "theatre of the mind" is the default state of the human brain (Addis, Wong et al. 2007).

These imagined entities are in the main fellow humans, but as Shepard (Shepard 1996) goes on to discuss in depth (in a very Jungian psycho-anthropological manner) they can be totem animals, fictional characters, and deities. In this paper I will not discuss these behaviourally modern human cultural and psychological attributes. We are mainly interested in the evolution of the narrative faculty and the four cornerstone competences that made such elsewhere-and-when cognition possible for the mind of Homo sapiens.

\subsection{Summary and Outline of Following Chapters}

The natural signs we call track-ways are vastly different from the signs all other animals use, because they are directional, durable, and combinatory. Therefore they can be highly decoupled from their referents in space and time, and combinations of them can tell a discrete infinity of narratives, like the hominin conventional sign systems we call languages. Only hominins have ever cognitively exploited them. That is why I argue that entering the unique cognitive/behavioural niche of TWR resulted in the cognitive capacities needed to invent all hominin culture and communication.

My second chapter is all about the demands of TWR cognition when used for extractive foraging. It begins with a brief TWR lesson; then (a) exemplifies how much more informational track-ways are than scent trails, and how hunters apply that information; (b) provides a calibration for different levels of TWR skill; (c) describes how early habilenes may have 'thought' when beginning to hunt large mammals; and finally (d) discusses early forms of communication for transmitting TWR information and hunting plans to younger generations.

In chapter three I argue the earliest Oldowans were hunting large mammals (and were not, at first, obligate scavengers) using simple TWR. I then present some highly successful ways of exploiting track-ways information for capturing both large and small animals using the simple technology early hominins had access to. The last section discusses why no other theorists have seen the possible relevance of TWR to our cognitive and cultural evolution. 
These two chapters therefore concentrate on extractive foraging TWR and its application to Oldowan hunting methods. My readers will then understand why the pre-hominin social TWR cognitive niche caused more social complexity than in any other ape society.

In chapter four we turn back in time to our ancestors' initial entry into the TWR niche. I elaborate on the cognitive arms race triggered by entering the social TWR niche after (a) presenting incontrovertible evidence that our forbears were reading conspecific track-ways to some degree before 3.6 million years ago; and (b) explaining why only our ancestors entered this cognitive niche. Presenting the social and psychological details of this cognitive arms race will bring us to just how the hominin narrative faculty (HNF) evolved through social track-ways exploitation. I argue universal great-ape capacities for here-and-now exact imitation and intentionality-reading made track-ways exploitation possible in the first place.

In chapter five I illustrate my models of the HNF and the four cornerstone hominin social competences and very briefly explain how they work with regard to language in particular. I then present developmental evidence that the HNF and these competences begin to come 'on line' between the age of three and four in all normal humans. The chapter ends with a discussion of how the cornerstone elsewhere-and-when social competences differ markedly from the here-and-now competences of apes and human toddlers under the age of three.

In my sixth and final chapter I present my story of the enforced application of the HNF to the interpretation of other animals' track-ways and all other natural signs, as well as planned cultural solutions to environmental contingencies. I argue environmental forcing due to destruction of wetland resources had a large part to play in the appearance of Oldowan Culture after 2.8 mya. Therefore I describe catastrophic environmental events that occurred between 2.8 and 1.8 mya in some detail, and explain how iterated destruction of wetland resources triggered some hominin populations into habitual tracking and carving of large mammals.

I then discuss certain features of Oldowan and Acheulian Cultures, and present a chronological chart of the major developments in our evolutionary trajectory from 4.5 mya to 100ky. A brief comparison with a similar chart (presented recently by Foley and Gamble 2010) helps to explain how my narrative differs from most other versions of our evolution: I place hunting of large mammals much earlier than many other theorists. 
So I am an 'early rich culture, late language' theorist, which is somewhat unorthodox, since most other theorists are either 'early rich culture, early language' or 'still-chimpish early culture, late language' advocates. I will make two broad observations here in initial support of my model: (1) the recent discovery of 3.4 mya cut-marked bones (McPherron et al 2010) very robustly supports my 'early rich culture as compared to apes' evolutionary scenario; and (2) mimesis is a very simple but highly efficient way of telling simple stories about events that happened or may happen in the elsewhere-and-when, demanding no more than the 'acting-as-if' or 'make-believe' role-taking of children's pretend play.

Like Merlin Donald (Donald 1991) I hold that Oldowan Culture was transmitted by mimetic 'showing how' and 'pretending that' communication. For an example, let's use my cougar story. All an Oldowan juvenile hominin returning to his band from a similar close encounter with a leopard would have had to do 'back at camp' was mimic it's scary demeanour by using his fingers in his mouth for pretend fangs and making a roaring noise, and point upriver to get his story across to his fellow hominins ${ }^{13}$.

Before I present my conclusion to the thesis as a whole at the end of chapter six, I briefly explore some remarkable similarities between the natural system of signs we call track-ways and the conventional systems of symbolic signs we call 'natural languages'. I then touch on how these similarities can provide solutions to one or two long-standing problem in the evolution of language debate. I also minimally explore some aspects of the massive effect the emergence of linguistic communication must have had on the hominin narrative mind, re the establishment of what Merlin Donald called mythic culture.

On that note, before beginning my second chapter by giving my readers a brief lesson in track-ways reading, I will end this chapter by stating the perfectly obvious: the natural system of narratively arranged track-ways signs has always been out there in the real world 'asking' to be cognitively exploited by some intelligent terrestrial species. The culturally arbitrary, invented signs of narratively constructed human languages have not.

My 'triggering track-ways theory' is based on the thoroughly common sense notion that we could only have gained the narrative cognition underlying accumulated hominin culture and

\footnotetext{
${ }^{13}$ Perhaps a team of adult males would have then collected a barrage of stones and headed up-river to chase the big cat out of their territory. After all, chimps have been known to opportunistically mob-attack a female leopard in her den and kill her cubs.
} 
communication by learning how to read and communicate to others the real stories told by track-ways, not the other way around. 


\section{Chapter 2: The Nature of Track-ways Cognition}

\subsection{A Quick Lesson in Track-ways Reading (TWR).}

Below is a good depiction of a simple example of track-ways reading (from Lockley 1999).

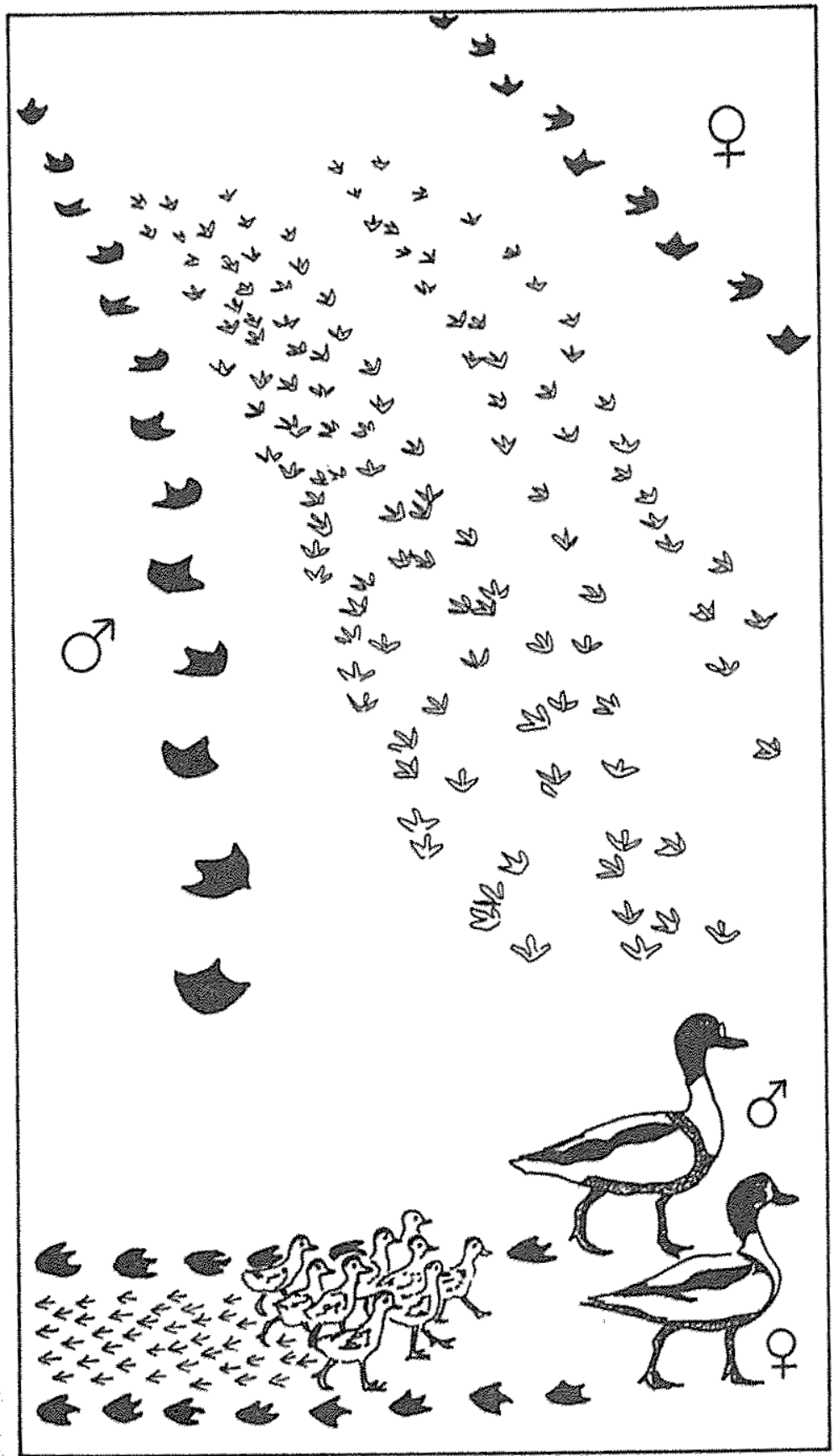

FIGURE 6.5 Tracks of a family of shelducks reveal that protective parents shepherded their ducklings across an open beach. Similar behavior has been suggested for dinosaurs, but without corroborating trackway evidence. 


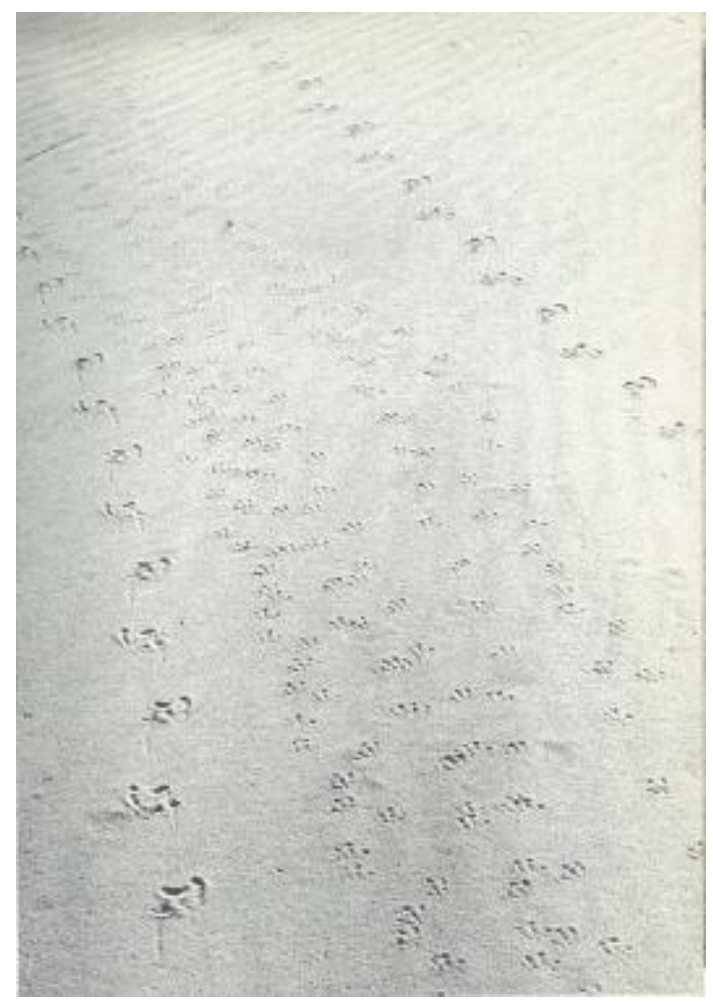

I have added this photo of the real shelduck tracks in case my readers might think the graphic version was unrealistically simple [from (Ennion and Tinbergen 1967), p 23].

The very first sighting of the combination of shelduck footprints in the sand or mud of a beach depicted in the top two-thirds of Lockley's illustration would instantly bring to the mind of any reasonably skilled human tracker a version of the sort of image depicted in the bottom third. This mental representation (image-ination if you like ${ }^{14}$ ) would consist of a pair of shelducks (the largest prints show the male was on the left and the slightly smaller prints indicate its female partner on the right) shepherding their young between them across that beach to the water.

Since all narratives have a beginning and an end, and track-ways are immediately directional, the next image-ination would probably be the end of this shelduck family's journey from land to water. The simplest and most likely ending would be a 'vision' of the family of ducks happily feeding out in the marshes somewhere off shore. Then the beginning of the narrative would automatically come to mind: a mental picture of their nesting site in the low brush

\footnotetext{
${ }^{14}$ I have introduced image-ination here because I think it is important to realize that the trapping and hunting mind (as well as the minds of stone knappers, and all other craftsmen) sees elsewhere-and-when narratives or agent/action scenarios and their possible outcomes in mental pictures, as we do when 'day-dreaming'.
} 
lining the sand dunes behind the beach somewhere, based on having seen the tracks of the ducklings and memories of shelduck nests found in the past. Full-blown TWR involves integration of multiple levels of information, from multiple sensory channels.

With regard to the temporal lineaments of our shelduck 'travelers tale', if they were made on the shore of a sea-side estuary or lagoon, the tracker would know that they had left their nest sometime between the time of sighting the track-way and the last high tide. A far more finegrained temporal indication, or degree of 'freshness', could be ascertained by noting how much water had seeped into those footprint indentations nearest the water. If little or no water has seeped into them from the surrounding very damp substrate they are very fresh indeed.

Such a temporal indication would change the tracker's imagined narrative. The ducks might be hiding in the shore-line swamp reeds just a few feet away because they sensed the tracker coming somehow before she saw them. So it could be worth wading out to have a look if the tracker is a hungry early hominin hunter-gatherer (or a modern wild-life photographer with her camera). In order to find them the tracker would take note of the angle of their last footprints relative to the water's edge, and wade as quietly as possible in that direction whilst crouching down in the reeds of the marsh.

What might be more likely is that a tracker with any knowledge of shelduck behavior would imagine the shelduck family returning to their nest at night-fall. She would then back-track their trail to the nest, look for a good spot to hide near-by, and imagine waiting silently in ambush a bit before night-fall. Inhibiting one's self from heading after the ducks straight away in order to mentally simulate a means/end plan of image-inations of one's self acting in the future, based on memories of one's self usually failing to catch or even get close to ducks by wading in the water, or even to sight them at all in the reeds in the past, is far more likely to have a successful out-come for the hungry hominin or the photographer ${ }^{15}$.

As far as our narrative predictions of differing out-comes are concerned, the photographer will probably be hiding near the nest that very evening because she has imagined getting photos or film-footage of a family of shelducks returning to their nest in the 'magic hour' of evening light in the wild marshes. An inner image-ination of extremely cute ducklings would

\footnotetext{
${ }^{15}$ Especially in N.E. African waters: likely enough a crocodile or two will be lurking in the reeds. Recent evidence however indicates crocodiles were regularly being eaten by hominins at 2 mya. Crocodiles tend to be inactive during the hottest parts of the day, and dislike alluvial highland watersheds, but they would still have been a continual worry for very early hominins.
} 
be the guiding motif of her various mental representations re her inner narrative of possibly selling said photo to some wildlife magazine at some point in the future.

The hominin tracker will probably not be waiting by the nest that evening. Being a mature HG female trying to feed her children (say), she has a different narrative in mind: returning to that same spot in the future with some fellow band-members to capture the young ducks when they are much bigger and fatter but still young enough to be tender. She imagines arriving back with her mates a few weeks in the future when the young ducks are 'flappers' (practicing but still cannot fly) so they will be easy to capture. On the basis of that kind of mental simulation, she may well plant a tall stick in the ground near the nest to make it easier to find when she returns, for from her past experience she knows one bit of the beach by the lagoon looks very much like all the other bits. The stick is a marker that will reduce the load on her cognitive memory when she returns in the future ${ }^{16}$.

These larger, recursive narrative simulations or pictorial 'thought experiments' could have been triggered in the minds of our two imagined trackers by the smaller narrative of the duck's journey from nest to water, which has been triggered in turn by the mere sighting of the trail of prints left in the sand or mud of a beach by the feet of a family of shelducks.

The mental representations in our minds at present have been triggered by my image-inative narrative analysis of the illustration at the beginning of this section, expressed by the letters I have just typed out. These conventional signs are arbitrary symbols that are put together according to a co-operatively agreed-upon system of recursive rules (syntax or grammar) needed in order to transmit an image-inative story from one narrative hominin mind to another using orthographic representations of linguistic communication.

But if we ask ourselves what we are doing when translating these letters into the narrative I have just presented, I think it is fair to say that we are taking turns imagining our selves being in the minds-and-bodies of the ancient hominin, the ducks, and the modern wildlife photographer, at some beach beside a marsh or lagoon we once visited in our personal pasts. That is how the hominin narrative faculty works, that is how the human mind mostly works, and it starts to work like that between the ages of three and four (in all normal humans) when we begin to pretend-play with peers.

\footnotetext{
${ }^{16}$ I think this kind of depictive trail-marking might have begun quite early in hominin evolution. It was an important forerunner to invention of all arbitrary or conventional signs, including the printed words we are using here.
} 
The crux of my thesis is that the kind of cognition needed for reading the narratives told by track-ways is fundamentally a precursor to the cognition needed to understand the words or 'thought-prints' on this page. I argue only the hominin mind possesses this kind of narratively constructed cognition because only our lineage has ever learned to read the narratives told by track-ways. In the next section we shall begin to discover why scent-trails, the signs that all other terrestrial species use for finding preferred resources when foraging, could never have triggered the evolution of narrative cognition. In the process of this discovery my readers will learn much more about how TWR information is exploited when hunting and gathering.

\subsection{Scent-Trailing and TWR: The Cognitive Information Gap}

I will continue to use our shelduck family track-ways narrative, but look at it from the point of view of a scent-trailing dog. We will discover that the amount of information scent-trailing makes available to a dog is extremely limited compared to the amount available to a tracking hominin. There are two physical pathways for scent to be transmitted to the olfactory organs of scenting animals: scent trails deposited on the ground or vegetation above it by bodily contact, and air-borne scents. The hunter's own air-borne scent places a very important constraint on tracking and hunting techniques. All applications of TWR information gained by a hunting hominin tracker have to be geared to the fact that all other mammals are much better at smelling us than we are at smelling them.

Let's return to the point in our tracking story when the tracker has realized the ducks are probably hiding in the reeds very close to the shore-line because very little water has seeped into the ducks' footprints nearest the water's edge. If the ducks are very close a dog that had followed their scent-trail to the water's edge might have the advantage here if there was an onshore breeze. Then the ducks' air-borne scent could pinpoint their position. But only if the breeze was gentle: air-borne scents become highly non-directional in shifting and blustery wind conditions. If the breeze is off-shore a dog would have no idea at all where the ducks were hiding. The tracking hominin would have two advantages over the dog in that case: a greater visual range because of her bipedal posture, and a directional advantage because of the angle of the last tracks seen relative to the water's edge.

If they had waded through some very wide and shallow puddles of water on the way, their scent trail could have already been completely lost by a scenting dog, especially since water would have washed the scent off their feet. For a human tracker the adults' tracks may have still left indentations on the bottom of shallow puddles and would continue to be readable on 
the other side. So we can begin to see immediately in this very simple example how TWR has some advantages over scent-trailing for finding the whereabouts of unseen target animals.

The amount of information potentially available to the scent-trailing dog when compared to the amount available to a track-reading hominin would be highly limited by the physical characteristics of scent:

1) As I pointed out in section 1.2 scent-trails are not combinatorial because any stronger scent overlying, or in close juxtaposition to, a weaker scent makes that weaker scent imperceptible. Track-ways do not have this limitation: several trackways of different animals can over-lie one another and still be discernible. So the dog's brain would probably only register 'duck!' or possibly 'two ducks!' because the young would smell like their parents anyway. The dog could not know there were young ducks with the adults that would be very easy to capture because they could not fly yet. The tracking hominin would have been immediately aware of this possibility, but could also know the young ducks are not worth capturing: the size of their tracks shows they are too young to provide a satisfying meal.

2) The dog might turn and follow the scent-trail backwards for a short distance from where it had disappeared at the water's edge but as soon as the scent got noticeably weaker it would desist. It would then run up and down the water's edge looking for fresher and therefore stronger scent trails before either giving up and moving on or rushing out into the reeds at top speed. It would do the latter if the scent were very fresh and strong; in its associative excitement the dog would be unable to restrain itself.

3) It could well have started following the trail the wrong way in the first place, only switching to the right direction when the scent got weaker towards the dunes. Unlike track-ways scent trails are not immediately directional. Therefore associative here-and-now scent-trailing in general always tends to consume more energy than TWR. The upshot is only the hominin tracker possesses the decoupled narrative cognitive capacity needed to instigate back-tracking to the nest (and she probably would do this as a matter of course). ${ }^{17}$

Since a duck's feet are wet when returning to the nest at night-fall they leave no scent for nocturnal beach-ranging carnivores to follow to the nest. So their nests are hard to find for any nocturnal predator but easily found by a diurnal tracking hominin no matter what time of day it is. I am imagining early hominins with incipient TWR skills foraging for highly nutritious, easily obtainable eggs here. 
4) If it was raining and/or a storm wind was blowing the hominin tracker would still be able to see the prints and hence have a chance of finding the ducks or the nest. For the dog there would be no duck-scent to register at all. Scent trails are quickly obliterated by such conditions, especially in such open environments. Even morning dew in the stillness of dawn is enough to make nocturnally laid-down scent trails in open areas imperceptible. In severe conditions of course, track-ways are also soon obliterated, but in a way the tracker gains another advantage: any track-ways still able to be discerned are sure to be 'dead fresh' and therefore well worth following.

So scent-trails are very ephemeral and short-lived compared to track-ways. This is why dogs so obsessively urinate on certain telephone poles. They are scent-posts for marking out home territory and for communication with other dogs: they are social markers.

To sum this material up, if a scent trail can be perceived, it is always fresh and worth following. There is no incentive for temporal cognition. Track-ways are much more durable, therefore a tracker can be faced with many different track-ways of the same animal, and the choosing of which one is worth following always requires ascertaining when they were made. Thinking about time is the base-line cognitive demand of TWR, which takes the tracking mind away from here-and-now cognition.

Most carnivores do not bother to hunt in the rain or very windy conditions because a) scenttrails are quickly wiped out by water and wind; b) air-borne scents are dispersed by rain and highly non-directional in strong blustery winds. Tracking hominins have even more of an advantage when hunting in such conditions because 1) their own scent is harder for all other scenting animals to perceive, and 2) such conditions make it a lot easier to tell the difference between older and newer footprints. I have sometimes stumbled on to target animals by chance in such conditions simply because they could not smell me due to wind and rain or hear me over the noise of the storm.

One advantage scent-trails do have over track-ways is they can be sensed at night, so the earliest hominin trackers had to have been diurnal foragers in the first place. But hunters with torches can be extremely successful hunting with dogs at night. In general the combination of 
scenting dogs and human trackers is unbeatable when foraging for certain species, but such symbiotic behavior is a behaviourally modern sapiens phenomenon.

Air-borne scents put constraints on both scent-trailing and track-reading (if one is engaged in hunting the author of the tracks at the time). Scenting or tracking downwind is not a good option for predators of course unless they can move a lot faster than their target animal, for their own air-borne scent will precede them. Dogs can move much faster over short distances than humans. This is why human hunters and dogs are such a successful combination when hunting species that tend to bail up and face the dogs. Wind direction does not matter, and the slower human can hone in on the barking of the dogs, run to the scene and dispatch the target animal. However, hunting with dogs on relatively windless days is far more likely to have a successful outcome because wind disperses scent very quickly.

So following ground-scent or track-ways up-wind is the best option; scenting animals further up the trail cannot detect predators until they can see or hear them. However, up-wind airborne scents are only useful if their perpetrator is very close by, due to the vagaries of aircurrents, and only recently laid down scent-trails, even in fairly calm conditions, can be useful to scenting animals. Track-ways can easily last for days and weeks (and millions of years if they become fossilized). Old track-ways very often lead to fresher prints; if a dog does not find a scent trail it has no option but to travel 'blind' until it finds one. This is another reason why dogs and humans make good hunting teams, for the tracking human can lead dogs to scent trails fresh enough for them to be able to follow.

Many animals have far better hearing than humans ${ }^{18}$; this offsets some of the disadvantages of scent perception (consider rabbits, for example). Hence a hominin tracker must learn to move more and more silently as footprints get fresher ${ }^{19}$, as well as be aware of any sudden changes in wind direction. Calibrations of how far the animal is ahead are based on degree of water-seepage in damp ground, relative warmth of feces, sharpness of out-line if in sand or snow, how bent-over grass still is, and so on. A 'gestalt' of tell-tale clues as to freshness builds up over years of experience, until deciding which track-ways to follow is automatized. These fine-grained physical cues are hard to describe in words, for type of substrate and climatic conditions on the day need to be taken into account as well.

\footnotetext{
${ }^{18}$ Hunters in deep closed jungles often use audile perception as much as tracking; our hearing is very directional and phonologically acute, and therefore a very effective adjunct to our excellent vision. Mimicry of a species calls is often used to attract prey into close proximity. This could be important re language capability.

${ }^{19}$ Dogs are a nuisance when hunting many species then, because they do not stalk silently or wait in ambush like many feline species; they depend on team-work and speed to capture prey.
} 
If the spaces between fresh footprints suddenly get much larger a tracker can know the animal has probably detected him and is now running away at top speed. In this case the target animal may not be worth pursuing any further. A dog following a scent-trail cannot detect this kind of information, but then a dog can travel faster than a human, so may still have a chance of catching its prey. Many prey species tend to circle when pursued for they do not like entering unknown territory. Hence dogs can be useful for chasing animals back towards the waiting hunter, but this is a very modern hit-and-miss hunting method, for the target animal will be travelling at speed and only rifles or powerful bows can bring them down (and only if the hunter's aim is very accurate).

If a track-way begins to steadily turn down-wind, a hominin hunter can leave it and backtrack down-wind parallel to where he narratively imagines the animal that made the trackway is heading, then cut across to that imagined route hoping to come onto the animal directly across-wind. If he does not come upon the animal immediately and sees none of its tracks, he can know he is still downwind of the approaching animal, and simply wait in the best place for an ambush. This is only worth doing if the track-way indicated their author or agent was still cruising along or in a stop-start relaxed foraging mode.

The elsewhere-and-when narrative imagination needed for hunting applications based on TWR information entails taking Daniel Dennett's intentional stance. It is very predictively and practically useful to think of the target creature as an intentional agent. A dog of course cannot do this for it has no narrative faculty: leaving the scent trail can only instigate a loss of associative here-and-now cognitive awareness of the target animal's very existence.

Much of the human/dog difference is not about the difference between TWR and scenttrailing; skilful TWR depends on our capacity to integrate path-way perception with other information about the animal, using narrative social mind-reading cognition. ${ }^{20}$ Dogs differ from humans in two ways. Their pathway perception is informationally impoverished compared to humans, and they cannot integrate pathway information with other information.

Obviously a good knowledge of the surrounding territory and the normal behavioural routines of target species are of great help with such hominin hunting methods. Therefore reading the track-ways of all preferred target animals for some time whenever one comes upon them, not just when hunting them, is a very useful practice. For the narratives told by those track-ways

\footnotetext{
${ }^{20}$ This is the basic reason I argue TWR began in the social/navigation domain, not the hunting domain: the narrative faculty is necessary for intentional or goal-directed tracking.
} 
can teach one about their author's habitual routes of travel, what they have been foraging on lately and where.

All this information can be gained without ever seeing target animals until the moment of capture in the distant future. So if TWR has become important in a hominin's foraging behavior, this will select for other cognitive capacities: mental maps of territory in all weathers and time of day based on narrative memory of previous hunting episodes; enlarged semantic memory for natural history information; conditional means/end reasoning, and so on.

The track-ways of all larger animals become recognizable quite quickly for good trackers, for individuals of the same species have slightly different gaits as well as idiosyncratically asymmetrical sized and shaped feet. A kind of informational 'gestalt' of size and shape of track-ways plus other traces such as droppings, angles of damp patches of urination relative to hind-foot prints can often delineate age and gender. Just the way the animal walks, revealed by spatial analysis of its track-way, can tell you whether it is in good condition, sick and old, or wounded and near exhaustion (amount of blood seen, evidence of staggering).

Signs of foraging such as species and height of browsed flora can reveal slightly different food preferences, and so on. One can get to know preferred individual target animals' behavioural idiosyncrasies by following their track-ways whenever one comes across them, not just by direct observation, and observing secretive animals is very difficult ${ }^{21}$. This kind of decoupled or else-where-and-when knowledge is not possible to gain without having the cognitive narrative faculty to read the stories that individual track-ways reveal about a specific animal's character and life-style. Such knowledge would also have made it easy to dodge areas known to be frequented by dangerous predators at certain times of the day or year (remember my cougar story: I avoided that spruce grove for the rest of the winter).

When I was young I once got to know a young mule deer very well indeed by continually reading his track-ways. His territory was also my territory because it was my favourite grouse-hunting and rabbit-snaring area. There was also a huge wild blueberry patch in the centre of it, so I went there almost daily in berry season. Consequently I knew where he could be found at any time of the day, and where he slept at night. Whenever I went fishing I kept an eye out for all tracks of mink in the mud and sand of river beaches. I learned their

\footnotetext{
${ }^{21}$ Hence conservation rangers in cold climates use winter track-ways of many species to estimate population levels. It is a lot easier than trying to observe them.
} 
whereabouts and daily routines through TWR, even though I never got more than a brief glimpse of a single specimen. Therefore I knew where to set my traps for them when winter came and their skins were worth taking.

I have recounted this material because it is by continually reading track-ways that juvenile HGs become very good 'folk-biologists' and hone their TWR skills. It takes many years of such dedicated learning to become a really successful hunter. Consequently only a few of the males in a band may end up supplying the bulk of the meat to that band (Silberbauer 1981; Kaplan, Hill et al. 1984; Kaplan and Hill 1985). We will revisit this important consideration at the end of the next section.

Notice that we have come a long way from our shelduck footprints here. Not just footprints, but a large raft of physically visible traces of travel, are salient to full-blown TWR. TWR can expand incrementally, over ontogeny and phylogeny, from highly visible and distinctive footprints to traces that are either less physically obvious, or more heavily dependent on background information, or both. In the next section we will discuss calibrations of TWR skills. This will help us to estimate the levels of cognitive capacity for TWR possessed by hominins at different stages in their evolution.

\subsection{The Calibration of TWR Cognition: Simple, Systematic, Speculative,}

Liebenberg (1990) described three levels of TWR skill, beginning with very simple, even though he speculated that even simple TWR could not have occurred until quite recently in our evolutionary past ${ }^{22}$ :

1) Simple TWR: the more opportunistic exploitation of track-ways left behind by terrestrial preferred target species (such as other pre-hominins) in easily read substrates such as damp sand, mud, dust, and snow (plus volcanic ash, as we shall discover). A good example would be the track-ways of the shelducks illustrated at the beginning of this chapter.

However, even at this basal level of TWR skill, the amount of information potentially available with any increase in cognitive capacity is very open-ended, as my treatment of the shelduck family's track-way revealed.

\footnotetext{
${ }^{22}$ I believe he has recently adjusted this chronological view towards mine (Lieberberg 2008).
} 
At the beginning of chapter four I present extremely robust evidence that the very simplest level of TWR cognitive demand began to be manifested when pre-hominins began routinely stepping in each other's foot-prints (and their own old footprints) for the sake of safety when negotiating dangerous ground, such as swampy environments full of pockets of deep mud and quick-sand. We modern humans still do this without thinking about it when negotiating wilderness trails; we take note of where the last person on the trail has placed his feet. If we can see that they slipped or got one boot bogged in the mud we try stepping somewhere else, if not we usually imitate them by stepping in their old prints, or very near them.

Dangerous local predator's footprints may have been the first prints of other animals to become salient, given that Ardipithecus ramidus brains were still ape-sized. I posit the desire to know what other conspecifics were up to and where they were (especially one's mother when young or vice versa) would have been more of an 'every day in every way' motivation for pre-hominins to begin reading track-ways than hunting other animals, since chimps capture prey adequately without using TWR (or scent trailing).

However, this idea does not negate the use of simple TWR to follow conspecifics' old trackways (and one's own) in order to re-find foraging niches in very changeable and featureless wet-lands, or safely find one's way back to one's band when lost. Using one's own old tracks for safety and orienteering would obviously have been an everyday occurrence. As we shall see in chapter four, this is a very important idea.

We also need to remember that social and ecological domains have an intimate relationship re competition with other pre-hominin groups and individuals for resources: seeing another conspecific's track-way when foraging means someone else might have already acquired the targeted resource. Seeing your own old track-way means you've already been there, so maybe you should go somewhere new (unless it was a good foraging spot of course). My main point here is that simple TWR could have been quite adequate for the above purposes for the first million or so years of hominin evolution.

2)Systematic TWR: dedicated, goal-directed following of a specific track-way through all terrains by also noting bent-over ground-covering foliage, dislodged pebbles and leaf-litter, blood spots if its author was wounded, and so on till one finds the target animal wherever it may be. So there may have been two roughly 
different stages in TWR skill development: (a) using much more subtle, less obvious clues; and (b) using track-ways information as a core part of planned, long-term behavior (rather than opportunistically, 'on the fly').

I think social TWR may have stayed at stage (a) for a relatively long time before stage (b) emerged, because the second stage is not possible without the narrative faculty. The change to (b) would probably have had its first expression in the situation of mothers and allomothers tracking lost or misplaced offspring. This is an example of intentional or planned rather than simple opportunistic TWR.

Systematic tracking demands more ability to imagine an agent (the target animal) traveling through the environment, because some terrains (rocky riverbeds and outcrops for example) are not easy to find footprints in. One has to begin to imagine where the animal might have been heading when easily-read tracks disappear. This requires a good memory for normal animal behaviours and a theory of mind capacity which can imagine what mental state the animal is in- whether it is frightened and therefore heading for its den or a thicket to hide in, whether it is relaxed and therefore ambling about just foraging, and so on. The systematic tracker is integrating natural history and local geography via Dennett's intentional stance.

The better one is at this kind of narrative imagination, the quicker one is going to find the track-way again (and the target animal). This kind of tracking is especially important when looking for a wounded animal, which may have happened often given the simple killing tools of early hominins. After all, one wants to find the weakening or perhaps already dead animal before some other predator or scavenger with a good sense of smell can find it (all carnivores are very strongly attracted to the smell of blood).

A good narrative autobiographical memory for past experiences when hunting becomes vital. For example, remembering which species tended to go down-hill into gullies when one pursued a wounded member of that species, as apart from those which tended to 'head for the hills' to hole up in some rocky out-crop somewhere. No acquisition of systematic tracking skill is possible therefore without possessing the hominin narrative faculty (HNF) to some degree. I argue this level of tracking skill was beginning to be attained by early erectus hominins, who had brains twice the size of chimps.

3)Speculative TWR: following a track-way simply and/or systematically for a while and then heading directly to where one imagines the target animal might be, right now 
or by the time one gets there, given one's knowledge of its routine behaviours and the surrounding terrain.

This style of tracking above all depends on narrative imagination plus semantic knowledge of the local terrain and animal behavior. The target is now an imagined self, not just an agent with various remembered habits. Selection for anthropomorphizing is occurring but the HNF must already be quite well entrenched. Do not forget that I posit this cognitive level of TWR had to have been at least partly reached in the social domain before it could be applied to hunting individual large animals, thus engendering positive feedback selection for a better narrative faculty. For in the social domain seeing other hominins as intentional agents is not anthropomorphizing. I argue selection for social TWR skills selected for the intentional stance, which was then exapted to improve foraging skills, in combination with TWR.

Speculative tracking is a particularly good way to hunt when the wind is not in your favour: that is, when using systematical TWR will take you up-wind of the animal so it can smell you approaching long before you ever get close enough to capture it. A successful outcome is far more likely if you leave the track-way, circle back and sneak up on where you imagine it is going to be from down-wind. Even if the wind is in your favour, going directly to the place you imagine it might be, rather than systematically keeping to its track-way, can save a lot of time and energy. This depends of course on how often you are right, so !Kung San pairs of hunters are often made up of a systematic tracker and a speculative tracker: ongoing theoretical discussions of possible meanings of track-ways evidence are the norm (Liebenberg 1990).

Speculative tracking obviously demands maximum application of the narrative imagination enabled by the HNF. It requires imagining being in-the-mind-and-body of an unseen agent (the target animal) moving to an unseen place to perform a routine behavior in that place. It also requires imagining one's future-self approaching this unseen (but narratively remembered) place from a certain direction so one can approach the animal up-wind. Any increase in TWR skills and means/ends anticipatory cognition for application of the information gained from TWR will create positive feed-back on other cognitive capacities, and vice versa. So from the inception of Oldowan Culture onwards we are seeing selection for fast-track co-evolutionary expansion of TWR/HNF capacity.

One may also be imagining the best concealed approach to use, the approach one knows is there because one's past-self has been in that place before, probably going through roughly 
similar actions. The whole mental process is highly speculative; the animal might suddenly change direction and go somewhere else, or even be attacked by a predator on the way to the imagined rendezvous. Such is the nature of hominin narrative cognition: we are all heuristic gamblers when it comes down to 'how much effort is it going to take, will it still be there when I get there, and will I get to exploit it or not'. Even though we won't find out till we get there, we still go through all the narrative/imaginative processing of evidence from past experiences of the particular situation.

In general, modern HGs use a combination of systematic/speculative tracking, leaning to one or other according to circumstances, their personal inclinations and level of intelligence. In fact Liebenberg (1990) equates modern HG levels of TWR cognitive capacities with those required for 'scientific inductive hypothesizing'. The possible richness of TWR cognition, folk biology, and stalking skills needed for increasingly successful hunting means:

1) Hunting skills take a long time to learn and are a limiting factor in individual and group fitness for large-brained hominins (brain uses $25 \%$ of energy)

2) Plastically neural developmental stages of extended childhood and juvenility would be selected for to learn these crucial skills ${ }^{23}$.

3) Therefore the time and intelligence needed to learn full-blown TWR informationgathering and its hunting applications should lead to this prediction: the hunting prowess of only a portion of the mature hunters in each band should provide the bulk of the protein, which is the high-quality nutrition needed for all bandmembers to be healthy, fully functional individuals.

4) The best hunters should have more wives and children, have more status, be better social leaders ${ }^{24}$, and so on.

All ethnographic evidence from extant HG bands shows that points (1), (3) and (4) are the norm (Kaplan and Hill 1985). Kim Hill, Hillard Kaplan, Michael Gurven and associated theorists have published a lot of material on the co-evolution of extended human childhood, male provisioning, and the highly skilled nature of foraging activities. Their views complement mine nicely (Lee, DeVore et al. 1969; Kaplan and Hill 1985; Hill, Kaplan et al. 1987; Lancaster, Kaplan et al. 2000; Gurven, Hill et al. 2002; Kaplan, Gurven et al. 2010).

\footnotetext{
${ }^{23}$ Here is where I think post-menopausal grandmothers came into our evolutionary story: not for gathering tubers, but for the extra alloparenting needed to counteract the long-term dependency of hominin children.

${ }^{24}$ I mean when it comes to male politics and alliances, here. If not completely egalitarian, many historical HG tribes were very matriarchal, especially if some horticulture was practiced (for example the Iroquois).
} 
William Laughlin (in Lee and De Vore 1969) long ago argued (under the auspices of biggame hunting theory) that selection for encephalization and neural restructuring needed for hunting skills was the driving force behind human evolution. I partly agree, but we need to temper his 'big-game hunting theory' by (1) arguing for a pre-Oldowan high-quality staple diet of wetland flora and fauna and small terrestrial mammals, and (2) positing a much earlier entry into the socio-ecological TWR niche as the selective trigger for the neural restructuring and encephalization that later made Oldowan hunting and carving of large mammals possible.

I should add here that TWR is not just useful for hunting: it is also important for predator avoidance, finding water, easy routes through rough terrain, and gathering (finding tubers by following pig tracks for example). Kung San women are consummate trackers, often better at tracking than many of the men, and some of them join their husbands in the hunt for that reason (Bisiele and Barclay 2001). All TWR information (and information gleaned from narratively reading all other natural signs) gathered during the day by women gathering, juveniles and old men snaring and trapping, as well as mature male hunters, is communicated to the whole band around the fire in the evening. This information is then used to make plans for the next day’s gathering and hunting (Silberbauer 1981, Liebenberg 1990).

\subsection{Mental Applications of TWR Information: How Early Hominin Hunters} 'Thought'.

What I want to explain now is how habilis/erectus (and earlier hominins navigating through, and to and from, the social domain) may have 'thought'. Since I was a professional hunter and trapper for many years, I will attempt to do so by speaking for myself when it comes to what happens in the mind when trapping. I have chosen trapping because the thought required is the ultimate example of narrative mental simulation of possible future outcomes.

When setting a trap for a certain species I am mentally 'seeing' (or image-ining) just where a member of that species is going to be placing its feet or its head in the future, relative to the physical parameters or context of the space in which I am setting the trap and placing the bait (if used) to lure the animal into it. The reason I am setting the trap 'just there' is not just because all the track-ways and other physical traces normally left by the daily physical passage of the targeted animal are there. 
The other reason is because the surrounds will physically constrain the animal to place its feet or its head exactly where I set the trap. If the surrounds are not 'just right', I artfully place a few twigs in the right place to coax the target animal to physically move in the way I want it to. I have to mentally picture the target animal approaching the whole trap site in the future, essentially by imagining myself to be in the body and mind of the target animal. The as-if role taking is especially pertinent when setting baits and lures above or close to the trap; one tries to use a bait that the target animal could hardly refuse, but present it in as natural a way as possible so that animal will not become suspicious of the whole scene and head elsewhere.

The possibilities raised by application of early hominin narrative social skills to the exigencies of extracting foraging techniques is amply illustrated by the art and science of trapping. It gets quite complicated when one is setting a trap on or near a game trail that all animals use. A conscientious trapper will then try to set the trap and present the lure in such a way that it will catch only the species targeted. If one wants the animal's hide and fur, one tries to make sure there are no snags or sharp projections anywhere within reach of its struggles to get out of the trap. It is all about visualizing physical outcomes in the 'mind's eye'. But then, so is building a house, or doing anything practical.

If one is a very good trapper, and is after highly intelligent and cautious scent-trailing animals such as coyotes, lynx and wolves, one will walk in water as much as possible, wear old gloves that have been left out in the open for months, etc. In general the modus operandi of trapping is to try and leave the whole scene looking as natural as possible, with as little human scent left behind as one can manage. To sum up: when setting traps or snares everything is done according to the perceptions and possible mental state of the animal I am imagining I am being while I am setting the trap.

Then another important problem presents itself when one is setting out a 'line' of perhaps fifty traps or snares. How does one find all of them the next day, when they have been purposefully set so as to be as unobtrusive as possible in the environment? One can use one's own footprints from yesterday, but the trap sites are not easy to see. Environments look very different in different weathers and light conditions. And what if it snows or rains very heavily over night? Your footprints from yesterday will have disappeared.

The best thing to do is leave some sort of easily recognizable markers on the path one has followed, and one near each trap-site, of course, to take the load off cognitive memory. But it still has to be as unobtrusive as possible from the target animal's point of view. And finally, 
if one is setting traps where other unscrupulous human competitors may be travelling, then one tries to invent markers that are only 'readable' by one's self and one's closest allies (in case something happens to you in the interim between setting and checking the traps).

I have presented here the whole gamut of narratively imagining being in 'the minds-andbodies' of target animals, unwanted animals, your future searching self, future searching friends, and unscrupulous searching competitors or enemies. In the process one is inventing conventional signs that are constructed so as to only have meaning for one's self and one's intimate others. In this description of the cognition possibly required for trapping and snaring techniques, I believe we have encapsulated the whole story of the evolution of hominin cognition, culture and communication. It is all based on using the narrative faculty for as-if role playing and the reading of all natural signs, and inventing symbolic deictic (pointing out) communicative signs. This example also exemplifies a seamless gradient between the ecological and social cognitive domains.

My readers may protest that this trapping cognition is so sophisticated that only behaviourally modern humans could engage in it. They are probably right: it is a very fancy application of the intentional stance, and even a snare (a running noose) is quite technologically sophisticated ${ }^{25}$. However, consider a pit dug on a game trail: probably the first kind of animal trap ever invented because it is technologically the simplest. One would still want to leave a sign pointing it out for a foraging band member (perhaps returning to home base with a heavy load) so they would not step into it, even though it will be covered over in some way that looks as natural as possible so that target animals will step into it.

Now we will go back to an even simpler, intermediate stage of hunting/trapping cognition. Swampy wetlands are full of natural animal traps: muddy holes that are deep enough for a large animal to get trapped in, especially after a bit of rain. It is quite possible that a band of Oldowan hominins with brains already twice the size of chimp brains would have a network of such sites within their home territory that they checked out on a regular basis, on the chance that some large ungulate had become mired. Open wetlands are very changeable environments due to the effects of rain on water-levels, and very featureless. Leaving marker poles stuck in the ground near these natural traps would take the load off cognitive memory.

\footnotetext{
${ }^{25}$ There is good evidence of snaring and trapping in the early MSA in South Africa: Wadley, L. (2010). "Were snares and traps used in the Middle Stone Age and does it matter? A review and a case study from Sibudu, South Africa." Journal of Human Evolution 58(2): 179-192.
} 
"They alter the environment to make tasks easier, taking the load off memory by marking a trail and by storing reusable items in the same place. Initially difficult skills become more routine, for one form of Tomasello's Ratchet is task simplification. Hominids make aspects of the physical or social world more salient by marking them physically, linguistically, or behaviourally."

[(Sterelny 2003) p.157]

What I am arguing for here is similar albeit simpler mental simulation capacities for habilis/erectus. Narrative imagination based on as-if role playing would have enabled these hominins to pick a good site for miring large animals and then form a team for singling suitable animals out of passing herds and driving them into it. Or hiding beside a game trail until an animal was in just the right position to be pelted with stones and thus re-directed into a nearby mud-trap. Or setting bait out in shallow water at a good ambushing spot for crocodiles and smaller wetland fauna ${ }^{26}$.

Though the cognitive process is not as anticipatory as trapping and snaring techniques, and the technology is extremely basic, the principle of imagining being target animals, other hominins, or one's future self is the same. The kind of cognition I speculate Oldowans probably had was in the same ballpark as the vivid, action-packed day-dreams children begin to have when older than the age of four. If one accepts a bit of 'ontogeny recapitulates phylogeny' here, this idea would seem plausible.

The upshot of what I am getting at here is that Oldowans were already a lot smarter than chimps. The idea of Oldowan Culture suddenly emerging from a chimp-like mind-set because of environmental contingencies does not make sense for a very obvious reason: why didn't other ape lineages respond to those contingencies with something like Oldowan Culture? As Merlin Donald (in Corballis and Lea 1991) points out, such theorizing 'puts the cultural cart before the cognitive horse'.

Narrative reading of all natural and conventional signs is the bottom-line cognitive skill to the HG life-way, and its application to social navigation and foraging requires reality-based narrative mimetic/depictive mental simulations of where, when and what other agents were, are, or will be doing in the else-where-and-when-which includes right now when you

\footnotetext{
${ }^{26}$ I shall return to this idea in chapter three. It can explain some very puzzling features of many Oldowan and Acheulian stone and bone sites.
} 
cannot see or hear them. Perhaps I should delineate here between TWR itself and the narrative faculty needed to use track-way information efficiently.

Much of hunting mental simulation is not TWR, it is using TWR, but spatial analysis of track-ways itself requires mental simulation of the target animal's physical and mental characteristics, as if they were mentally goal-oriented or intentional like us. The elements of TWR and HNF cognition work very much in socio-ecological tandem, and so the neural capacity for using both would have continued to co-evolve through selection for using mindreading skills when tracking and when socializing.

Mental simulation/attribution or theory of mind ${ }^{27}$ has typically been assumed to be a social skill, used in social interaction with other humans. My point here is that it is also, and perhaps uniquely, a hominin foraging skill. San hunters could predict the characters of their target animals when observing a herd of animals: whether they would be easy to stalk (how cautious or stupid), and how dangerous they would be ${ }^{28}$ at the kill (Silberbauer 1981). All this was read as well as their sex, age, condition, and so on. They would thereby choose which individual to hunt. Silberbauer stated they were never wrong.

They could also do this to some extent by analyzing track-ways that recorded interactions between already targeted animals and their cohorts, in the logical manner referred to in my introductory section on TWR cognition. This anthropomorphic reading of animal psychology resonates with, and I would argue is derived from, classic folk psychology in the social domain, engendered by the HNF. This strongly indicates the HNF in turn was initially selected for in the social domain, ever since the original pre-hominins entered the unique TWR cognitive niche for the purposes of navigation.

And last but not least, such mind reading of other animals' perceptive abilities and character when stalking them and setting traps for them very much includes awareness of yourself in the present, as well as in the somewhat remembered past and all the possible futures, for one must learn how to make one's own physical influence on the local environment as minimal as possible. Now we can fully understand why only a few males in any HG band are really good

\footnotetext{
${ }^{27} \mathrm{My}$ view of theory of mind and the intentional stance is very much in the imagination-simulation tradition, as distinct from the 'theory-theory' tradition which sees the intentional stance as semantic knowledge, not imagination. Defending my simulationist view is beyond the scope of this thesis, but basically I hold that an autonoetic awareness of one's own intentionality and mental states is required before one can imagine the possible mental states of another agent.

${ }^{28}$ He said he counted 18 categories they used for describing the characters of individuals of habitually targeted species, but he does not describe them.
} 
hunters, and why they also tend to be the best social communicators and politicians, and to have more wives and more children. Hunting prowess, based on the intricacies of physical and mental application of narrative TWR skills, has been an extremely powerful selective factor in the co-evolution of all hominin cognitive capacities. What must have been equally important was the capacity to pass as much hunting/gathering knowledge and skills as possible on to the next generation.

\subsection{The Pedagogy or Cultural Transmission of TWR Skills}

Since simpler TWR skills could have been taught to ensuing generations by early mimetic hominins using physical communicative as-if role-taking methods resembling pretend play (for example by pointing to or drawing tracks of dangerous predators and miming their scary demeanour) and in fact can only be learnt via similar 'showing how' methods, my view that at least some degree of TWR was a very ancient proto-hominin skill is very plausible indeed.

HG children are taught how to read track-ways of dangerous predators and important food species, by procedural showing and depiction, from the age of three (Morrison 1981). Silberbauer (1981) said San juveniles learn most of their TWR skills from auto-rehearsal and trial-and-error direct experience however, when they start applying "simple" hunting techniques like setting snares, learnt from older peers or the old men of the tribe. As we now know, setting snares is not at all simple.

Like all HG children I started trying to snare and trap small animals after the age of six. I was only occasionally directly taught how to read track-ways 'on the job' as it were, by my father and an old trapper that took an interest in my welfare and gave me half a dozen traps when I was about eight. I also absorbed some knowledge of what to look for by spending many hours listening to hunting stories told by my father and his friends. Such is the efficiency of linguistic communication, but miming would have been far more physically informative, and theatrical miming of hunting stories is the norm around $\mathrm{HG}$ campfires in the evening.

The real learning came from doing, from experience, over and above being shown how. The !Kung San say that it is better for a young hunter to learn to read track-ways on his own just by 'doing' it, for by learning that way "he will know his own mind" (Liebenberg 1990). No matter how they learn it, reading track-ways for all HG children becomes as 'second-nature', or neurally automatized, as comprehending someone else's spoken story. 
The main point here is that TWR cannot be learned or taught by using language, only by learning by doing, being shown how via mimetic/deictic teaching while hunting with older hominins, watching mimed hunting stories around evening fires, and pedagogical depiction of track-ways in suitable substrates. In the case of human track-ways, all !Kung San children know their mother's footprints from all others in the band by the age of four without directed teaching. In fact, trackers can recognize the passage of a known human, from their track-way alone, even though they have not seen that person's tracks for over twenty years. San hunters burst out laughing when Silberbauer (1981) asked them if it was easy to recognize individual human track-ways: that is how silly they thought the question was.

Here is yet another reason I argue TWR began in the social domain: because directed teaching or encouragement is not needed when it comes to taking note of all band-members' track-ways. Perhaps in the beginning only the shape of footprints of dangerous animals was drawn or pointed out for very young proto-hominins that were still too protected to have seen such animals. One gets the impression from the ethnographic literature track-drawing 'lessons' were something historical HGs did for the fun and the challenge of drawing realistic track-ways, as when sitting around after a good meal. Early hominins probably could not do this, but they could point to tracks of different species and mime the animals that made them.

For example, see these ethnographic descriptions of Australian aboriginal traditional teaching behaviours below, followed by some of their remarkably 'life-like' track-drawings on the following page (cited in and borrowed from Morrison 1981):

"Next to the 'catch-cradle'[cats-cradle], the most general perhaps of all imitative games is the drawing of different animal and bird tracks...in the smoothed sand, by means of the fingers, finger-nails, palms, small sticks..."

Walter Roth 1902

"It appears as though the older more knowledgeable members of the tribe would draw tracks of animals in the sand for the young children to identify. Attention was paid to such detail as direction of travel and how much time had elapsed since the passage of the animal". (my emphasis) 


\section{The First Pictographs}

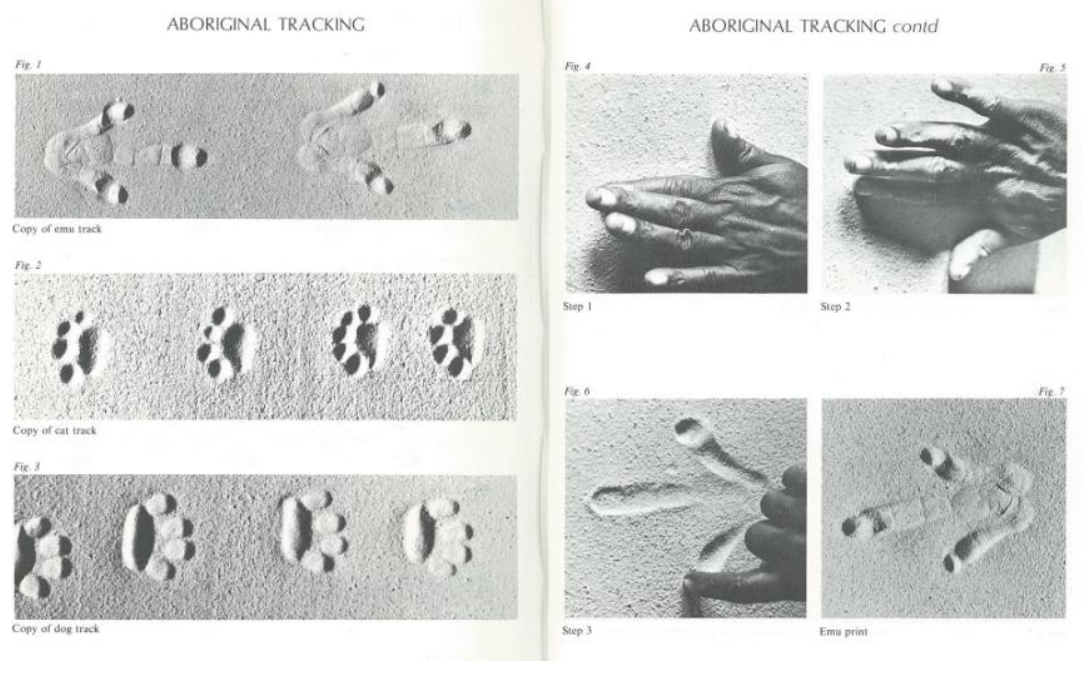

Conventional depictions of combinations of track-ways were also part of Aboriginal art (see below).

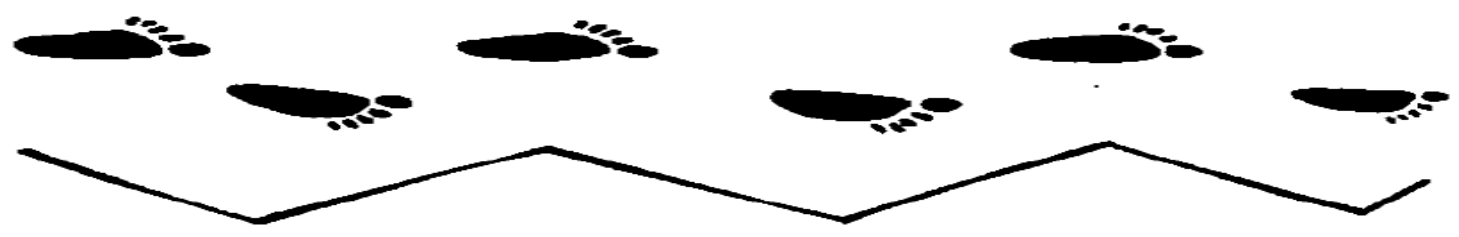

Human footprints and trail

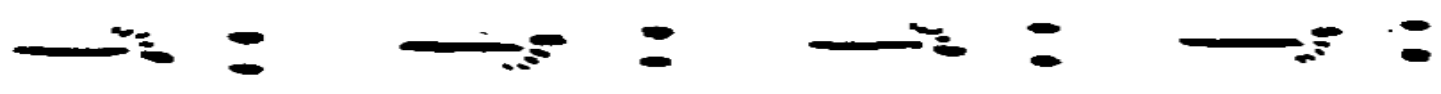

'A man is tracking a rabbit'. Simple example of pictography

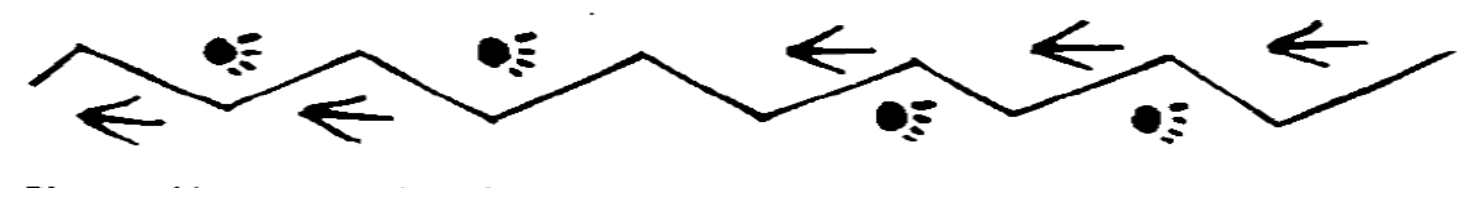

The pictograph immediately above (they are all borrowed from Morrison 1981) depicts a dog following an emu trail, but the dog would be following the emu's scent trail of course. In this 
simple depiction and the top one above, the zig-zagging line is graphically symbolic of the concept of 'trail', a pathway through any environment.

Conveying TWR information and drawing simple directional maps for hunting plans for other early hominin minds was probably the origin of mimetic/depictive signs such as those above, made in or on suitable substrates. Combinations of mimetic/depictive and gestural signs made with the whole body and/or the hands in the air, referring to specific species (see illustration next page, borrowed from Liebenberg 1990) and hunting techniques, would have been other forms of the earliest intentional narrative communication.

I fully concur with Merlin Donald's excellent work (Donald 1991) on how mimetic showinghow communication had to have been in place to transmitting Oldowan Culture between generations. I refer not just to stone- knapping skills. Think of hunting techniques; foot-prints of different species; how to make a simple spear; how to make a throwing club weighted by a large bulge at one end, a simple carrying sling and so on. Oldowan juveniles in turn must have been able to represent action schematas of the self and others in order to imitate older more skilled peers when they were not around. They would have needed such mental schematas to intentionally auto-rehearse such skills through trial-and-error repetition when on their own or with other juveniles (as in pretend play).

What I add to Merlin Donald's argument is that these hierarchical action schemata or sequences were narratively constructed, because they also include in the sequence who is doing what (the action) to whom. This structure equals the propositional agent-action-goal syntax of minimal or thematic grammar. I will not discuss grammar or syntax much further in this paper: the theoretical territory is voluminous, and, I believe, fairly irrelevant to human evolution if one accepts the HNF is the module for all higher human cognitive domains (which are plastically developmental not modular). The particular grammar of the languageculture one is born into, as well as the lexicon, has to be learnt at an early age when the brain is still plastically developmental, just like the most important TWR signs and the folk biology of one's local ecology.

All HG hunters use pantomime or mimesis, gestural signs, depictions and mimicked bird calls for communication in order to maintain silence while hunting. They do the same when acting-out hunting stories around the evening camp fire. Groups of women and older children and toddlers talk and sing continuously when out gathering fruit and nuts and digging up 

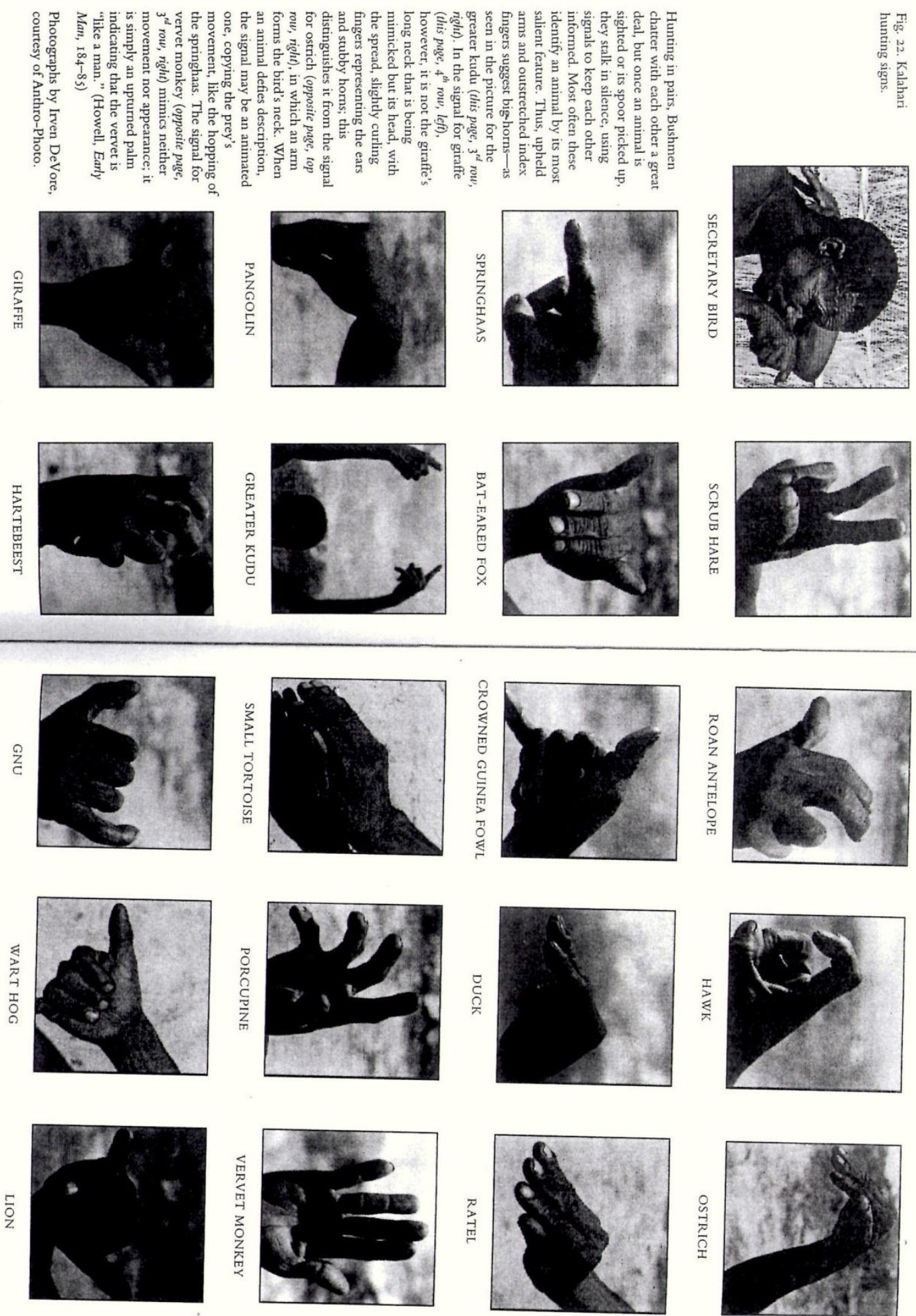
small fauna and tubers (Silberbauer 1981). They do this to deter predators. I posit that prelinguistic vocalizing was restricted to prosodic singing/chanting exchanges (like Gelada baboons continuously vocalizing) and mimicking of other animals and natural sounds when hunting or acting out stories.

The fact that silence is so crucial to hunting success may be one good reason linguistic communication did not begin to emerge in the hominin lineage until the Mid-to-Late Pleistocene, after fire-control became a cultural foundation around .8 mya. Another may be that gestural signs were perfectly adequate for transmitting tracking information, perhaps even more efficient than words ${ }^{29}$. Prior to this emergence, like Michael Corballis (Corballis 1992; Corballis 2002; Corballis 2010) and Armstrong (Armstrong, Stokoe et al. 1995), I think gestural sign-languages had been prevalent since around 1.6 mya. This date heralds the beginning of hand-axe manufacture, the signature of Acheulian Culture. Prosodic ululating or chanting, percussion, dancing and so on probably supplied the social grooming that Robin Dunbar so famously attributes to linguistic gossip.

In sum then re hominin communication: I argue the phonological complexity of hominin mimicry of other animals, each other during group chanting sessions, and noises made by the world around them (Botha and Knight 2009) was co-opted for speech after fire control was invented. Grammar arose out of the narrative construction of the metaphorical/mimetic depictive representations already inculcated in the pre-linguistic hominin mind/brain. The cognitive capacities for language emergence were provided by the HNF, accumulated cooperative mimetic/gestural culture, and the plastically developmental neural capacity conferred by a brain now much more than twice the size of the ape-brain.

This co-evolutionary cultural scaffolding scenario is now not at all unorthodox. What I have added is the evolution of the HNF as the cognitive prerequisite for the cognitive social competences required for all forms of hominin co-operative/intentional culture and communication, triggered by our ancestral entry into the unique TWR cognitive niche.

Learning how to narratively read and apply the track-ways information exhibited by different environments and ecologies has always been neurally plastic, just as it is with learning the

\footnotetext{
${ }^{29}$ The exact speed at which and animal is travelling, as indicated by the gaps between footprints in its trackway, are communicated very accurately from one San tracker to another by a flicking the palm of one hand with the fingers of the other at differing rates (Liebenberg 1990). One cannot verbally describe such fine-grained physical phenomena. It is like trying to describe a spiral with words rather than simply draw it in the air.
} 
language, conventional orthography, the symbolic rituals and behavioural norms of the cultures we moderns are born into. This makes pragmatic sense for a generalist exploratory species because of the differing local ecologies and environmental variability they could encounter. As explained in this chapter (and in introductory section 1.2) there has always been a discrete infinity of track-ways narratives out there in terrestrial environments to be read, just as there is in the symbolic signs of natural languages.

There is no limit to the amount of useful information to be gained from track-ways, especially when it comes to learning the habits of conspecifics, as well as all the animals in one's local ecology. Modern forensic crime investigations still take great heed of any track-ways left by miscreants: blood-stained shoe-prints and foot-prints for example. As I have mentioned before in passing, conservation officers regularly use track-ways to assess population numbers of species in wild-life reserves. The only constraint to gathering TWR information, just as in scientific pursuits, is in the eyes ${ }^{30}$, intelligence, and measuring technology of the investigator. For a very modern example, just recently an article appeared in my local newspaper re new TWR techniques needed to help pest controllers eradicate rats in nature reserves (because they predate on native birds' eggs and fledglings).

The reserve administrators have set up natural-looking false tunnels containing ink-pads and blotting paper to record the footprints of the three different species of marauding rats. The eyes of a human tracker cannot tell them apart, so these track-ways will be analyzed by computer. The reason this is being done is that each rat species has different behavioural traits when it comes to navigation and foraging. Knowing which species they are dealing with will enable pest eradicators to set their traps more appropriately and therefore efficiently if only one rat species is still a problem in the reserve. Their greatest hope is that the computer can tell whether a rat is a pregnant female or not, because concentrating on killing pregnant females is far more efficacious than killing males if you want to eradicate a species from any given area as quickly as possible ${ }^{31}$.

I think hominins were always hunters, and not just because it is crucial to my narrative about the role of TWR in hominin evolution. I argue that co-operative hunting of small wetland

\footnotetext{
${ }^{30}$ From personal experience I think it is very possible that one unassailable reason HG hunters tend to give up hunting in their late forties is due to failing eye-sight. I can buy a pair of glasses at the local chemist; many HGs cannot.

${ }^{31}$ Having once been a professional hunter and trapper of several species of introduced pest animals I know that mature females of any species are much harder to capture than juveniles and mature males, for they are far more cautious. Consequently they are usually the last members of a population to be eradicated.
} 
fauna was very ancient, and some hominin populations began habitual intentional hunting and carving of medium to large mammals after 2.8 mya due to iterated, large-scale destruction of their preferred wetland habitats, not because they suddenly became smart enough to do so. In my model they could suddenly do so because they had been smarter than chimps for a very long time, due to their very early entry into the TWR niche. The new evidence of the cognitive capacity to transport stone tools for $6 \mathrm{kms}$ and carve an animal as large as a cow (McPherron et al 2010) strongly supports this chronological view.

I place intentional tracking and co-operative hunting of large mammals as early as 2.5 mya in our evolutionary journey. This view is at present quite unorthodox (for an orthodox chronology of hunting see Foley and Gamble 2009). If the chronology of my TWR/HNF model is correct, then:

(1) Task analysis of all paleo-archaeological evidence from 2.6 my onwards should indicate the co-operative use of means/ends prospective cognition using narrative mimetic/depictive 'image-ination' and communication, which is not possible without the HNF. For as we have seen, intentional, systematic tracking and hunting techniques are also not possible without narrative, as-if role playing imagination.

(2) Where hard evidence reflecting such cognitive development is not available, then historical and modern HG ethnography should exhibit overwhelming evidence of possible applications of the HNF to the invention and communication of cooperative hunting techniques that require only the kinds of very simple technology that early hominins must have had access to (plus some degree of TWR skills).

(3) The earliest Oldowan endocasts should reflect expansion and restructuring of the core brain areas that support narrative mentalizing and the social competences, and neuro-imaging should show that the human brain is fundamentally a 'narrative machine' due to our very early entry into the TWR niche.

To sum up: the natural signs we call track-ways are vastly different from the signs all other animals use, mainly because they can be so fully decoupled from their referents in space and time, and because combinations of them can tell a discrete infinity of narratives, just like languages. Only hominins have ever cognitively exploited them, and that is why we are the narrative species. This chapter has concentrated on extractive foraging applications, using the $\mathrm{HNF}$, of the information gained from reading the track-ways and associated traces of other 
animals. I have claimed that applying social TWR and the social competences conferred by the HNF to co-operatively tracking, hunting down and carving up large mammals constituted an ecological breakthrough for Oldowan hominins. In the next chapter we discuss what evidence there is for this.

$* * * * * * * * * * * * * * * * * * * * * * * * * * *$ 


\section{Chapter Three: Oldowan Hunters and Gatherers}

\subsection{Big Game Hunters or Obligate Scavengers?}

The claim that narrative reading and application of track-ways information was an ecological breakthrough for Oldowan hominins does not stand or fall on whether they were large game hunters or obligate scavengers. My bottom line is that TWR would have always paid its way through some combination of: (a) using conspecific tracks as navigational aids; (b) predator avoidance; (c) small game hunting; (d) increasing scavenging efficiency. However, in this crucial chapter I present evidence that TWR and the HNF became extremely important soon after 2.8 mya for intentionally obtaining and processing large mammals, and they were not being acquired by obligate passive or confrontational scavenging from other carnivores on a regular basis until near the beginning of Acheulian Culture at 1.6 mya.

While tracking large animals on a habitual basis may not have begun until 2.5 mya, conspecific TWR was important in the social and ecological domains from the very beginning in open featureless wetland environments. I repeat: the social and ecological domains must have an intimate relationship re competition with conspecific groups and individuals for resources: seeing another hominin's track-way when foraging means someone else might have already acquired the targeted resource. As we shall see in chapter four, one's own old track-ways are also extremely important when foraging: for example they reveal that one has already foraged in a certain area. Such basic orienteering is important for optimal resource extraction.

Here are my major reasons for placing hunting behaviours much earlier than most other theorists:

1) Chimps group-hunt animals smaller than themselves and many other primates consume eggs, fledglings, and small invertebrates, so pre-hominins are likely to have done so as well. Only one troupe has ever been seen to scavenge another predator's fresh kill, and only once (a leopard's kill stashed in a tree interestingly enough). Robbing a fresh kill directly from baboons has also been observed, but again, only once or twice.

2) Like all primates we have an innate disgust reaction to the smell of meat that is 'off', unlike dogs and hyenas. 
3) Eating the flesh of small aquatic and terrestrial animals would require little or no carving (or any form of dangerous competition with other carnivores) and therefore would have been the easiest, optimal means of acquiring the high-quality nutrients demanded by proto-hominin encephalization from the very beginning (Broadhurst, Cunnane et al. 1998), (Cunnane and Stewart 2010)

4) Ethnographic studies prove ((Kaplan and Hill 1985),(Stewart 1994) (Gilmore 1953) that simple co-operative hunting techniques using nothing more than bare hands or digging sticks doubling as clubs can provide large quantities of protein from fish, reptiles and small land-based mammals.

5) A perusal of the original literature shows good evidence of fish, reptile and micromammal exploitation was ignored by theorists because of big-game hunting theoretical ideology during the 1970's (Verhaegen and Puech 2010², Cunnane and Stewart 2010) There were thousands of catfish fossils at Beds I and II, Olduvai (Stewart 1994).

6) Recent re-appraisals of hard evidence from some of the earliest stone and bone caches (2.6 to 2 mya) indicates the predominant exploitation of small to medium ungulates (size 2 and $3=26$ to $114 \mathrm{~kg}$ ) as well as some larger size 4 animals, and that hominins were definitely processing them before scavengers and other predators (Plummer 2004; Diez-Martín, Sánchez et al. 2009; Domínguez-Rodrigo, Mabulla et al. 2009).

7) True erectus hominins already had a robust modern human physique and ability to dissipate heat by sweating geared to endurance running during the hottest parts of the day. No other large mammals possess these traits. Modern and historical HGs obtain medium to large game by running them to heat-exhaustion (using TWR to find them when temporarily out of sight) and dispatching them with clubs or spears ((Liebenberg 2008). I will describe this hunting technique in more detail later in this chapter.

\footnotetext{
${ }^{32}$ They quote Leakey et al (1971) re the Olduvai O.H.24 habilis site: 'Crocodile remains predominate among the faunal material from this site and more than 2,000 teeth were found. Tortoise plates, shells of Urocyclid slugs, fish vertebrae and scales, bird bones and pieces of ostrich eggshell were also relatively common'.
} 
8) Scavengers masticate and ingest all remains of smaller animals completely and the bones of small ungulates deteriorate rapidly in the open environment. Unless such remains are very fortuitously almost immediately covered by suitable fossilizing substrates they are much less likely to survive in the archaeological record than the bones of very large mammals. One very early site like this has recently been discovered, dated 1.95 mya: crocodiles, catfish and turtles were being eaten regularly. Another site with large amounts of hominin-derived aquatic fauna fossils is dated 1.5 mya (Braun, Harris et al. 2010).

9) Most small fauna would have been consumed in situ before the advent of fire and cave-shelters as home bases after $1 \mathrm{Ma}$; that is, remains of small fauna would hardly register in the archaeological record at all until the advent of scavenger-free home-based cooking life-ways

10) Most terrestrial tubers are seasonal and inedible (indigestible and/or poisonous) without prolonged processing and cooking, whereas many aquatic corms and rhizomes are sweet, juicy, easily digestible in their raw state (especially if pounded), extremely easy to pull from soft mud, and non-seasonal (for example roots of papyrus and cat-tail sedges which are common fare to this day). The very few theorists supporting the idea of USOs as a staple diet for early hominins (for example (Wrangham and Conklin-Brittain 2003) had to assume that fire was under systematic control as early as 2 mya and there is no evidence for this assumption (Plummer 2004)(Shipman 2009). Exploitation of nuts is a far safer bet before fire and cooking, but nuts are also seasonal.

11) Until quite recently very few theorists have considered the possibility of several species of large fresh-water shell-fish, huge seasonal runs of several species of large, very fatty cat-fish, and small reptiles and mammals being main-stay dietary protein sources in lacustrine/riparian/alluvial areas of the Rift for most of the year (Broadhurst et al 1998, Cunnane and Stewart 2010). Hunting of medium and large mammals, and the opportunistic scavenging of mired, sick or very young animals, probably occurred mostly during the dry season when ungulates were restricted to using infrequent remaining water-holes (Broadhurst et al 1998). 
12) The human tapeworm Taenus solium did not split off phylogenetically from canid, felid and hyaenid tapeworms until 1.7 mya at the earliest ${ }^{33}$ (Shipman 2009). Tapeworms are host-specific and cannot survive in hosts that are not constant meat eaters. This switch would surely have occurred much earlier if hominins had been fueling their encephalization solely by obligate confrontational or passive scavenging from other carnivores since 2.6 mya.

I think theoretical postulations that early hominins were: (a) predominately big-game hunters; (b) obligate scavengers; (c) survived on tubers dug up by post-menopausal grandmothers; (d) only hunted big game after 500ka; (e) only gathered shell-fish, turtles and other small aquatic fauna during the Late Pleistocene, are all seriously flawed. Like a few other theorists (Broadhurst et al 1998 for example), I think wetland fauna and flora was the staple diet of early hominins until massive environmental changes in the Rift after 2.8 mya, but I place obligate scavenging at least a million years later than they do.

\subsection{When and Why Erectus Became Scavengers.}

I posit the human host-specific tapeworm evolved when some populations of erectus hominins became frequent obligate passive and confrontational scavengers of other carnivores' kills after 1.7 mya. This was because they finally had to, due to a combination of these factors: (1) they had become extremely successful, highly competitive, eusocial carnivores; (2) their populations had expanded a great deal; and (3) iterated volcanic and earthquake effects and increasing aridity ever since 2.6 mya had destroyed a large proportion of wetland habitats in the Rift. I describe and present the chronology of some of these environmental events in my final chapter.

Near starvation towards the end of the dry season would have made stealing meat from even the most dangerous predators imperative, and the only other food source that is as high quality 'brain food' as fish and shellfish is marrow and brains. Marrow and brains, obtained by smashing large bones and skulls, would have very often been the only nutrients available from the remnants of large carcasses left behind by lions and other large felids (Schaller and Lowther 1969). Leopard kills stashed in trees would have been a far better choice. TWR skill combined with knowledge of the routines of local leopards could have made these easy to

\footnotetext{
${ }^{33}$ And it could have been as late as .7 mya.
} 
find, but it would still have been a dangerous form of foraging. There were also several species of other huge felids around at the time, such as sabre-tooth tigers.

Like Schaller and Lowther I think Wild African Dog packs would have been the safest carnivores for club-wielding teams of large erectus males to confront and the easiest to find because they are diurnal relay pack-hunters. Hyenas, lions and leopards are mainly nocturnal predators. We know from the Turkana Boy that erectus (not the habilenes: they were probably too small) definitely had the physique of good endurance runners by 1.6 mya. I posit the canid tapeworm transferred to hominins as a specific host because these later hominins had begun to regularly home in on hunting African dog packs (probably by following their tracks). They carved large portions from their fresh kills, and left them the butchering remnants.

In the past there has been a lot of controversy over the evidence, but recent re-analyses of some early sites show quite clearly that hominins from 2.6 mya onwards were regularly gaining access to medium and large mammals before other carnivores (Plummer 2004 for excellent overview). This added to the appearance of the hominin tapeworm a million years later strongly indicates Oldowan hominins could not have begun as obligate scavengers. I think we must return to a far more nuanced version of big-game hunting theories. I bet that teams of habilenes started big-game hunting by using simple TWR to track down large mammals (such as hippos) and startle them by throwing stones so they became mired in deep mud holes.

During many years of trapping and hunting as a pest controller around farms I saw countless cattle and sheep mired in swamps and muddy creeks. Ungulate herds always follow the same trails, so after many animals have used the same crossing of a boggy water-course, the older and weaker animals at the tail end of the herd are very prone to becoming mired in the deep mud created by those that have gone before. They soon become completely exhausted, cannot defend themselves, and would have been easy to dispatch with a club. Bipedal semi-aquatic hominins with some TWR skill would have had an advantage over all other scent-trailing, quadruped predators in such conditions.

Like Rose and Marshall (Rose and Marshall 1996) I also think the 'origin-myth' of timid, non-lethal early hominins cowering together from huge predators needs to be placed in the theoretical waste-bin in favour of a more balanced view. Of course lone foraging hominins and toddlers wandering too far from the band would have been highly vulnerable, and 
everyone would have been somewhat vulnerable at night before fire control. On the other hand, why would predators bother to attack a mob of shouting, stone-throwing, spearing, clubbing proto-hominins who could hurt them from a distance?

Studies have also shown that wetland/riparian environments are remarkably predator free compared to open savannah [(Pickering and Bunn 2007) for example]. I think the reason for this is quite simple: large scenting carnivores do not like hunting in watery environments, and cats in particular do not like getting wet.

Rose and Marshall (and Plummer 2004) argue for defendable home bases that hominins retreated to with large carcass portions. Clark (reply to their 1996 paper) also suggested that carving sites were close to good miring spots, and quotes several instances of historical HGs habitually driving large animals into swamps and water in certain suitable sites in order to immobilize them and kill them.

TWR would always have been salient to hominin life-ways to some degree for safety and socio-ecological navigation to and from home bases or safe refuges. As the narrative faculty evolved, their tracking and co-operative hunting techniques would become more intentional and less opportunistic. Anticipatory cognition would lead to simple co-operative applications of the information gained from their TWR skills, such as finding large mammals and driving them into suitable miring sites.

A recent reworking of one of Leakey's Olduvai sites (Dominguez-Rodrigo et al 2009)(Dominguez-Rodrigo, Bunn et al. 2010) ${ }^{34}$ has shown clearly that small to medium as well as a few very large size 4 animals (Pelorovis) were continually being butchered by hominins before scavengers had access to them. New sites at Lake Turkana have also shown that hippos and giraffes were being carved (Pobiner, Rogers et al. 2008) before scavengers had any access to them. When all this new evidence is added to such evidence of carving plus recent re-evaluations of the anticipatory cognition needed for creating even the earliest Oldowan stone tools (Heinzelin, Clark et al. 1999),(Toth and Schick 2009) it becomes very obvious that Oldowan Culture could not have been created by a creature with a still chimpish mind-set.

\footnotetext{
${ }^{34}$ There was a permanent fresh-water spring $200 \mathrm{~m}$ away. The site was used over many thousands of years, and the make up the faunal age-patterns proves they could not have been the kills of other large predators.
} 
One of the Pelorovis (giant long-horned buffalo, now extinct) specimens appeared to have been mired in a boggy spot right beside the old Leakey site. There are several other sites mentioned in the literature where single very large animals were butchered in situ in muddy places. I think it is time to put the theory of very early obligate scavenging of large mammals to rest.

Why would the earliest Oldowan hominins risk confronting dangerous predators for their kills when they could easily drive large animals into the swamps that were their preferred habitat, and where they had the advantage of bipedality and the free-handed use of stones and clubs? Mired animals exhaust themselves very quickly due to their own efforts to get free, and have no leverage for defensive manoeuvres.

Another very important consideration is that large quadruped predators and scavengers are also at a disadvantage in deep mud. I have already pointed out that large terrestrial carnivores dislike hunting or scavenging in wetlands. There would have been little competition from other terrestrial carnivores in such places. Crocodiles could not have been much of a problem since they were being eaten by hominins at 1.95 mya (Braun et al 2010) and other early sites (Verhagen and Puech 2010). Besides, crocodiles do not need to feed nearly as often as mammalian predators, and are inactive during the hottest part of the day.

\subsection{Erectus Hominins: Endurance Hunters}

The consideration of TWR skills becoming more intentional or systematic as the HNF expanded with encephalization leads us to another important kind of hunting by erectus that many theorists have argued for: endurance hunting (Schaller and Lowther 1969, Bramble and Lieberman 2004, Liebenberg 2008). We know that erectus/ergaster was very robust and fully human sized by 1.6 mya and we know that endurance hunting was habitually practiced by some historical and modern HGs.

Recent studies of the !Kung San by Liebenberg (2008) have shown that endurance hunting is not possible without systematic TWR skills. The faster target animal runs out of sight, seeks shade to recover from heat exhaustion, then is tracked down as quickly as possible by a team of jogging hunters and flushed out from its shady retreat. A specific animal's track-way has to be followed through all types of terrain for this kind of hunting to be successful. Pickering and Bunn (2007) see this as a negative to endurance hunting by erectus, and argue for passive 
scavenging in riparian forests where carnivore competition is low. However, if TWR began as early as I argue, larger-brained ${ }^{35}$ erectus could have had pretty good tracking skills.

The flushing and then tracking process is repeated until the animal is exhausted and cannot run any further or put up any defence. Only humans can physically handle running for hours through the hottest part of an African day, because of their morphology, breathe control, and capacity to dissipate heat by sweating copiously. After two or three hours the animal finally is at the end of its tether and one of the team of hunters simply walks up to it and clubs it on the head. Such hunting techniques would have been possible for larger-brained, robust erectus hominins, and would have dovetailed nicely with intentional pirating of wild dog kills, since they too are diurnal social carnivores that run large animals down in relay teams (usually around dawn or dusk).

\subsection{The Cognitive Significance of Carving Large Carcasses}

To reiterate: significant portions of Oldowan evidence indicate that large carcasses were being carved right from 2.6 onwards (Plummer 2004 for review). New evidence from the 2.3 mya site of Bouri reveals that size $2(25$ to $100 \mathrm{~kg}$ ) and $3(114-341 \mathrm{~kg})$ ungulates were the predominant mammal species taken, and cut-marks show that they were definitely captured and butchered by hominins before other predators/scavengers got to gnaw their discarded bones. They were also able to carve animals as massive as the extinct long horn buffalo, hippos, and giraffes. Task analysis shows that carving such large animals is in itself a major feat, even with modern steel knives: ask any experienced hunter.

Carving of such large animals requires co-operation even between modern hunters. Where I grew up no hunter went after the 400 to $500 \mathrm{~kg}$ moose alone unless they had a well-trained horse to help them manipulate the carcass, with a pack-saddle for the meat. My point here is if the earliest Oldowan hominins (habilenes) were smart enough to co-operatively carve such large beasts, carry that meat back to safe refuges, and defend it and themselves from other huge carnivores on the way, they must have been smart enough to read track-ways in order to track down and capture large animals by intentionally miring them in deep mud.

There is also a great deal of new evidence that proves the cores for stone tools were somewhat knapped at suitable sources, then carried to another site and used for the further knapping of sharp flakes and making cleavers. The distances we are talking about are

\footnotetext{
${ }^{35}$ Early erectus brains were $850-900 \mathrm{cc}$. Chimp's are around 450cc, as were those of the australopithecines.
} 
between 3 and $12 \mathrm{kms}$ (Toth and Schick 2010 for review ${ }^{36}$ ). Oldowans had a lot more anticipatory cognition than chimps.

To sum up my Oldowan big-game hunting scenario here: I think driving animals into swamps on a habitual basis would have been the earliest form of big-game hunting. Endurance hunting would demand greater tracking skills and a lot more energy expenditure, so it was probably a technique used by the more robust erectus, not habilis.

\subsection{TWR and the Hunting of Smaller Animals}

TWR is absolutely necessary when the species targeted is active only at night. A good example is the spring hare, a nocturnal animal that the !Kung San regularly track to their burrows during the day. They then hook them out with long barbed sticks: this smallish animal is easily obtained by such simple means and is therefore a mainstay of their meat intake. There are many examples of such effective hunting techniques for capturing smaller prey that could have been practiced by early hominins with such simple technology as bare hands, simple wooden spears, clubs, stones, and digging sticks.

Hill and Hawkes (1983) study of the simple but effective hunting techniques of the Ache is a very good expose of such methods. Forty percent of their meat intake is gained with no more than TWR, co-operative teamwork, and digging sticks doubling as clubs. Another ten percent of their fare is fish; these are all caught with their bare hands. Modern Africans still catch large catfish this way. I have caught eels and trout with my hands, using the 'tickling method'. It is quite easy to do once one develops a light touch, and is especially effective at night when they are resting under stream banks.

A method the Paiute (Nevada Indians) used for hunting rabbits was foolproof but extremely simple. Rabbits have to visit waterholes in the evening, so the whole tribe would encircle a water-hole at that time of day and slowly and silently close in on the drinking rabbits. Finally the gaps between band-members were so narrow that when the rabbits finally panicked and tried to escape they were easily knocked out with clubs and throwing sticks. Another simple method they used for capturing ducks was to wade very slowly through water at neck height with a mat of moss over the head, and simply pull the ducks underwater by their feet and crush their skulls with their thumbs (Gilmore 1953).

\footnotetext{
${ }^{36}$ These theorists posit Oldowans could have suddenly come up with such behaviours while still in a somewhat similar cognitive state to chimps. I do not agree with that view of course.
} 
The main point I want to make here is that knowledge of animal behaviour ${ }^{37}$ is far more important than technological complexity when it comes to many techniques of hunting. Therefore the means/ends cognition enabled by the beginnings of a narrative faculty could have made very simple and yet highly successful planned co-operative foraging methods possible even for very early hominins. With such methods they could have captured large quantities of small aquatic fauna and small terrestrial mammals.

I will now introduce one simple method of catching fish and other wetland fauna that can explain the origin of what [(Domínguez-Rodrigo and Martínez-Navarro) 2010, (Shipman, Bosler et al. 1981)] call "palimpsest sites". There are many Oldowan and early Acheulian sites containing bones and sometimes hundreds of stone tools, but there are no cut-marks on the bones. In Acheulian sites one can see that the hand-axes are still standing vertically in the sediment they were preserved in. Invariably these sites were in shallow water on the edges of lakes or in slow-running shallow feeder streams nearby.

When I was camped in the New Zealand wilderness and needed fresh protein, the easiest to procure was that from the native eel. The best way to catch them was to throw the bodies of a couple of skinned possums (a 1 to $3 \mathrm{~kg}$ introduced marsupial from Australia that became a terrible environmental pest) in a slow-running shallow pool in a nearby creek or river every day or so. All the eels from far downstream would come to feed on them. I could spear a fresh eel to eat whenever I liked. Catfish behave just like eels and sharks, they are scavengers as well as predators, strongly attracted to meat whether fresh or rotten. Fresh is best, because they can easily detect blood, and are as excited by it as sharks. Modern rural Africans still obtain a very large species of river catfish by this method.

I propose this is what hominins were doing at these sites: attracting catfish, snapping turtles, and small crocodiles up close to shores of lakes and shallow feeder streams where they could be easily speared or clubbed, by throwing the remains from other predators' kills in the water. Semi-aquatic cane rats would also have been attracted to such bait.

As I have said, hand-axes have been found still standing vertical where they somehow got stuck in the mud at some Acheulian sites. I posit they could be spun into water because of

\footnotetext{
37 "Hunters invest more in learning or reading the behaviour of the animal than in technology". William Laughlin, in "Man the Hunter" (ed. Lee and De Vore 1968, ch. 33., p 313)
} 
their shape ${ }^{38}$. Trying to stun or kill cane-rats, big catfish and small crocodiles with them this way would have been fun and good practice for juvenile Acheulians. Such baiting techniques are a forerunner to trapping techniques, and hence would constitute a sure sign of the anticipatory cognition already conferred by the narrative faculty on Oldowan hominin minds.

\subsection{The Cognitive Competences of Oldowan Hunters and Gatherers}

The simplest, most effective way to thwart scent-trailing predators is to wade into water and keep down-wind of them (there is always a breeze heading down streams at night). If the dangerous predator is a lion having a climbable tree nearby will provide safety; but if it is a leopard a tree will not. Refuges on alluvial islands or peninsulas dotted with climbable trees surrounded by water would have been a good option for early hominins (see illustration below: from flickR Photo Share website).

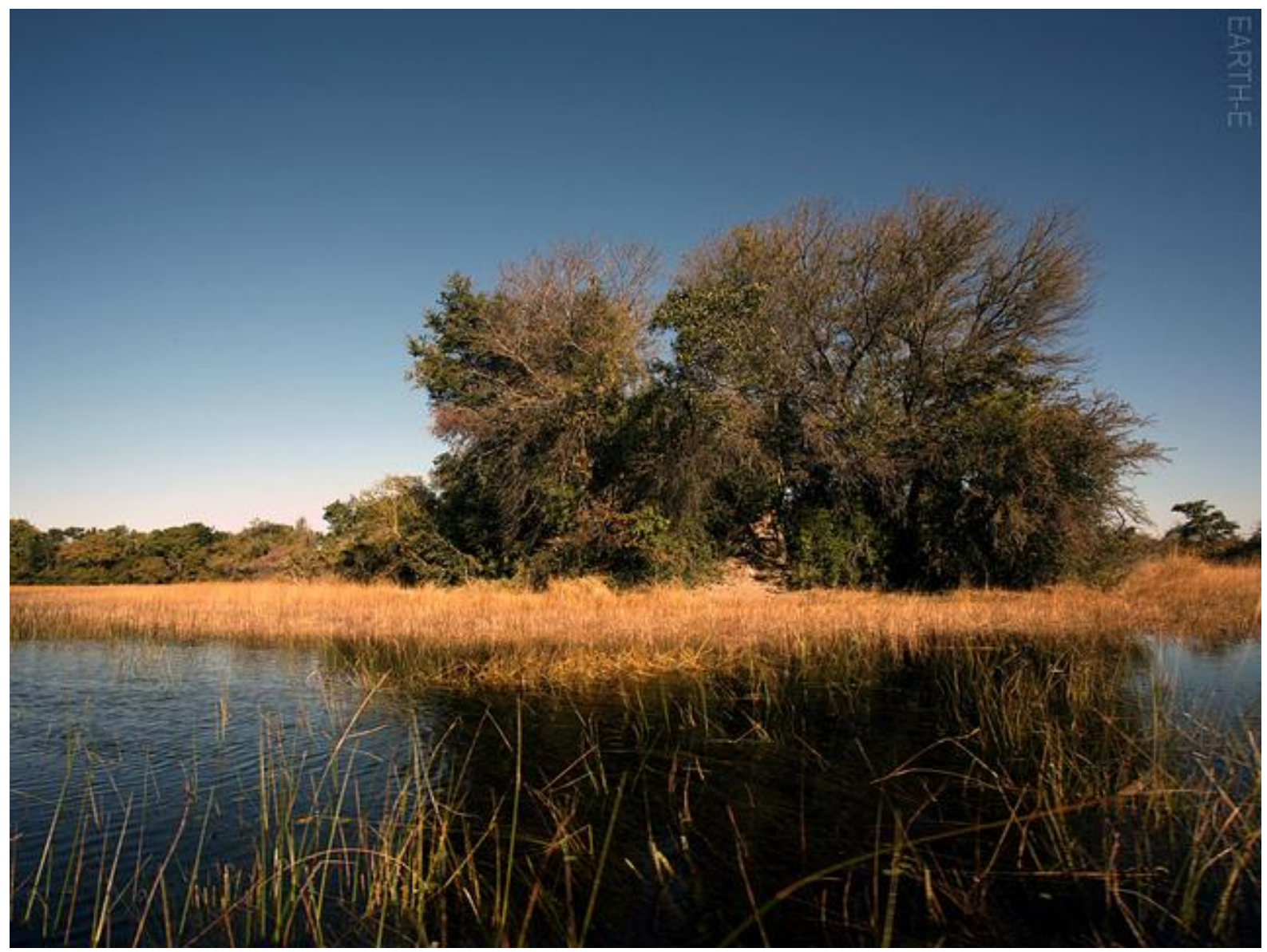

\footnotetext{
${ }^{38}$ Anyone who has ever tried to stun a fish in shallow water with an ordinary round stone (like myself) will be inclined to accept this speculative idea. The force of a thrown round stone is immediately nullified on impact with water. Hand-axes would cut through the water surface. Besides, eels and catfish will break the surface for food.
} 
Much paleo-archaeological evidence strongly indicates they were living in such environments. Plummer's excellent review of Oldowan cultural evidence (2004) provides a summary of the paleo-geography of all Oldowan sites between 2.6 and 1.8 Ma. They were all situated in lacustrine/riparian/alluvial shallow waters.

So TWR skills and ancillary narrative cognitive capacities would have conferred an over-all selective advantage when competing with other scent-tracking carnivores for prey, scavenging opportunities, or being able to dodge those creatures which predated on us. This might include other hominin bands, of course. Although Oldowan population densities might not have been very high in the beginning, because male chimps regularly patrol their territorial edges and go beyond them to actively hunt or confront other chimp strangers, I am assuming that early hominins probably exhibited this co-operative social trait as well. Meetings between bands of bonobos are usually friendly, but when whole bands of a hundred or more migrate to other parts of their 25 sq. mi. territories in cohesive units, the biggest males are at the lead in case they run into another troupe (McNeil 1996 citing SavageRumbaugh).

As track-reading hominins became more wide-ranging, such social interactions would have made TWR skills of paramount importance (not to mention the associated skills of hiding one's tracks from other hominins). Hence group selection for better narrative informationgathering from TWR and communication of that information within a troupe of hominins would have created an intra-specific cognitive arms race. Even if relations with outsiders were not hostile, you would still want to know if strangers were about, simply because they might be competing with you for certain resources.

My bet is that the first narrative communication of earliest hominins was mimed like the pretend-play of children, and it was often used to transmit TWR information to help others find or dodge other hominins and dangerous predators, and later to make hunting plans. By the advent of larger-brained true hominins (erectus) the greatest worry for a band with a good narrative faculty and TWR skills may have been other hominin strangers, not scent-trailing predators and scavengers. Knowing a stranger's footprints were not those of any member of one's band would have been highly significant.

All HGs without horticulture ( for example the Ache) were obligate nomads unless they lived in extremely resource-rich areas, simply because easily-caught game soon runs out in close proximity to a band of hungry hominins (Hill and Hawkes 1983, cited in 1985). The energy 
spent hunting and gathering rapidly becomes not worth the meagre returns. The best thing to do is move on to new territory for awhile and return when populations of game have recovered. Here again is another reason I think application of simple TWR skills in the social domain, re confrontations with strangers or other dangerous predators, may have been somewhat more relevant to our pre-Oldowan ancestral life-ways than TWR for hunting animals.

The best result of all to be gained from searching for new hunting grounds is to find territory where no other hominins have been, because the animals there will be much easier to capture. Hence there is a natural psychological 'gravity' of an impulse for exploration in all hunters and gatherers. Chimps do not exhibit this exploratory behaviour. Their territories are much more fixed, and they rely on resources lower in the trophic pyramid. Again, hominin exploratory behaviour would have instigated much more fission-fusion social complexity, with TWR being important for keeping track of band-members.

Of course areas seasonally rich in resources may have allowed temporarily larger social gatherings, but such dramatic fission-fusion social events in themselves would select for more narrative cognition in order to keep track of many reciprocal and confrontational relationships over time. This idea is highly orthodox, since it fits well with the classical social brain hypothesis.

Therefore the exploratory foraging, defensive and social advantages gained by extra TWR skills and their application should cause co-evolutionary selection for restructuring of archaic portions of the brain to entrench the HNF. This should naturally be followed by expansion of the plastically developmental neocortex for flexible storage and planned applications of the information obtained by the HNF from TWR (and all other natural signs).

The first habilene skull found, dated 2.3 mya, clearly shows expansion in the temporal and parietal areas (Tobias 1987),(Holloway, Broadfield et al. 2003). Its brain was twice as large as an ape brain relative to body size, as was the first erectus skull, which clearly shows further expansion in those areas, as well as the medial prefrontal cortex. These areas of the neocortex make up the core structure that supports default narrative mentalizing in the human brain (Schacter and Addis 2007; Addis, Pan et al. 2009).

This same core structure supports autobiographical memory, prospection, and theory of mind (Spreng and Grady 2009). The right parietal area is dedicated to reading the minds of others, 
and the left deals with past and future narrative scenarios concerning the self. All information entering the brain and during memory retrieval is ordered by the central ganglia or hippocampal/para-hippocampal zone. In all mammals this area deals with navigation in space. In hominins it also deals with navigation in time.

I posit social TWR navigation took the pre-hominin mind into the fourth dimension because TWR is all about when as well as who made the footprints making up a track-way. The timetravelling anticipatory cognition the HNF provided gave us a cognitive advantage over all other creatures. Humans narratively navigate in time on a default basis, by projecting the imagined self into past and future scenarios (Buckner 2007). People with Alzheimer's Disease and dementia, who have lost their self narrative and therefore cannot function in society, cannot find their way about in novel topographical environments either.

Human navigation through novel territory is not possible without physical, ego-centric selfreferentiality. Most other animals do not possess this objective physical self-referentiality to any degree ${ }^{39}$. This is why they hate leaving their home territory (for example, pig-dogs very often get lost if they get too far away from their master). The same principle applies for mental-time-travel. Think of time as an ocean: the mental self-referentiality of the autonoetic self-narrative is our anchor in the past, our keel in the present, and our steering device towards the future. Without it we are soon lost or 'all at sea'.

All of the above supports the strong claim that if early hominins had not entered the TWR cognitive/behavioural niche humans would not have evolved. Contemplating TWR's possibly colossal relevance to why we are the way we are, an obvious question arises given (a) orthodox niche-construction theory, and (b) that ours is the only lineage on this planet that has ever exploited track-ways as natural signs. Why have so few cognitive evolutionary theorists ever seen the possible evolutionary import of the unique cognition demanded by TWR till now?

\subsection{Reasons for Previous Theoretical Disregard for TWR}

No other theorist besides the naturalist/Jungian philosopher Paul Shepard (1996) and myself appears to have explored to any depth the possibly very early cognitive evolutionary relations between TWR, culture and communication, even though there is fairly wide consensus in

\footnotetext{
${ }^{39}$ However, mature great apes and dolphins do pass the mirror-recognition test. I posit this occurs because both needed 3-D physical self-representation in space, the apes because they are arboreal, the dolphins because they are fully aquatic. This may have been another necessary but not sufficient pre-adaptive cognitive trait of our Ardipithecan ancestors.
} 
some theoretical camps that all true hominin species have been hunter-gatherers (HGs) for at least 2 million years.

The most obvious reason relates to the fact that both Shepard and I grew up tracking and trapping: it is due to a very understandable lack of experience because of modern life-ways. Paul Shepard (deceased 1998) grew up in the swamplands and waterways of Missouri (U.S.A) helping his family trap muskrat for a living. Most theorists who have discussed modern HG ethnography re hunting skills and the evolution of human cognition have never had any practical experience of using TWR.

Another reason may be that quite a few HG tribes have been using dogs for hunting, some for several thousand years. Perhaps this is why Hewes (1994) suggested that we did not learn to use TWR until behaviourally modern sapiens began hunting with dogs. However, domesticated dogs do not appear in the archaeological record in the Old World until between 26 and $15 \mathrm{ky} \mathrm{ago}^{40}$ (Leonard, Wayne et al. 2002). Usually they have been intentionally bred and trained to hunt only certain species of animals. And make no mistake: the hunter that uses dogs will only take them where he has seen track-ways and other signs of the animals in question, or someone else has seen such signs and given the hunter the information needed to make the effort worthwhile.

The crucial point here is that hunting dogs have to be used efficiently, for they require a lot of intentional breeding and training, as well as feeding when not hunting. They cannot be allowed to hunt for themselves, for they soon return to a feral state of uncontrollable behaviour and end up killing domesticated animals (and sometimes even attacking humans). The use of dogs for hunting is a very modern cultural phenomenon.

In fact most dogs in historical HG tribes were largely kept around camp to warn of approaching danger since their hearing and sense of smell is better than ours by far. They were also useful for cleaning up old bones, rotting meat-scraps and human toddlers' faeces. And they made a good fall-back source of protein when times were tough, animals were scarce, and starvation threatened (dog meat is still regularly eaten in some cultures).

Dogs cannot practice self-inhibition unless exhaustively trained and under the here-and-now command of their owner. During the tracking and stalking by humans of many types of

\footnotetext{
${ }^{40}$ The data is very controversial, some kind of symbiotic 'tolerated theft' arrangement could have been in place significantly earlier, but dogs certainly weren’t domesticated millions of years ago.
} 
animals dogs are worse than useless for they tend to get too excited and noisy, especially when used in packs. This is why they are only used to hunt certain species that will quickly bail up and confront them, or immediately climb a tree. The dogs then surround the animal and make feinting attacks or bark at the base of the tree to keep it in situ until their human master can get to the scene and dispatch the target animal.

We also need to remember here that human foraging has had such huge ecological impacts that the targets of ethnographically known foraging are almost certainly very different from the targets of early to mid-Pleistocene foragers. The African animals that lend themselves to the use of dogs to capture them are large and/or fierce and dangerous because they bail up and face their attackers (kudu, buffalo, and warthogs for example). As far as I know these species do not regularly appear in the archaeological record until 400 to $500 \mathrm{kya}$. This is a similar date to when the javelin appears in the archaeological record so perhaps there is a connection here.

Now back to theoretical disregard for TWR. Another reason may be that many HG tribes live in jungles where track-ways are not nearly as obvious to ethnographers. However, all HGs are track-ways readers by 'second nature', including those living in closed forests ${ }^{41}$, for just a single foot-print in a damp bit of ground on a forest trail can convey a great deal of highly salient information. Terrestial animals in thick forest or scrub largely keep to well-travelled routes or 'game trails'. Therefore one only needs to see a few prints now and again where the trail crosses damp spots to discover whether a targetted animal has heard you and is bolting or is still 'cruising', how far ahead of you it is and so on.

Another more generalized reason TWR's possible relevance to hominin evolution has not been fully explored may be due to the 'no thought before language' paradigm that has been so prevalent until just recently in certain theoretical disciplines ${ }^{42}$, which believed that language could not have emerged till $45 \mathrm{ky}$ (when symbolic behaviours first appeared, according to a very Eurocentric point of view, now discredited). The few ethnographers and evolutionary theorists who have extensively studied modern HG tracking skills, and have

\footnotetext{
${ }^{41}$ They augment TWR by proceeding in utter silence and using directional listening for audial clues; mimicking other animals (mating calls for example) is also a good method for getting them to approach within killing distance (see Botha and Knight 2009). The Ache are excellent jungle trackers: Hill and Hawkes (1983) however said they could not see the tracks and other traces they were following.

${ }^{42}$ Personally I have always found this idea extremely bizarre. So have the builders, artists (especially filmmakers), hunters, sportsmen etc. that I have happened to mention it to. How could we forget all the daydreaming we did when young, and still do as adults? Why do all the visual media have such a powerful effect on our minds and emotions? Our mentally simulated narratives often are and always were very visually mimetic.
} 
thus realized how cognitively demanding full-blown TWR can be, may have been influenced by this 'given wisdom'. They therefore posited that such cognitive skills could only have been a recent acquisition: that is, around $45 \mathrm{ky}$ (for example Hewes 1994, Liebenberg 1990 ${ }^{43}$, Noble and Davidson 1996).

Yet another reason may be that many cognitive evolutionary theorists believe, because of 'simple' Oldowan and Acheulian technology, that we must have been restricted to scavenging large animals before around $500 \mathrm{ka}$, and hunted only small animals habitually. Whether our ancestors did or did not actively run down large game before $500 \mathrm{ka}$ is somewhat irrelevant to my thesis anyway: many smaller animals, especially secretive nocturnal species, can only be found by TWR techniques by a carnivorous primate with no scent-trailing ability. So by task analysis alone the very weak hominin olfactory sense makes habitual TWR and application of its information by habilis/erectus hominins a very safe bet.

Many theorists however, especially evolutionary psychologists, have never doubted that hominins were fully fledged HGs from at least 2 mya onwards. The recent evidence of 3.4 mya cut-marked bones (McPherron et al 2010) from an animal as large as a cow indicates we should probably assume they were on their way to being cognitively capable of a very simple HG life-way well before $2 \mathrm{mya}^{44}$. I think the reasons these theorists have been disregarding TWR are the most understandable (as compared to those already given) and there are three. None of them had ever realized that TWR was (a) so informationally rich; (b) cognitively demanding; and (c) ecologically and socially so salient (Sterelny pers.comm).

In my opinion the most telling reason is (c) for it is also true of Paul Shepard (1996): they had not seen TWR's potential socio-ecological orienteering role. Otherwise the penny would have probably dropped due to its obvious relevance to the highly orthodox social brain hypothesis. Here we have arrived at the crucial addition to Shepard's powerful insight into the hominin triggering track-ways story. It is crucial because it provides the final missing piece of our cognitive evolution puzzle: our ancestors initially entered the TWR niche in the social domain.

I argue this triggered the incremental evolution of the HNF and associated elsewhere-andwhen social competences. A million or more years later these competences made it possible

\footnotetext{
${ }^{43}$ I repeat: Liebenberg (2008) now thinks erectus was a persistence hunter, and therefore must have had reasonably good TWR skills.

${ }^{44}$ This is one of the reasons I posit habilene/erectus only started regularly obtaining large mammals because they had to due to environmental contingencies.
} 
to habitually and intentionally apply TWR in the extractive foraging domain by cooperatively hunting large animals in a very goal-directed, narrative/predictive manner. What my readers need to remember here is that there is no lower or upper limit to the cognitive acuity demanded by exploitation of track-ways, from very basic to extremely fine-grained. Fully developed TWR and its informational use in hunting is both a social 'character-reading' endeavour and an intellectual, scientific pursuit of natural history knowledge.

Scientists are always talking about 'tracking' various phenomenological events. In the terminology of cognitive linguistics, 'tracking' is a dead metaphor: it is such a universal human way of finding out what is happening in the real world, it has become an unconscious 'way of speaking' in every language you may care to mention. One might even say TWR potentially demands the intellectual capacity for inductively understanding everything that has happened unobserved in the real world based on empirical evidence, including what the mouse droppings lying next to the nibbled cheese in the your cupboard might mean. TWR is not an all or nothing capacity.

At various points I have reiterated that we must have entered the TWR niche in the social domain, mainly because the HNF is above all a faculty for social cognitive competences. Now that we have explored the cognitive demands and possible applications of TWR when hunting other species, we can begin to understand why social TWR markedly increased prehominin social complexity, thus triggering the evolution of the hominin narrative faculty.

What we will discuss in the next chapter is (1) where, when and why only our ancestors entered this unique cognitive-behavioural niche; and (2) how it affected the pre-hominin social domain. Then we will identify (1) the great ape cognitive/behavioural traits that provided a window of opportunity for entering the social TWR niche; (2) how simple social TWR triggered the evolution of the hominin narrative faculty.

$* * * * * * * * * * * * * * * * * * * * * * * * * * * * *$ 


\section{Chapter 4. Social TWR and the Hominin Narrative Faculty}

\subsection{The Laetoli Fossilized Footprints}

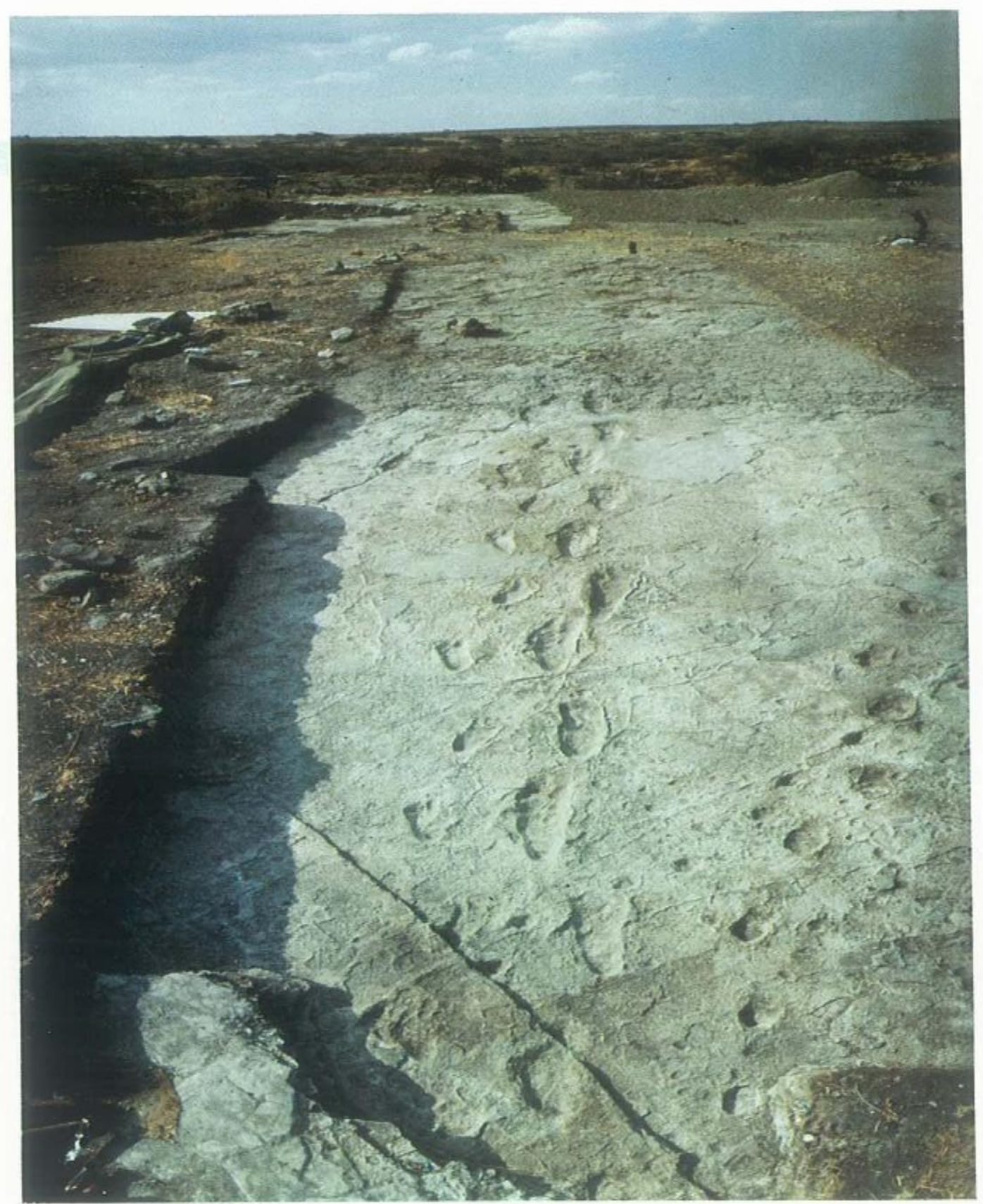

View of the excavation at Laetoli Footprint Site $G$ at the point where threc hominid trails are intersected by two Hipparion trails. (Photo by John Reader.) 
About 3.6 million years ago three of our pre-habilene ancestors walked across a flat area bordering an upland lake in part of the Rift Valley of Eastern Africa now called Laetoli. Their footprints (along with those of many other animals ${ }^{45}$ ) were perfectly recorded in $10 \mathrm{~cm}$ of volcanic ash, deposited in several layers by a nearby volcano that had been erupting every few hours for the last fortnight. Very soon afterwards a thin layer of limestone bearing sediment was laid over the ash which indelibly 'cemented' all extant footprints into fossilized track-ways.

These hominin track-ways were rather fortuitously found in 1976, on the way back to camp from an adjacent dig, by an archaeologist working with Mary Leakey. He was bending over to pick up a piece of dried elephant dung to throw back at one of his companions. He thus saw the indentations of one or two of the recently exposed fossilized footprints due to the angle of the fading evening light.

The Laetoli Track-ways are undoubtedly one of the greatest of all paleo-archaeological finds, for they proved incontrovertibly that hominins were fully bipedal by such an early date, well before the earliest (until very recently) evidence of stone tools at $2.6 \mathrm{my}$. But they also tell a story (like all track-ways) that provides us with some understanding of their perpetrators' cognitive capacities.

As we can see, although two adults and a juvenile made these prints (69 mostly perfectly preserved prints over 27 meters), at a cursory glance there appear to be only two sets of prints, those of one adult and one juvenile, walking side by side and hand in hand (because the gap between them is so consistently the same). This is because, as a close inspection later revealed (by Alan Root, a wildlife photographer and an expert tracker), the slightly smaller adult was stepping quite exactly in the prints of the larger (Harris 1987).

Like all track-ways they are extremely informative, and especially so in this case because of their age. We are going to tease out that information in the next two sections, and this analysis can be taken as another example of full-blown TWR cognition. First we will deal with what they tell us about the physical and ecological attributes of their authors, and then discuss what they reveal about these hominins' cognitive capacities.

\footnotetext{
${ }^{45}$ All these animals were going to the lake nearby for water. Rabbits were the most plentiful species, followed by ostriches (Leakey 1979).
} 
I then explain why only our great-ape lineage entered the TWR niche. This is followed by a description of the increase in social complexity that was the inevitable result, and caused selection for better social TWR skills. The nature of track-ways demanded the co-evolution of a unique cognitive faculty for social elsewhere-and-when narrative imagination, which later made more intentional systematic tracking of other hominins possible.

I next turn to the evolution of the HNF, beginning with delineating the great-ape social competence that made an initial entry into the social TWR niche possible: the capacity for here-and-now true imitation. I then argue that it was this capacity plus great-ape here-and now intention reading skills or incipient TOM (theory of mind), combined with the continual exploitation by hominins of their own personal track-ways that made the evolution of autonoetic (self-knowing) autobiographical narrative memory possible.

I defined the concept of autonoesis (Tulving 1985) in my introduction (section 1.0). For example, autonoesis is the sense of self that enables children over the age of three to know that they are pretending to be someone else. I repeat: without this autonoetic sense of an intentional personal past there can be no sense of an intentional personal future, and therefore none of the mental-time travel required for anticipatory cognition for instance (Suddendorf 1997; Corballis and Lea 1999; Suddendorf and Corballis 2007).

Without the autonoetic sense of an intentional self travelling from the past into the future there can be no awareness that others have autonoetic intentional selves, and therefore no narrative faculty or any of the elsewhere-and-when uniquely hominin social competences. I present my model of the hominin narrative faculty and the four cornerstone elsewhere-andwhen social competences in chapter five, and then present developmental evidence that the narrative faculty emerges between the ages of three and four in all normal humans. I also present comparative evidence that it is this faculty that turns the here-and-now social competences of chimps and children younger than the age of three into elsewhere-and-when competences of post four-year old humans.

Most cognitive theorists and neuroscientists would agree that "self knowingness" or autonoesis (Tulving 1985), which equates with episodic (also coined by Tulving) or autobiographical memory is a cognitive trait peculiar to the hominin lineage (Suddendorf, in Corballis and Lea 1999, Suddendorf and Corballis 2007 for review). To reiterate: in his review of developments in the human evolution debate since 1985, Tulving (2001) states that the evolution of human autonoetic episodic/autobiographical (=what I call self-narrative) 
memory was obviously the cognitive key to the evolution of all hominin culture and communication. The next two chapters in particular fully support that view, and explain why social TWR was the trigger for the evolution of autonoesis.

My impression after much research is that no cognitive evolutionary theorist has directly addressed the central problem of when and how this autonoetic or episodic/autobiographical cognitive capacity for an intentional self-narrative began to evolve, and why only in our great-ape lineage. I believe this is because it has been widely assumed that it could only have emerged much later in our evolution. Most theorists, Dennett ${ }^{46}$ included, think it could not have been in place before linguistic language. I argue it is the key to our unique social competences, including the language capacity; that it has been evolving incrementally ever since we entered the social TWR niche; and that it was already somewhat entrenched in the mind/brain of the hominins who made the Laetoli track-ways 3.6 million years ago.

\subsection{Physical and Ecological Attributes of the Laetoli Hominins}

The Laetoli hominins' track-ways show they were obligately bipedal, since their feet were almost completely 'humanoid', with a non-opposable big toe. They made prints nearly identical to those made by the habitually unshod feet of teen-aged modern HGs. Although many theorists have claimed that australopithecines could have made these prints, fossil evidence of their foot structure indicates a still somewhat opposable big toe, useful for grasping branches when climbing trees, and quite a bit shorter than the other toes (Tuttle, Webb et al. 1990; Tuttle, Webb et al. 1991) (see illustrations next page).

I tend to agree with Tuttle, but on the other hand, I think the adults' prints look more flatfooted than the Machiguenga teenager's foot (see page 74). The prints do not look like they were made by the feet of endurance runners, because they do not indicate a high arch or instep. We discussed endurance hunting by erectus hominins in my third chapter: it requires systematic TWR skills and therefore a fairly well established narrative faculty. Therefore I conclude these Laetoli hominins were probably still simple/opportunistic social trackers in the main, not yet systematic trackers of other animals.

When I first saw Laetoli Track-ways illustrations several years ago it immediately occurred to me that since (1) the juvenile was undoubtedly hand-in-hand with one of the adults; and (2) a juvenile primate would be far more likely to be travelling hand in hand with its mother, it is

\footnotetext{
46 In his book 'Consciousness Explained' Dennett describes autonoesis, or 'consciousness', as "the center of narrative gravity".
} 


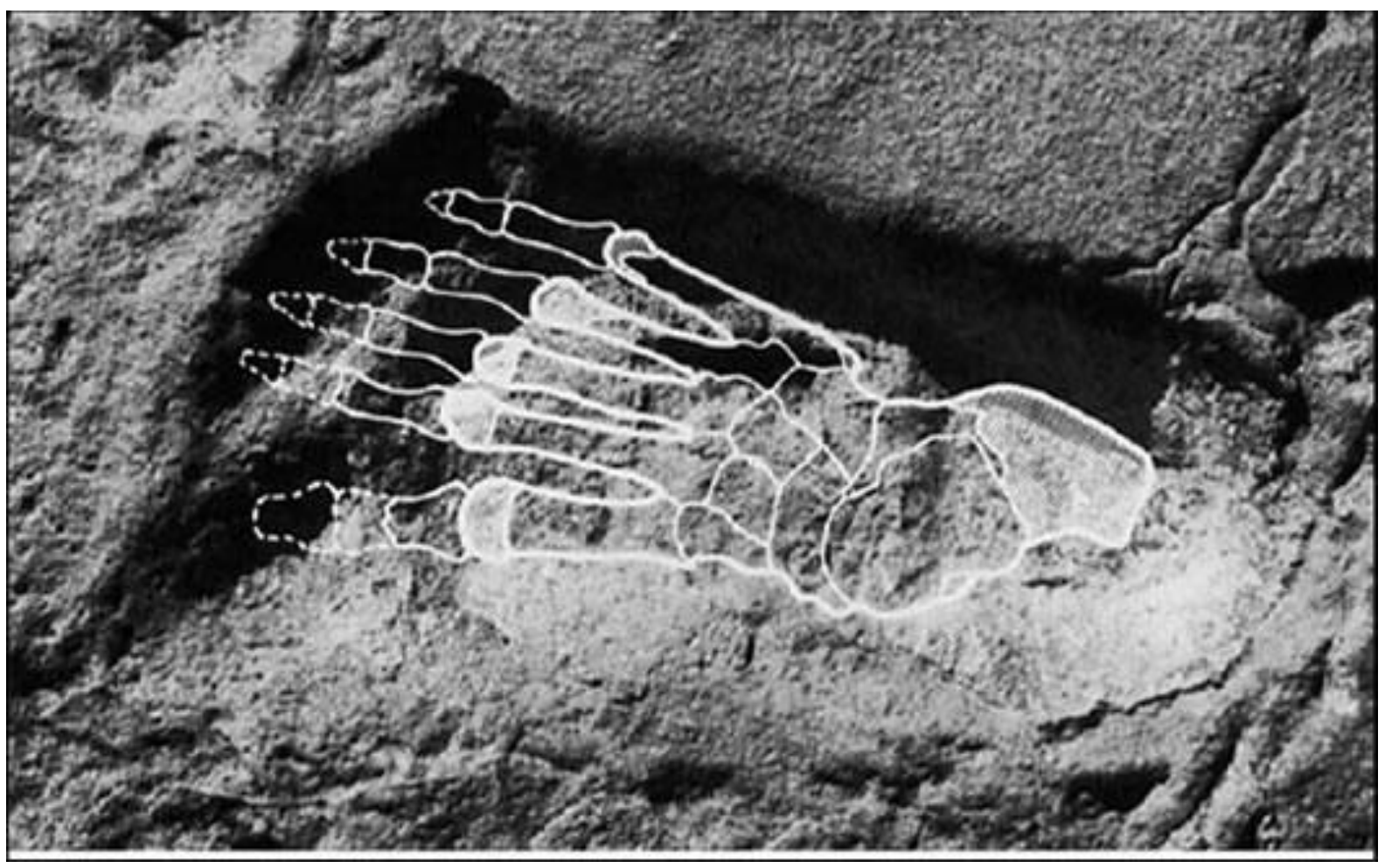

How could an australopithecine hind-foot with this skeletal structure make the above foot-print?

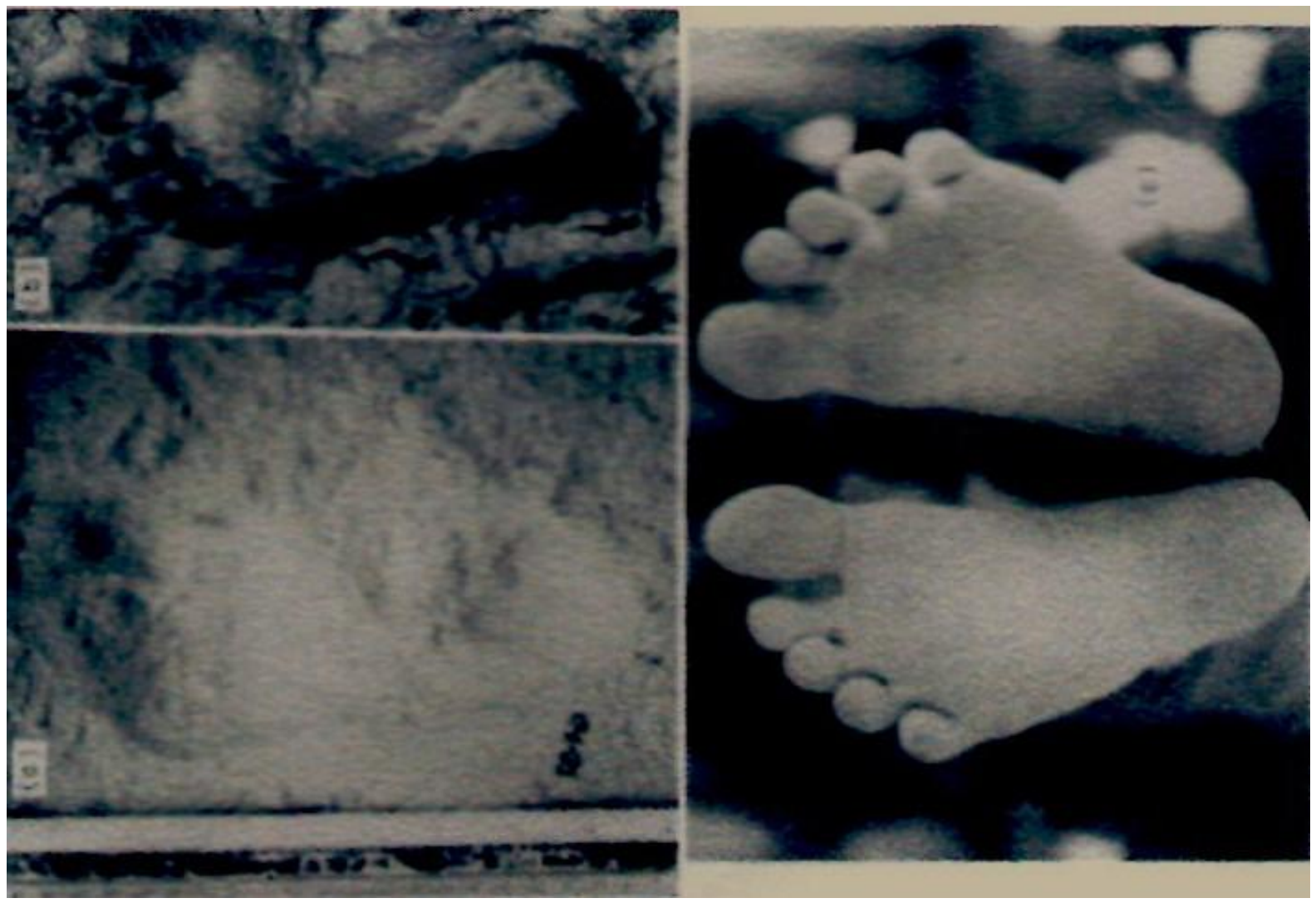

From Tuttle, Web et al (1990): In illustration immediately above: (a) is Laetoli print, (b) is Machiquenga teenager's print, and (c) is a photo of his habitually unshod feet. 


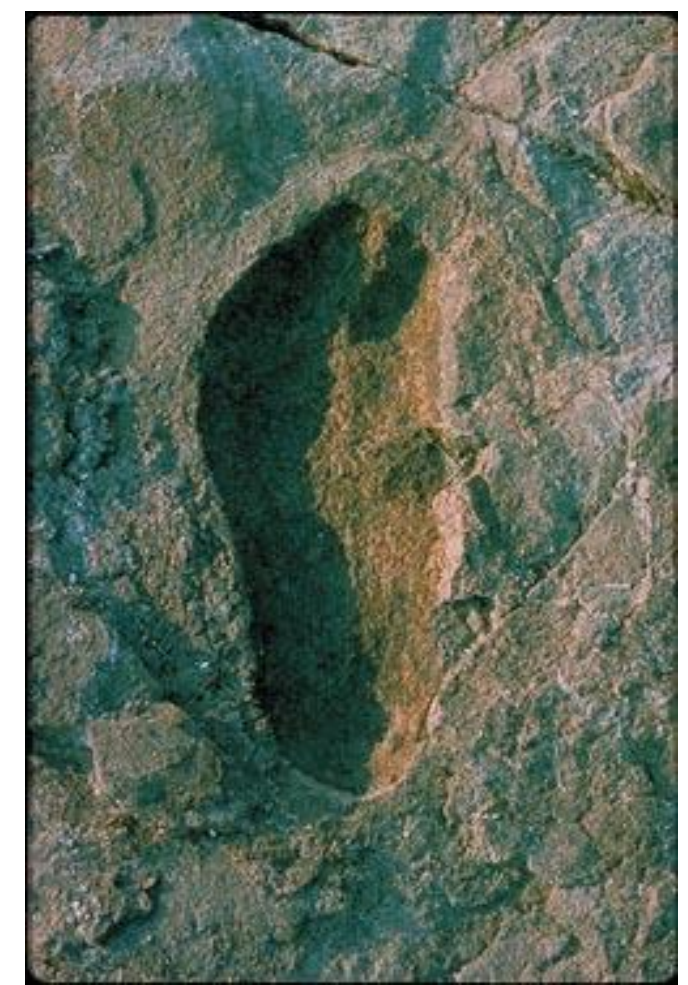

Juvenile's left foot clearly shows non-opposable big toe and flat-footedness. However, young children do tend to be more flat-footed than older humans.(From Modern Human Origins website, photo by John Reader).

extremely likely that the adults were male and female, with the male travelling first. As we can see, difference in adult print-size was minimal.

Since the size of one's feet usually corresponds to the size of one's body, this indicated to me that sexual dimorphism must have already been much reduced. Australopithecines are known to have been much more sexually dimorphic than habilene/erectus hominins. I decided they were not made by australopithecines for that reason alone. Ardipithecus (4.4 mya) is now known to have already been minimally sexually dimorphic, with very reduced male canines when compared to both chimps and australopithecines (White et al 2009, Suwa et al 2009), so my earlier track-ways interpretation has been thoroughly vindicated.

In sum, the above TWR physical information indicated to me that some very habilene-like hominin species was extant at 3.6 mya, a million years before Oldowan Culture emerged. Therefore I began to explore the idea that our ancestors were on the way to some sort of cognitive and neurological re-arrangement well before 2.6 mya. Four years later evidence of cut-marked bones dated 3.4 mya has just been found (McPherron et al 2010). I repeat: the 
cut-marked bones were from a cow-sized animal and the nearest source of suitable stone was $6 \mathrm{~km}$ away. This certainly indicates cognitive advancement beyond the ape mind-set.

I also noted that these track-ways were found on what had been an open highland watershed on the shore-line of a lake (=lacustrine environment) at the time they were made. It therefore occurred to me that the Laetoli hominins might have been habitual foragers of wetland flora and fauna, given that much of that fauna does not require sharp stone tools to procure or be made edible, especially thin-skinned catfish and easily opened shellfish.

As it turns out, we now know the back-molars of 4.5 mya Ardipithecus were already quite small, indicating a far more omnivorous diet than the more herbivorous australopithecines, which had larger molars than habilus/erectus. This strongly indicates the smaller molars of the latter true hominins are a primitive feature: they did not evolve because our ancestors suddenly starting eating large quantities of meat after 2.6 mya.

What all this new evidence adds up to is that some habilene-like hominin, already much smarter than any other ape, must have been around long before 2.3 mya, and probably had a staple diet of wetland flora and fauna, just as I had conjectured. My theory has also been further confirmed by incontrovertible evidence that hominins were eating large amounts of catfish, turtles, and crocodiles at 1.95 mya (Braun et al 2010) and at other earlier sites (Verhaegen and Puech 2010, Stewart 1994 for full reviews).

My speculative theorizing about the 3.6 mya hominin life-ways has always been based on the Laetoli Prints, in addition to: (a) all early hominin fossils were preserved in wetlands or floodplains, and stone and/or bone caches were deposited in water or very near to it; (b) my own first-hand knowledge of how much easier it is to live by hunting and gathering near wetlands or estuaries, due to their resource richness, in the form of fish, shellfish, edible succulent plants, bird's eggs and so on.

I also knew the nutrients from a fish and shell-fish diet are far more high-quality than those gained from meat (in fact their quality as 'brain food' is only equalled by those obtained from brains and marrow-Broadhurst et al 1998). This wetlands diet idea was then quite unorthodox, but quite a few theorists have since realized such a staple diet would have more than adequate to fuel an expanding brain, in combination with bird's eggs, fledglings, and small aquatic mammals such as cane-rats. 
Shell-fish are easy to open, catfish are so easy to catch with the bare hands even bonobos can capture them. Modern Africans still procure them this way. They have thin skin with no scales and therefore would have been easy to eat for a fangless hominin without sharp tools. In short, the idea of a stable diet of wetlands fauna and flora for early hominins is rapidly becoming quite orthodox (Cunnane and Stewart 2010 for review of the literature). ${ }^{47}$

I have described this speculative journey because it is a very good example of the information to be gained from reading the track-ways and other signs left behind by a species, without ever seeing it in the flesh, combined with practical knowledge (folk biology) of the typical ecologies of differing environments. I posit erectus was able to migrate throughout the warmer parts of the Old World by 1.6mya (at the latest) because their mind/brain was already capable of this kind of folk-biology plus a degree of systematic TWR cognition. In short, I think they were a lot smarter than envisaged by many other evolutionary theorists. As we shall now discover, the 3.6 mya Laetoli Prints also provide crucial information about their authors' cognitive attributes.

\subsection{Cognitive Attributes of the Laetoli Hominins}

It is obvious to any tracker that one of the adults and the juvenile were walking in parallel very close together, but not close enough to be arm-in-arm: they were holding hands. The adult in question was most likely the second adult who had slightly smaller feet, because as I pointed out in section 3.1, a juvenile is more likely to be travelling close to a female, probably its mother. Female hominins usually have smaller feet than males. It is highly significant therefore that Mary Leakey (Harris 1987) stated that it looked as if the juvenile's prints were made later than the other dual track-way ${ }^{48}$.

If the juvenile's footprint was made later then the second adult holding the hand of the juvenile must have been following the first adult's track-way sometime later than it was made. Here we have arrived at the most crucial consideration regarding the cognitive attributes of our 3.6 mya Laetoli ancestors. It is the lynch-pin of the chronology of my triggering track-ways theory.

Anyone who has ever tried to do what the female (let's take the leap) was doing, continuously stepping exactly into someone else's footprints, will know how difficult a

\footnotetext{
${ }^{47}$ These theorists, however, think it was a fish and shellfish diet that caused the brain expansion of hominins. I do not agree with such a simplistic scenario, of course.

${ }^{48}$ There would undoubtedly have been a great deal of input to her reading of the ancient story told by these track-ways from the expert tracker Alan Root.
} 
behaviour it is to maintain, especially over such a long distance. The difficulty arises because it requires prolonged concentration or mental detachment from other here-and-now motivations, and a fine-grained bodily self-awareness, especially if the leader is somewhat larger and therefore has a naturally longer gait. Mary Leakey and her fellow paleoarchaeologists tried to imitate the second adult hominin back at camp and came to the same realization.

Alan Root reported (Harris 1987) that he had seen very young chimps doing the same thing when playing 'follow the leader games'. This is the only incidence of this behaviour I have found mentioned in the primatology/comparative psychology literature. So either chimp observers have taken no notice of it (which I doubt very much because it is a very human-like behaviour) or it is a fairly rare phenomenon, in which case it is evolutionarily inconsequential (in the case of chimp evolution, not ours).

Very young chimps do spend a lot more time in the bipedal mode than adults, so I suspect there is something important about the combination of being able to see one's own feet at all times, plus the inherently greater complexity of four-footed track-ways, that makes it easier to cognitively exploit bipedal prints. It is certainly obvious that one must be able to see one's own feet to do what these young chimps and the smaller Laetoli adult hominin were doing.

On my first reading, because it was reported that the juvenile's prints looked as if they were made later, I decided that the adult female (?) was literally following in the adult male's (?) footsteps some time later both for safety and in order to find him. Then my supervisor (Sterelny pers.comm.) commented that one does not need to step so exactly into footprints for safety, so safety is unlikely to be the only motivation.

I therefore began to think, and still do, that this emulative/imitative behaviour would constitute the simplest, cognitively least demanding form of opportunistic social TWR, and could have evolved out of the here-and-now imitative cognition young chimps must possess in order to step in each other's prints in the here-and-now. I am suggesting here that adult Ardipithecans had commandeered this young ape behaviour a million years before the Laetoli Prints were made, for the sake of safe passage through featureless boggy wetlands. We will discuss this idea further in the next section.

On the other hand she might have only been stepping in the first adult's prints so exactly because of the utter strangeness of the volcanic ash she was walking in, and normally would 
not have bothered to do so when searching for someone by following their track-way. It could not have been because the ash was still hot, for the following reasons. Leakey (1987) describes one point where the juvenile's prints appear to lag slightly and turn to the left. She described her vision of the juvenile pausing slightly to look at something interesting in the distance to the left quite poetically.

I think what this means is they were in no hurry, so the as was not hot, and the female was slowly and very cautiously stepping exactly into the footprints in front of her. When pairs of modern !Kung San hunters head off in the morning at a fast trot, whoever is behind places his feet fairly exactly in the prints of the 'trail-breaking' front man (Silberbauer 1981). They say they do this for two good reasons: only the front hunter has to concentrate on not snapping twigs (which would scare prey) or stepping on thorns with his feet (which would make any further jogging very painful) and the man behind does not.

Why one slightly smaller adult hominin at Laeotili was stepping so exactly into the tracks of the larger hominin 3.6 mys ago is most parsimoniously explained in this way, for they were walking in $10 \mathrm{cms}$ of very recently deposited volcanic ash at the time. However, the discoverers of these track-ways have stated the juvenile's prints were made later, and they had an expert tracker with them, Alan Root.

Since he was a professional wildlife photographer, I for one do not doubt his tracking skills. The juvenile could not have been travelling on his own, because in that case his track-way would not be so consistently the same distance from the dual track-way. If he had been, I bet his prints would have been in the dual prints as well. I also think if the female had been at the leader's heels, her prints would not have been so exactly in his (?), simply because she would have felt safer in his (?) company.

The problem I have, being a seasoned tracker myself, is this: the juvenile's prints might just look fresher than the dual prints. A sure sign of freshness is sharply defined outlines, since the edges of prints tend to crumble as they dry and become more blurred after an hour or so. The dual prints might look older because they were more blurred at the edges due to the impossibility of achieving a perfect match when stepping in another person's prints.

What is needed is a modern forensic examination of to see if there is any material between the first and second adult prints, such as ash (it had been falling intermittently for two weeks) or pollen. It would only require damaging one dual print, after all. If there is any material 
then (1) the material will be identical to what is under the juvenile prints; and (2) she will definitely have been following the leading adult sometime later in order to find him.

Now that the new evidence of cut-marked bones at 3.4 mya has turned up (McPherron et al 2010), my TWR interpretation of these famous footprints has become more positive ${ }^{49}$. As I stated in section 3.1, the Laetoli hominins must have already possessed some semblance of the narrative faculty in order to be so much more cognitively anticipatory than chimps. This indicates to me that goal-directed searching for another band-member by stepping in their prints was already passé for our Laetoli 3.6 mya hominins. Therefore normally she would not have bothered to step exactly in someone's prints when trying to follow their track-way in order to find them. She was doing it purely for safety. This last version of our Laetoli trackways saga is now the one I think most likely, given that the juvenile's track-way was made later.

It is extremely likely that the Laetoli hominins had never experienced such a strange phenomenon as volcanic ash before. The female would have been unable to see what was underneath, so walked slowly and as exactly as possible in the footprints of the absentee adult. For our purposes however, no matter how one interprets the evidence, the Laeotili fossil track-ways indicate very robustly indeed some ancestral adult pre-hominins were cognitively exploiting conspecific track-ways to some degree before 3.6 mya. I think they were probably doing so a million years before this date, for reasons presented in the next section. The question that arises now of course, is why only our great-ape lineage entered the TWR cognitive-behavioural niche

\subsection{Why Only the Hominin Lineage Entered the Social TWR Niche.}

Chimpanzees in the wild exhibit incipiently many aspects of human behaviour and cognition, including making tools like simple spears for impaling bush-babies in hollow trees (Pruetz and Bertolani 2007), assembling nut-cracking stones, and fishing-rods for termites (but they do not use tools to make other tools). They also regularly kill and eat smaller creatures such as colobus monkeys. They exhibit flexible group hunting behaviours analogous to that any pack of wolves, wild dogs, or lionesses engage in, learnt by trial and error. However, the only

\footnotetext{
${ }^{49}$ Others might think one cannot assume the Laetoli hominins were the same species that cut the bones, because they think there were probably quite a few hominin species around (Sterelny pers. comm.). I will make two points in reply: (1) whichever species cut those bones, it was probably smart enough to follow conspecifics' track-ways in order to find them; (2) if there were so many other species of hominins around, why hasn't fossil evidence of their very humanoid, fully bipedal feet turned up? Many theorists class co-existing australopithecines as hominins. I do not, partly because of the very humanoid nature of these footprints.
} 
instances in which they appear to exhibit what appear to be truly goal-directed hunting behaviours occur when they are hunting conspecifics.

When a band of males patrols the edge of their territory looking for chimp strangers (which they will sometimes attack and kill; they also eat infants, but not adults) they exhibit true group cohesion, maintain a communal silence (they will glare at a companion who makes a noise) in order to be able to hear unsuspecting strangers foraging. They also take note of signs such as old nests and faeces of other chimps, which they sniff, just any other mammal would. The one thing they never do, however, is what any human would automatically do: look for footprints of strangers. This is a highly significant difference, for like all primates they are very good at visually reading patterns, due to their exploitation of very patchy resources such as fruiting trees. Here is where the question 'why us' naturally arises.

I think the answer has three major components. The first and most obvious is perceptual: besides the same excellent pattern-reading visual primate sensibilities as other apes, our earliest ancestors must have had the same impoverished olfactory sense compared to most other mammals, which use scent and/or sound as social and territorial markers in the main. Even chimps have a better olfactory sense than ours, and obligate bipedalism would have physically removed ground-level scent trails from hominins' olfactory capability. Olfactory perception would have been lost, on the 'use it or lose it' principle.

The second is morphological and behavioural: they were fully bipedal with a very humanoid foot structure, more suited to walking and running (plus wading and swimming) than climbing, by at least 3.6 my ago (as the Laeotili Footprints prove). This means they must have spent much more time than any other ape on terra firma, for australopethicine fossil foot bones indicate a more opposable, shorter 'big toe' still somewhat adapted to an arboreal lifeway.

A recently discovered almost complete fossil skeleton of a 3yr old female A. afarensis (dated 3.3 mya) has a gorilla-like shoulder structure, its hyoid bone (part of the throat structure) shows it had laryngeal (throat-area) air-sacs like all other great apes except for hominins, and its hand and foot bones indicate a still very arboreal life-way (Alemseged, Spoor et al. 2006). 
There is said to be "some evidence" of bipedality ${ }^{50}$. The skeleton is embedded in sandstone, so delineating it for full study will take a few more years of laborious work.

In the case of the complete hind-limb of a 4.4 mya Ardipithecus ramidus fossil, there can be no doubt left that this likely ancestor to both hominins and australopithecines was fully bipedal but still had a foot useful for arboreal activities (Suwa, Asfaw et al. 2009; White, Asfaw et al. 2009). Its big toe was far more opposable than those of the later australopithecines. One would assume then that the Ardipithecans still nested at night in trees in their socio-ecological niche in upland closed forests, but foraged in open lacustrine/riparian/alluvial floodplains and swamps during the day within those forests.

The bipedal stance would also have made tracks more salient, for the head is above the feet, not in front of them as with four-legged creatures. One can see one's feet making prints if one is bipedal. It is being bipedal that makes exact matching of footprints at all possible. Only young chimps in the bipedal mode have ever been seen to match foot-prints. An increase in field of view could make patterns of prints more noticeable. Another factor would be that if dependent for mobility on only two legs, where one places each foot becomes highly salient (especially when carrying heavy loads). Tripping and falling on river stones and/or spraining an ankle is threatening to survival for an obligate bipedal species. When one is travelling at some speed (jogging) 'watching your step' becomes even more vital.

The third reason early hominins entered the social TWR niche is possibly the most important: all the archaeological evidence prior to the mid-Pleistocene (stone tool and bone 'caches') indicates that our ancestral preferred environment was lacustrine (lakes, ponds, swamps) since the split from the last common ancestor. Recently discovered fossil sites of the earliest facultatively bipedal Ardi hominins, from 5.8 Ma onwards, have all been found in what were open lacustrine swamps and flood-plains within highland closed forests at the time of deposition (White, Asfaw et al. 2009). Unlike in the deep forests chimps tend to live in, trackways would be continually present in the mud and sand of swamps and lacustrine/alluvial beaches.

Tracks do not show up nearly as often in closed forests, and most animals, including chimps travelling on the ground, use the same well-defined game trails for navigation. The firmness of these well-travelled trails means they do not record foot-prints, especially of creatures with

\footnotetext{
${ }^{50}$ This recent evidence (especially the lack of laryngeal air sacs) also leads me to conclude that they should perhaps not be called hominins any longer, as they are in much of the literature.
} 
foot-pads (instead of hooves), unless they happen to cross damp places. However damp places in deep forest are very often dodged when animals are on the move to new foraging spots because the thickness of the understory makes them difficult to traverse. Therefore most game trails are on the drier ground of ridges, where travelling is much easier.

The question naturally arises here as to whether the lacustrine scenario emerges simply because fossils are more likely to form in soft muddy substrates. I think this is unlikely for four reasons:

1) All evidence of hominin stone technology and butchering activity is found in such sites up to the mid-Pleistocene (including the recent 3.4 mya evidence)

2) Most modern and historical HGs have preferred to live in or near, and to forage in, resource-rich lacustrine, riparian, or estuarine environments

3) Stone artefacts would not have needed to be in such substrates to be preserved, yet all early finds are situated in such substrates

4) Many of the features of hominin morphology are exhibited only by aquatic or semi-aquatic mammals: lack of olfactory glands, sweating, tears, hairlessness, fatty enlarged mammary glands, and no laryngeal air-sacs (unlike all other great ape species, which I think has to be highly significant, as an indication of underwater activities). ${ }^{51}$

Now our excellent primate pattern reading abilities need to be mentioned, but chimps have this capacity as well of course. I think the above reasons constitute why they never entered the TWR niche: they have always lived in a different socio-ecological niche.

\subsection{The Social Significance of Track-ways.}

All hominids' footprints and track-ways are individually recognizable, especially in small bands. Age classes (toddlers and juveniles) walk differently from each other on smaller feet than adults; adult females have smaller and more slender feet than males; more slender lighter people have correspondingly different foot-shapes from larger bulkier people. Within these gender, age, and morphology categories, everyone has slightly differently shaped feet from

\footnotetext{
${ }^{51}$ I think a balanced version of the semi-aquatic ape theory of how we became fully bipedal through wading and foraging in watery environments should be taken more seriously by theorists of human evolution. It already is by several : e.g. Stringer (1997), Verhaegen and Puech (2000),Tobias (2010). Tobias once championed the 'out on to the savannah' hypothesis. However he has now accepted the semi-aquatic/lacustrine view, after reviewing all the evidence collected over the years of a life-time of work in paleo-archaeology.
} 
everyone else, with telling asymmetries between feet, idiosyncratic variations in gait, angles of heel strike, and so on (see a sample of footprints below. From flickR Photo Share Website)

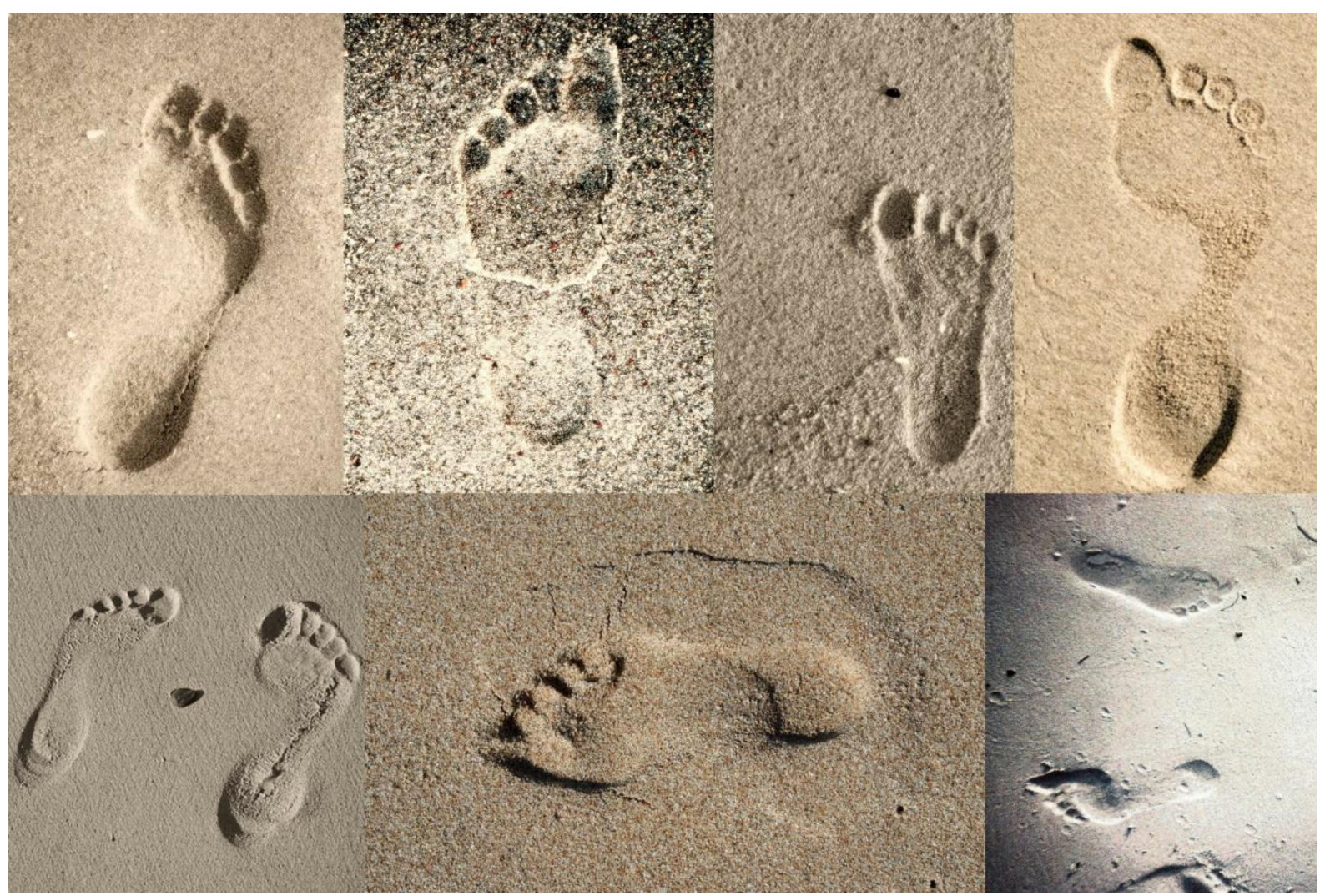

I repeat: (1) San children know their mother's footprints from all others by age four without being taught how to recognize them; (2) Silberbauer (1981) recounted how Gxwi San hunters laughed out loud when asked if it was possible to recognize human footprints because they thought the question was absurd; (3) track-ways of all larger animals are just as recognizable to a skilled tracker (Liebenberg 1990, Silberbauer 1981).

Being a tracker myself I can state that in the case of humans one can almost always discern their age and sex (if they are travelling bare-foot). Even if they are shod one can easily tell whether they were just wandering and enjoying the view or headed in a definite direction, actively hunting or fishing by a river, or laden with a pack- even whether they were drunk or not. I have once or twice amused myself by reading the stories of what went on, under the cover of darkness, told by human track-ways on the beach by a motor-camp the next morning. 
When one is going river-fishing or hunting, the very first signs one looks for are fresh tracks of other humans; quite simply because 'the early bird gets the worm' and tracks made by others on the same day means it is probably not worth foraging in that area. If there is no 'free territory' left in the vicinity, then one has no choice but to go 'further in' or beyond where any other human has been if one wants to find whatever one is foraging for.

Right from the beginning of our ancestral entry into the social TWR niche the most important track-ways for hominins exploring new terrain (besides their own personal trackways and those of allies, alloparents and offspring) would have been track-ways of hominin strangers whose prints they did not know (as well as those of dangerous predators). In short, conspecific track-ways would have been a very important social marker (as canine scentposts and scent-trails are social markers). It is crucial here to remember that one's own recognizable footprints are very important indeed, especially when foraging in boggy areas where one cannot see any defining landmarks on the horizon.

The best way to find one's way back to firm ground is to back-track, stepping on your old prints. One does this quite naturally with other people's prints as well: I did this as a child without being told to, when my father began to take me duck-hunting around swamps and ponds in marshes. When foraging alone it is taking notice of one's own track-ways that makes safe and efficient navigation in open featureless wetlands at all possible. This idea of continually taking note of one's own prints is very important to my thesis, as we shall discover in section 4.6.

\subsection{How Social TWR Increased Hominin Social Complexity}

Since all human footprints and track-ways are so individualistic as to be instantly recognizable by all members of the same band, and some times last for several days or weeks (unlike scents), the possible social complexity ramifications of using footprints as a social marker (as cats and dogs use scent) within the daily argy bargy of the earliest hominin social domain are enormous. I believe no other version of social complexity theory has ever come up with a good reason why the social domain of the earliest hominins should ever have become more complex than that of our fission-fusion Machiavellian chimp cousins.

Any hominin with the ability to recognize and read conspecific track-ways would have increased fitness with regard to Machiavellian motives; consider, for instance, dodging energy-consuming confrontations with stronger enemies by vacating areas where their fresh 
track-ways can be seen. And vice versa for setting up optimal co-operative social encounters. A sub-dominant hominin male with incipient TWR skill would be able to choose to follow the recognizable track-way of an attractive female only when the alpha male's track-way is nowhere to be seen near hers.

Being able to follow and find one's band when lost or left behind due to environmental catastrophe (such as a flash flood or a shower of volcanic ash), or find a strayed ally, sexual partner or child in silence when a dangerous predator or other hominin band was nearby, even if it was raining 'cats and dogs', would also be very useful. Tracks will last for quite some time in the rain; almost all scents are instantly wiped out by water. This is why land-based carnivores are loathe to hunt in the rain or in very wet swamps, and are so obsessive about remarking scent posts in their home territories. Being able to dodge dangerous local predators on the basis of recognizing their track-ways would also increase fitness (remember my cougar story: I never went near that copse of trees again).

All the above is plausible, and would also apply to foraging and co-operative interactions. The evolutionary upshot is this: even the simplest cognitive level of TWR, having to literally step in the old tracks of another individual hominin in order to maintain mental concentration, or a working memory of them when trying to find them, would be selected for through both sexual and group selection in the social domain alone. However I think initially they began stepping in each other's prints and their own old prints purely for safety and orienteering.

The semi-aquatic wetlands socio-ecological niche of the earliest hominins plays a central role in my hypothesis here. I argue that stepping in one's own and others' old prints is what made living and foraging in such environments at all possible. Therefore entering the social TWR niche at such a simple level immediately increased fitness. Even simple TWR of social partners both allows for a more complex form of fission-fusion organization (because you can assemble/separate/reassemble in more complex ways) and because you can know something of what others have done out of eyeshot. In effect, this elsewhere-and-when knowledge constitutes something like gossip.

Imagine coming upon the month-old tracks of someone who had since died, for instance, one of your alloparents say. Or imagine a young male finding a track-way of the female he has been currently cohabiting with heading off into the reeds beside the track-way of his closest male ally or a more dominant male. I think it is extremely obvious that social TWR would 
have amped up the social complexity of early hominin societies to an exponentially greater degree than the fission/fusion society of chimps.

The narrative cognitive capacity to figure out what others had been up to from their old trackways, during the periods when you hadn't been able to see them, would have been heavily selected for. However, I think the narrative faculty for intentional tracking of other hominins took quite some time to evolve after the initial entry into the social TWR niche.

\subsection{The Initial Entry into the Social TWR Cognitive Niche}

I think somewhere between the 4.4 mya facultatively bipedal Ardipithecans and the 3.6 mya obligately bipedal Laetoli hominins our ancestors began habitually stepping into conspecific prints for safety and orienteering because they also had become obligate foragers in open featureless wetlands. Exploiting conspecific footprints for safety and orienteering when foraging is ecological of course, but it would also be social when foraging with others, or refinding one's band when foraging alone. There is a seamless gradient between the two domains. I accent the social aspect in my thesis because I think the HNF and the hominin elsewhere-and-when social competences had to have evolved in the psychological 'crucible' of intimate relationships between the self and important others.

The most intimate relationship of all is one's relationship with one's self, of course, and one's own old footprints are like one's shadow, impossible to ignore or get away from. They would have had a continual mirroring effect, bringing the personal past to mind for any track-ways reading hominin. This is a crucial consideration I return to in depth in the next section, for I think it can explain how autonoetic autobiographical memory evolved. Right now I want to limit our discussion to the initial hominin entry into conspecific track-ways exploitation: stepping into someone else's prints when walking at their heels.

Such a here-and-now imitative cognitive effort in physical self-awareness and muscle control could have been possible given a still chimp-sized brain, since (1) we have one anecdotal report of juvenile chimps stepping in each other's prints when playing bipedal 'follow the leader' games; and (2) adult chimps are capable of true associative here-and-now imitation. They also have some here-and-now theory of mind or intentionality-reading capability. This makes sense, because the two competences are intimately related throughout all social interactions between self and others [(Whiten 2008), (Tomasello 2008) for reviews]. 
I believe these two capacities, when combined with here-and-now stepping in recognizable absentee conspecific footprints on a habitual basis, triggered the evolution of the elsewhereand-when hominin narrative faculty (HNF). To follow right behind someone and step fairly exactly in their footprints is 'here and now' or associative true imitation. There is no impetus towards elsewhere-and-when cognition in such behaviour. As time went on stepping in absentee conspecifics' footprints for safety and orienteering would have developed quite naturally. This would have opened the door to elsewhere-and-when cognition.

I argue stepping in prints when the author of those prints is nowhere to be seen or heard would constitute the beginning of elsewhere-and-when or decoupled true imitation, because everyone walks differently, with different gaits, angles of heel-strike, and so on. Therefore one has to be imitating the physical mannerisms of an absentee author of a track-way to some extent when matching one's prints to theirs, not just copying the product of their actions, which is emulation.

Some theorists may disagree, but given the idiosyncratic nature of track-ways, I am still inclined to insist matching prints with another absentee hominin is not just elsewhere-andwhen emulation of the product of an action. Although that would be cognitively sophisticated in its own right, as Whiten makes clear, the emulation/imitation dichotomy is probably best viewed as a continuity anyway (Whiten 2000).

I therefore hold that true elsewhere-and-when imitation of the past actions of an absentee conspecific is what is occurring. That is precisely why matching prints exactly is difficult. Prolonged true imitation demands mental concentration and a fine-grained physical awareness of one's own body. So far we are talking about physical as-if role playing, requiring an autonoetic physical sense of self. But mental concentration means detachment from other here-and-now motivations, and here is where our window to the sense of an intentional self and others as intentional selves opens.

It opens because of the nature of track-ways. Since track-ways are immediately and inexorably directional they appear to be made by an intentional being carrying out various activities in a goal-directed manner. They tell stories about what their authors have been up to, or aiming for, in the past. Hominin prints and track-ways are also easily recognizable.

Since male chimps sometimes spend a lot of time alone, and a female and a male sometimes disappear to be on their own together for a few days, we can assume that early hominins did 
the same. For here-and-now minded chimps, they would be 'out of mind' for the rest of the troupe over the time period in which they were absent. This would not remain true for very long for early track-ways-exploiting hominins.

Even if in the beginning absentee band members' footprints were only opportunistically stepped into for safety or orienteering, hominin track-ways are recognizable, and therefore can tell stories about what specific, well-known individuals had been up to. Now for two crucial points:

(1) Stepping in an absentee conspecific's track-way of footprints would constitute acting out the story of what they had been up to in an as-if role playing manner, using the physically self-aware cognition needed for exacting imitation.

(2) This scenario would often be repeated with one's own old recognizable trackways, precisely because track-ways are so durable.

Since stepping in old track-ways for safety and orienteering would have definitely increased fitness, the above cognitive scenario would have been happening on a daily basis. In fact, I think stepping in one's own old track-ways would have happened every day in every way when foraging. Some of my readers might be saying here, but this is foraging or ecological TWR, not social TWR.

I have already said the gradient between social and ecological navigation is seamless. The main motivation in extractive foraging is to obtain whatever resource one is foraging for and then get it back to the safety of home base as quickly and efficiently as possible. If hominins were already co-operative breeders to some extent, as Ardipethcans' lack of male canines would indicate, home base included other band members that were depending on that resource for their well-being. This kind of extractive foraging is all about social motivation.

\subsection{The Self-Mirroring Effects of One's Own Track-ways.}

To reiterate: one's own foot-prints are like one's shadow, impossible to get away from, have a 'mirroring' effect on what one has been up to in the past, and yet unlike any scent-trail, can be extremely decoupled in time from your present self as their referent, simply because of their much greater durability.

Most modern humans have never spent any time alone in the wilderness hunting and trapping. Therefore they cannot know just how important one's own old track-ways are when it comes to efficient daily navigation. This is especially true of open, featureless 
environments such as swampy wetlands covered in tall grasses and reeds, river-flats covered in shrubbery above head height, or dry-land flat areas covered in bush or scrub where one cannot see defining landmarks on any near horizon.

As the angle of the sun changes during the day, or cloud cover ensues and then departs, even the very same scene you saw an hour ago can look completely different due to the effects of light on foliage. A wise hunter pauses often to look at where they have just been in order to more easily recognize it on their way back to camp or home-base. However there is not a hunter born who has never been temporarily lost, or what all hunters call euphemistically, "turned-around", especially in the excitement of the hunting and stalking itself. For the animal you are tracking leads you on its own possibly winding journey to wherever you finally track it down, stalk it, and kill it.

Hunters are also continually looking for new territory where there is possibly more game, so they are often in somewhat unknown surrounds before they find an animal track-way worth following. The most changeable environments of all are wetland and riparian/lacustrine environments, due to continually changing water-levels, which rise with every rain-fall, and lower during every dry period. We are talking of changes that can occur over just a few hours here.

Another factor that anyone who has not hunted large game will not be aware of is this: when you are loaded down with a heavy bundle of meat or the gutted carcass of a largish animal, finding the quickest and easiest pathway home is vital. To get lost or 'turned around' then is exhausting, and extremely risky in environments where there are dangerous predators and scavengers which are very strongly attracted to the smell of blood. I believe this is why menstruating females were kept isolated from everyone else in many HG societies (Woodburn 1964, 1982 for example). ${ }^{52}$

The upshot is that back-tracking home using one's own past track-way becomes the bottomline modus operandi for returning hunters. One also steps in or very near one's old prints for the sake of safety when carrying heavy loads. This would also be true for early hominin gatherers in featureless wetland environments, of course. One's old track-ways also tell where one has already been, and this too is very important information. When foraging

\footnotetext{
52 This is probably why that famous eccentric in Alaska who tried to live with grizzlies finally got eaten: for the first time ever he took a girl-friend in with him for his usual three month stay. About a month later they both got eaten. Prey animals are also easily alarmed by the smell of blood: another good reason for the social isolation of menstruating females.
} 
iteratively in the same territory, I cannot count the times I have suddenly come upon my own week-old track-way and realized I had already foraged in that particular area, and therefore it was a waste of time to carry on in the direction I had been heading.

When trapping or snaring one is continually setting traps or snares in new territory (since there will obviously be more game in non-trapped areas). Therefore one is continually using the track-ways one made yesterday in order to re-find those trap-sites when checking them the next day. When gathering one is always using one's old tracks to re-find a particularly good berry-patch, or bed of water-cress in a swamp, and so on. One is also continually exploring new territory when gathering, not just when hunting.

Scenting animals are very restricted when exploring because their own scent is so ephemeral; so they loathe leaving their home-territories when foraging. Ask any pig-hunters: they will tell you their dog-packs are forever getting lost when they take them to new territory. ${ }^{53}$ The upshot is that foraging early hominins exploiting their own track-ways had a huge navigational advantage over all other scenting foragers, especially in changeable wetland environments.

But the main point I want to get to here is this: exploiting one's own track-ways on such a continual basis means that the stories they tell are continually mirroring what one has been up to in the past. The self-tracking hominin discovers that they had stopped to pick a handful of berries just there, later stopped to check an old track-way of a deer over there, later still stopped to climb a small tree to get their bearings there, and so on. They would also be reminded of what their mental states were at the time, especially when combinations of their old track-ways and those of other band members (or predators: remember my cougar story) told stories about agonistic or positive interactions with other band-members. Similar stories would be told by combinations of one's most intimate band-members' track-ways, as well.

Therefore I posit that entering the social (socio-ecological if you prefer) TWR cognitive niche triggered the evolution of the autonoetic awareness of an intentional self travelling through time, and therefore an awareness of other hominins as separate-but-similar timetravelling intentional selves. This awareness of one's own (and therefore others') timetravelling intentional mental states is the cognitive key to the autonoetic self-narrative at the core of the hominin narrative faculty, which created the 'spin-off' hominin cornerstone social

\footnotetext{
${ }^{53}$ Some pig-hunters put radio collars on their dogs in order to be able to find them again more quickly.
} 
cognitive competences. This autobiographical self-awareness also makes second-order intentionality possible.

\subsection{The Evolution of Dennett's Second-Order Intentionality}

Second-order intentionality is the cognitive capacity to know that you know what someone else doesn't know, because they weren't present when you had your personal experience of a certain event. It requires autobiographical memory of your own experience of the "who was where and when' of that event, which dovetails with an elsewhere-and-when theory of mind. In other words, one must be able to imagine being 'in the mind-and-body' of your own past ${ }^{54}$ selves in order to have autobiographical memory. Only then can one imagine being 'in the mind-and-body' of the past and present selves of someone else ${ }^{55}$.

Dennett (Dennett 1987) stated that how second-order intentionality evolved in the hominin mind was the hardest problem in human cognitive evolution to solve, because all primates spend most of their time in the company of most members of the troupe. One individual's experience is nearly always simultaneously everyone's experience, or very soon is (consider vervet monkeys' specific alarm calls for eagles, snakes and, leopards). Anyone who is physically absent to a here-and-now mind is very soon mentally absent to that mind. Chimps do have some here-and-now theory of mind (TOM), and human toddlers younger than three have much more. They are good readers of the intentions of others in the here-and-now. This is first-order intentionality, which all higher mammals possess (we will revisit this territory in the next chapter).

I argue here-and-now TOM evolved into the elsewhere-and-when TOM required to imagine what someone's mental states of desire had been when reading their old track-ways. This elsewhere-and-when TOM became second-order intentionality when following one's own old track-ways, because this is how the autonoetic sense of an autobiographical self-narrative evolved. Without the faculty for remembering one's past personal experiences, which includes what one's desires were at the time, one cannot know that one knows something and how or why one knows something. If one does have this autonoetic/autobiographical faculty, one can know that someone else does not know something one's self knows, and why:

\footnotetext{
${ }^{54}$ If you can have autonoetic autobiographical (=narrative) memory then you can imagine yourself having experiences in the future. This enables mental-time-travel (Suddendorf and Corballis 2007).

55 As I have said before in an earlier footnote, I am a simulation theorist when it comes to theory of mind capability, not a 'theory-theorist'. There is not the space here to discuss this material.
} 
because one can remember they weren't there when one had the relevant personal experience. Only an autonoetic mind can possess second-order intentionality.

When autobiographical memory or autonoesis evolved it allowed intentional trial-and-error auto-rehearsal through comparison of past personal experiences of success or failure. It also allowed intentional, autonoetic emulation/imitation of absentee conspecifics, or true pretendplay. I argue pretend-play is the developmental signature of possession of the narrative faculty, and is based on this cognitive competence. Even highly enculturated apes have only once or twice exhibited behaviour that could be interpreted as pretend-play.

Pretend play is based on acting out simple narratives, and as we know, all track-ways tell stories. We also know that the way a tracker reads these stories is by imagining being 'in the minds-and-bodies' of the creatures making them. We identify with the characters in a novel or a film in the same manner. I suggest the earliest hominins started developing this remarkable hominin faculty through imitatively stepping in each other's track-ways and their own recognizable track-ways as above. As the narrative faculty evolved, imitatively stepping bodily in another's tracks in order to keep them in mind when trying to find them became unnecessary. Tracking hominins became able to narratively imagine being in-the mind-andbody of the individual that made the recognizable track-way being followed.

The foot-prints of one's child or one's mother (including alloparents) are the most important track-ways of other band members during the developmental years. Here is our psychological motivation for intentional rather than opportunistic social TWR to evolve. I am also still digging here for the evolution of elsewhere-and-when mind-reading skills out of the incipient here-and-now mind-reading skills of all great-apes. Reading the mental states of one's most intimate absentee others would be continually triggered by the else-where-and-when mental representations demanded by track-ways analysis, in a co-evolutionary manner. Hrdy has pointed out the important fact that with many primates, young juveniles are never out of the sight of their mother; and the very young are never out of physical contact (Hrdy 2009).

If reproductive co-operation is ancient in the hominin lineage, these primate characteristics were not true of our ancestors ${ }^{56}$. Therefore finding and re-finding one's caregiver was important in such a fission/fusion socio-ecological niche. The psychological need to know where one's child or parent/alloparents were at all times would have driven social TWR skills

\footnotetext{
${ }^{56}$ We probably had no fur for the young to cling to at a very early stage in our evolution, if we were the semiaquatic ape.
} 
from opportunistically simple towards intentionally systematic and narratively speculative. As I made clear in chapter two the higher cognitive levels of TWR are not possible without the narrative faculty.

The above scenario would dove-tail with developing an autobiographical awareness of one's own intentionality through reading one's own past track-ways. Neural entrenchment of the autobiographical narrative memory needed for mental-time travel and the other social competences, triggered by reading the stories told by combinations of track-ways that included your own past interactions with others, was a definite possibility given the durability of track-ways plus social footprint recognition.

In sum, the every day in every way use of conspecific track-ways in the social domain would have led to the evolution of the narrative faculty for imagining agents intentionally acting towards goals in the elsewhere-and-when. There are many ways to capture small animals, simply but very successfully, without exploiting their track-ways. This is certainly true of aquatic fauna, and they of course do not make track-ways.

But I am including using old-track-ways to re-find good foraging spots in open, featureless edaphic flood-plains and swamps, or back-tracking one's way to home bases using one's own prints, in my picture of early, very simple TWR. The selective advantage of these skills is obvious; it was probably this ability to navigate using conspecific foot-prints that made foraging in such environments possible for a non-olfactory ape.

\subsection{Summary}

Now we can understand: (1) why entering the social TWR niche caused so much more social complexity, creating a cognitive arms-race for better TWR skills, thus triggering the evolution of the hominin narrative faculty; and (2) why I put so much weight on the idea that TWR must have started in the social domain: the HNF is above all a uniquely hominin social faculty. I think hominins were tracking each other long before they had enough of the narrative faculty to begin goal-directed tracking of other animals in order to hunt them down.

The Laetoli Fossilized Footprints indicate that by 3.5 mya some hominins were capable of intentionally tracking each other. The 3.4mya cut-marked bones (McPherron et al 2010) also point clearly to an anticipatory, narrative cultural and communicative capacity. Hominins were already a lot smarter than chimps. Their smartness depended on their capacity for intentional planned co-operative behaviours, and as I have laid out in this chapter, this 
capacity must have been conferred on them by the HNF and associated elsewhere-and-when social competences.

In the next chapter I present my models of the HNF and the four cornerstone cognitive competences, and present developmental and comparative evidence that shows: (1) these capacities of the hominin mind/brain constitute a qualitative difference from the ape mind; (2) they begin to emerge in all normal human children between the age of 3 and 4.

$* * * * * * * * * * * * * * * * * * * * * * * * * * *$ 


\section{Chapter Five: The Hominin Narrative Faculty and the Cornerstone Social Competences.}

\subsection{Introduction to the Models}

This section presents and briefly explains my models of the hominin narrative faculty and the four cornerstone social cognitive competences (illustrations next page and the following). Section two concentrates on the social competence for narrative communication, and hence discusses the relevance of the HNF to language invention. The following sections present developmental and comparative evidence of (1) the qualitative uniqueness of the HNF and the cornerstone social competences, (2) their developmental emergence in all normal humans, and (3) their phylogenetic and ontogenetic origins.

Take the cougar-tracking story presented in my preface for an example: elsewhere-and-when cognition was necessary to read the combinatory narrative of my past-self's track-way and that of the unseen cougar. My twelve-year-old self was momentarily pretending to be 'in the mind-and-body' of an earlier self and the cougar's past-self. I could not do this without an autonoetic sense of an intentional self that could know it was projecting that same sense of self into the cougar's mind, as if the cougar were an intentional being like me. I also could not be writing these words in the here-and-now without being able to remember that autobiographical experience. It is part of my self-narrative.

As I write I can see the pristine clarity of sunlight on the sparkling snow, the gloom under the spruce trees, the steep bare hill towering behind them. At the centre of this envisioning process the 'motif' image of one of the cougar's 'huge' footprints keeps re-occurring. But in my 'mind's eye' I see a substitute cougar for the one I never saw at all. That image is of a rather tatty, disgruntled looking cougar I saw two years later in a zoo on the other side of the planet. Behind that image looms a sort of 'ghostly' image of some huge fierce cross between a cougar and a sabre-tooth tiger, probably because my mind has recently spent so much time imagining the world of the Oldowan hominin.

In this manner our autobiographical memories are always being partially re-constructed, and new narratives are continually being embedded in the larger on-going self-narrative. Readjustments continue as we mature, because new experiences add nuances to old stories, and newly-gained semantic knowledge is added to the creative amalgams of images as well. A lot of narrative mentalizing is about pretending, or 'as-if' substitution of characters (for example my substitute zoo cougar) and events, in order to make sense or provide narrative continuity. 
My point here is our autobiographical memories are not episodic like photograph albums, with gaps between the photos.

We have incorrigibly narrative minds. The brain automatically fills the gaps between 'snapshot' episodes with images from other places and times to create a 'flowing' narrative. Baddley calls this functional part of 'episodic'/autobiographical memory the "episodic buffer" (Baddeley 2000). This 'buffer' is also used for 'episodic' constructions/simulations of the future. I think we should call the whole 'episodic' faculty the narrative faculty (see below).

\section{The Hominin Narrative Faculty}

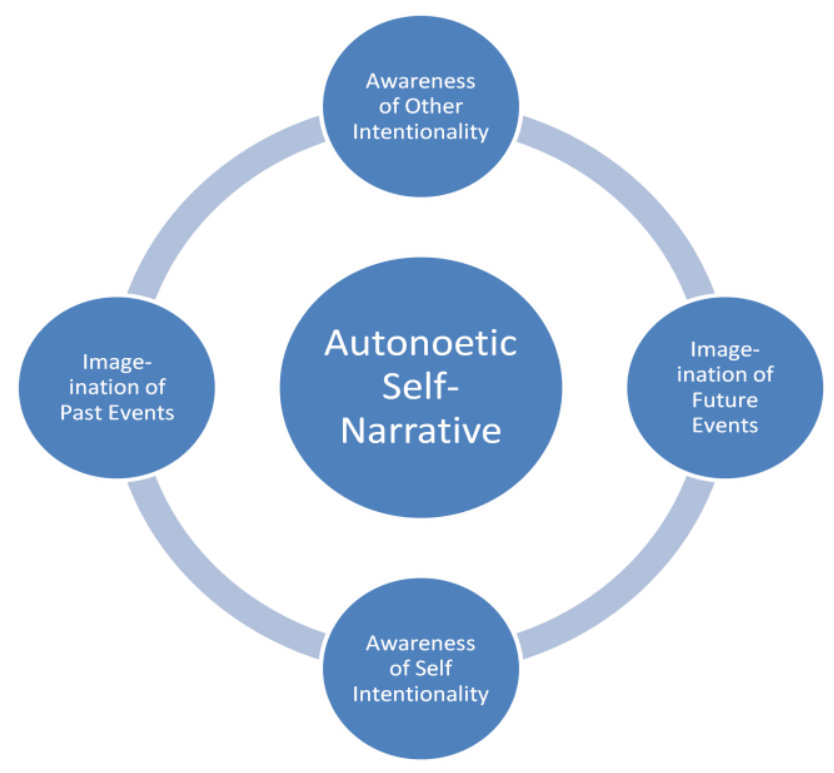

We humans understand our self-narratives, the narratives we create ourselves, the stories told to us by others, and the stories told by track-ways, by identifying with the characters. In other words we iteratively pretend to be the agents involved in the story in a turn-and-turnabout manner. These elsewhere-and-when agents include past and future imagined selves.

Clearly then, there could be no narrative faculty without the autonoetic self-narrative at its core. And without the narrative faculty there could be no elsewhere-and-when social competences (see below), which all require the projection of the autonoetic self into the 'minds-and-bodies' of elsewhere-and-when entities, including past and future selves. 


\section{The Cornerstone Cognitive Capacities Enabling Shared Intentionality/Cumulative Hominin Culture}

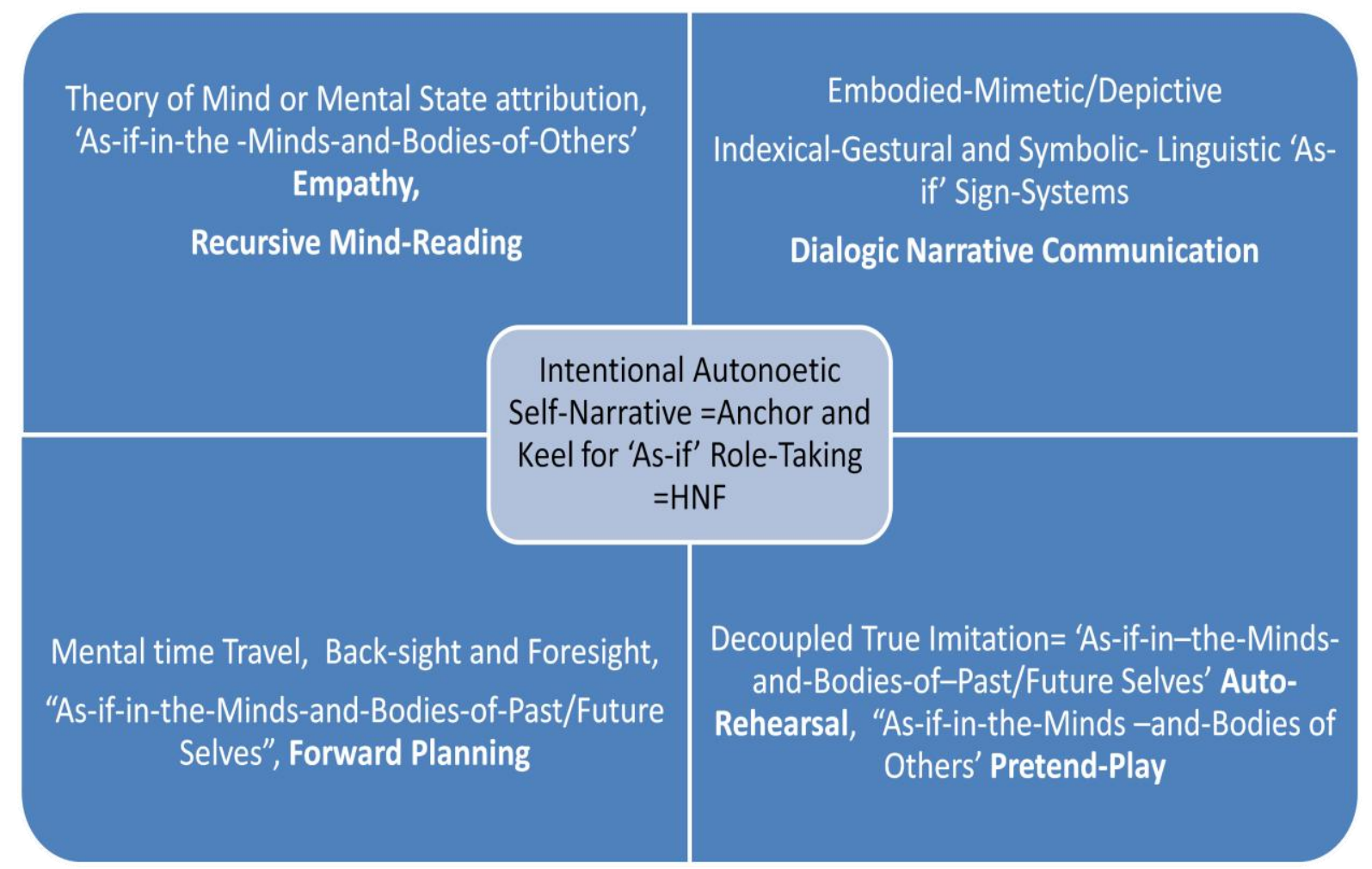

Of course, it is not really possible to separate autobiographical memories of one's past and future selves from the past and future selves of other entities. Our autobiographical memories and future narrative projections all have other significant agents involved. The hominin narrative mind is a social organ. That is why we cannot help anthropomorphizing or imputing intentionality to other animals when tracking and hunting.

This is also true when we are studying cognitive attributes of human toddlers and other animals. Hence many theorists cannot help giving apes and human toddlers the benefit of the doubt when it comes to their possibly exhibiting the elsewhere-and-when social competences of humans over the age of four. Lynda McNeil's otherwise brilliant paper (McNeil 1996) ${ }^{57}$ on

\footnotetext{
${ }^{57}$ She is the only other theorist I have discovered so far who directly views the cognitive phylogeny/ontogeny of all hominin culture and communication as being narrative. She calls us Homo inventans.
} 
the fundamental narrativity of hominin cognition and its phylogenetic and ontogenetic responsibility for the evolution of all hominin culture and communication is a good example ${ }^{58}$.

In sum, I hold the four capacities above are all made possible by the quintessential hominin cognitive trick of pretending that something is what it is not. That something must include the sense of an intentional autonoetic self, for one must be aware that one is pretending to do or be something else. Otherwise one is going to get 'lost' in narrative, pretending states of mind. For example, consider what happens when we have nightmares when asleep ${ }^{59}$. Here we arrive at an intellectual water-shed: many other cognitive theorists believe that the autonoetic selfnarrative could not have emerged in our cognitive evolution before linguistic communication [for example (Dennett 1991). I now want to turn this view thoroughly on its head.

\subsection{The 'Evolution of Language' Debate.}

The absolute necessity for the prior emergence of the autonoetic self-narrative is hardest for us to realize in the case of the cultural institution of language. It is so difficult that many theorists have believed that language is not a cultural invention: speech evolved somehow through some sort of 'magic cultural bullet' or 'monstrous genetic mutation', and only then could the self-narrative evolve. I believe the reason it is difficult is because our capacity for narratively mentalizing together or when alone is encapsulated, not open to introspection.

Therefore using the remarkable cognitive tool of language for communication, remembering, and thinking (we are doing it right now through the printed word) is almost completely automatized in the plastically developmental neural pathways of almost all modern human mind/brains. However, some us are born deaf and have to learn gestural sign languages: if there is no local cultural institution of sign-language, deaf children spontaneously invent their own gestural languages between themselves. ${ }^{60}$ This is highly significant, for such ontogeny

\footnotetext{
${ }^{58}$ For instance, she interprets the hide and chase games of young gorillas as pretend-play. But all young of higher mammals, especially predators play these games. And the branch-breaking of leading male bonobos when whole troops are migrating en masse to other parts of their territory is claimed to be intentional trailmarking. Again, branch-breaking is a universal chimp sign of aggression, and so it is far more likely to be a warning to other troops of bonobos strangers.

${ }^{59}$ An old Buddhist saying: "Man is the only animal that dreams while he is awake".

${ }^{60}$ These gestural languages are always structured SOV (Subject, Object, Verb or Action) rather than the SVO of English for instance. Everyone reverts to SOV when communicating in gesture. For the narrative mind, what is most important is who is doing what to whom: the characters or agents are imagined and described first, and then the action that occurs between them. Historical examples of the spontaneous emergence of sign languages (as in Nicaragua) have been viewed as evidence of an innate Chomskyian language-module.
} 
could be seen as recapitulating the phylogeny of all modes of hominin narrative communication.

I say that what is going on is that everyone in the same cultural group (a group of deaf children, say) in a sense agrees to pretend that an arbitrarily chosen gesture or symbolic spoken sound means or refers to a certain entity, state of being, (or another symbolic sign or set of symbolic signs). For everyone in that culture that symbol is that referred-to third entity or state of mind because they have communally agreed to pretend so. ${ }^{61}$ Toddlers are practicing this cognitive 'trick' when they begin to take part in object-pretence games with older children and adults. They are collectively pretending an object is something else entirely. But they are not yet pretending they themselves are being something else entirely. That comes later, sometime after the age of three. I will discuss this qualitative difference further in the next section.

To someone not 'in the game', just as with someone else born into another culture, the symbolic object, gesture or sound is meaningless. It remains so unless they decide to get into the mind-set of the culture and learn to pretend in the same ways they do, as when learning a second language. The older one gets the harder this is to do, precisely because the hominin brain is largely plastically developmental: it is much easier to learn a second language when young.

Footprints and track-ways are not symbolic or arbitrary: they are indexical or indicatory, referring directly to their 'authors', but they are often very decoupled from them in space and time. The Laetoli Prints are an extreme example. However, which animals are referred to by the footprints of the track-ways in one's local ecology have to be learnt when young, similarly to the language signs of the culture one is born into. This tracking cognition evolved long before the cognition needed to understand language symbols, but it is partly based on the same 'cognitive trick'.

Mimetic/gestural languages (Donald 1991),(Corballis 2003; Corballis 2010) must have been in place first for the cumulative culture to evolve that could invent linguistic language. I hold the natural sign system we call track-ways provided the cognitive template for those earlier forms of communication, which are still used for the sake of silence when hunting.

\footnotetext{
${ }^{61}$ Take the word "cool" for example: it became a descriptive musical term in jazz culture in the USA in the 1930 's. It has now come to mean a style of clothing or a certain state of mind. We are inventing new meanings for old words and inventing new words for new inventions or concepts (eg. post-modernism) all the time.
} 
Consequently there is still a direct connection between the two sign systems of language and track-ways.

Track-ways signs are real physical signs, faithfully or inexorably deictic, indicating or pointing to their referent. They are not arbitrary/conventional or symbolic, but I posit they were the only natural signs out there in the real world of our ancestors that could present a cognitive 'bridge' (or template) to the use of symbolic signs. That bridge was made up of at first iconic (more physically real or 'part-of') Oldowan mimetic or depictive communication, and then indexical (much less physically real or part-of) Acheulian gestural languages were invented as neural capacity for the narrative faculty expanded.

Linguistic communication is without a doubt the greatest invention of the eusocially narrative hominin mind, and it must have had a huge feedback effect on our self-narratives and our cultural institutions (which also have narrative structure). However, only already narrative hominin minds, able to communally pretend they were in other places, times, and other bodies and/or minds (and therefore able to systematically and speculatively read track-ways) could have communally invented language. This explains why linguistic communication had to come late, not early. One needs social, shared pretence, before one can have true words. In short, reference is the fiction of identity. ${ }^{62}$

\subsection{Overview of the HNF and the Cornerstone Competences}

To sum up: all four capacities made possible by the hominin narrative faculty therefore are at bottom self-aware. I mean self-aware in the sense that one must have a self that knows it is pretending in order to travel in time or be in someone else's mind, or pretend to be someone else or a future self when intentionally practicing any skill (such as learning a new language), or communally pretend with other selves that something is what it is not. Full-blown TWR requires the same faculty: imagining one's self as a cougar depends on self-representation, but it is not an instance of self-representation.

Remember here my metaphor of the autonoetic intentional self-narrative being like a steerable boat, in an elsewhere-and-when ocean of possible characters interacting in limitless ways in any place or time (including someone else's mind in the here-and-now). Therefore without possessing an up-to-date holistic self-narrative, no human being can function normally, or 'steer their life-way' through their social worlds, as various pathological mental

\footnotetext{
${ }^{62}$ This phrase is not mine.. It was presented to me as a 'slogan summary' by my supervisor Kim Sterelny, after reading a draft of this section on language evolution.
} 
states clearly reveal. In chronic schizophrenia, for example, when the sense of self-autonomy is lost, the mind gets taken over by imaginary entities. The sufferer is said to be 'not in the real world', because usually they break many of the social norms or rules of the culture they were born into.

The self-projection (=as-if role taking=emulation/imitation of absent entities) of pretend play is made possible by the past and future mental meta-representations of the autonoetic (=selfknowing) narrative that all normally functioning humans must have. It consists of an awareness of an intentional self travelling from the past to the future in company with the intentional selves of other hominins. This is the cognitive core of the HNF in my model, and makes the cornerstone social competences possible.

I repeat: this kind of self-referential as-if role-taking also occurs with agents being related to in the present. As Rakoscky (Rakoczy 2006) and Tomasello (2008) have pointed out, the shared intentionality of turn-and-turnabout, as-if role taking of children's pretend play, is social rehearsal for later taking up the roles required for all human social institutions to function. The point is, without an objective, first-person, autonoetic sense of self they could not know they were pretending.

Pretending to be in the minds-and-bodies of others is the cognitive basis of the hominin empathy/theory of mind (=mental state attribution=recursive mind-reading) competence. It also describes what is going on when we are telling each other (and listening to) our stories and/or making the collaborative plans for future action sequences that are behind all human culture. What is occurring between two or more agents in the here-and-now of conversations is shared intentionality, or communal projections of similar but separate selves into the future or the past.

I have explained my models of the HNF and the four cognitive competences as much as possible given the space available, and have stated there is a qualitative difference between the minds of apes, 2.5 year old children, and children aged between 3 and 4 . I argue this is when the HNF begins to fully function in humans, so now we need to look at the developmental and comparative evidence for this qualitative change. To relate back to TWR cognition before we do so, forgive me for reminding my readers one more time that all Kung San children know their mother's footprints from all others in the band by the age of four, and therefore must recognize their own. My point is that this could not occur unless their autonoetic self-narrative had begun to emerge. 


\subsection{The Qualitative Uniqueness of the Narrative Faculty.}

Apes are extremely good here-and-now behavior readers, as are humans, but only humans can share mental experiences that imagine the self and other selves interacting in the past or future. Tomasello (2008) calls these exchanges of communication (not just linguistic) about elsewhere-and-when agents and events "dialogic cognitive representations": this is the communication by which all culture is cumulatively transferred to peers and ensuing generations.

This transmission of culture cannot occur without what he calls 'the first and third person bird's-eye view': this is what the self-referential hominin narrative faculty provides. So adult human elsewhere-and-when social skills (the spin-off cornerstone competences) conferred by the HNF are qualitatively well beyond the here-and-now social skills of apes and 1.5 to 2.5 year old children.

In this section I present developmental and comparative evidence of the signature developmental manifestations of the core brain structure behind the uniquely hominin narrative faculty. One is external: children's turn-and-turnabout pretend play with peers that begins between the age of 3 and 4 . The other is internal: at the same age the 'fog' of childhood amnesia begins to fade as the child's self-narrative starts to take shape. In other words an autonoetic (self-knowing) meta-representation of an intentional self with certain desires and beliefs, travelling from the past into the future, starts to form in the child's mind. ${ }^{63}$

As Suddendorf (in Corballis and Lea 1999) says, between 3 and 4 is when the child begins to remember having personal (autonoetic) experiences at different past times. This makes Daniel Dennett's (1987) second-order intentionality possible. To reiterate: the child can know that it knows something another child doesn't know because the child can remember its own past experiences. She can remember/imagine her past-self being mentally and physically there at the time and in the spatial context of those experiences. Therefore she can know that another child, who was not present in that same place at that same past time, cannot know what she observed happening at that time.

That is why normal children only begin to pass false belief tests between the ages of three and four, and it is said that they have acquired TOM, or a theory of mind. However, most of

\footnotetext{
${ }^{63}$ By this age the brain is nearly full-grown and is consuming more glucose than it ever will again in the child's life-time.
} 
these tests are verbally presented. Recent preverbal TOM tests have been interpreted as showing that infants of roughly 1.5 years can read minds to some degree [(Baillargeon, Scott et al. 2010) for full review]. If true, and some theorists are skeptical (for example Sterelny pers. comm.), this indicates that TOM is developing earlier than the autobiographical self.

I am not skeptical at all, but neither am I impressed because I hold that infants are only exhibiting here-and-now TOM: they are better at reading the first-order intentionality of hereand now body-language than adult apes (but all higher animals can read body-language). I am not surprised that even infant humans are better behaviour-readers than adult apes, because hominins have probably always been able to hold longer sequences of actions than chimps in their minds.

This cognitive capacity was due to their free-handed capacity to carry things for long distances [(Ozvath and Gardenfors 2005), (Osvath and Gärdenfors 2007)]. Morphological changes indirectly bring about cognitive changes, because all cognition is inherently embodied. I have not the space to expand on this crucial idea here, but see Jeffares (2011 in press) for good review. So better here-and-now intentionality-reading was one of a package of necessary but not sufficient pre-adaptive traits inherited from the Ardipithecans, and it is only a quantitative difference from the ape mind-set.

I have already argued that the evolutionary window of opportunity for the earliest hominin capacity for elsewhere-and-when true imitation needed to read social track-ways narratives had to be the great-ape capacity for true here-and-now imitation. ${ }^{64}$ In the same vein, greatapes possess some here-and-now TOM (Tomasello 2008), and human toddlers have more here-and-now TOM, but only hominins over the age of three can have elsewhere-and-when TOM. Absentee conspecifics are out of mind to a here-and-now minded ape. They are not out of mind to an elsewhere-and-when minded hominin. I believe this is a qualitative cognitive difference from every other species on this planet, including chimps. The next section presents the developmental and comparative evidence that explains why I hold this view.

\footnotetext{
${ }^{64}$ Besides, like Suddendorf (1999) I do not think the HNF (he calls his model the "meta-mind") comes on suddenly: I think the way to view its emergence is that it very quickly 'comes to fruition'.
} 


\subsection{Developmental and Comparative Evidence of Ontogeny of the HNF.}

I wrote "Intentional Autonoetic Self-Narrative" in the centre of my model of the HNF. To fully imagine being in the minds-and-bodies of others one must have an awareness of others as being intentional beings like one's self; that is, having desires that they want to fulfil, and beliefs about how they can fulfil them. Here again some theorists may protest, because children as young as nine months relate to adult others as being intentional (Carpenter, Akhtar et al. 1998)(Tomasello and Rakoczy 2003).

These two theorists hold that this developmental stage (they call it the "9 month-old revolution") is the most significant change in human cognitive development. In other words, they see it as the unique social competence of the human mind. Further to this, they believe that it is joint-attentional exchanges with adults and older children, manifested as turn-andturn-about games using objects as symbols (pretence) and linguistic communication (pointing and naming at first, then introducing propositions that refer to the child's own mental states) in the ensuing years that creates a second stage of development, which they believe occurs between three and four.

I think 'joint attention' and 'pretence-with-older-others' behaviours that Tomasello and Rakoscy (2003) (Carpenter, Akhtar et al. 1998) describe in human children between 9 months and three years of age merely indicate even human infants might be better readers of hereand-now intentionality in others (reading their desires) than adult apes (so why not call it here-and-now TOM ?). Reading others' here-and-now intentionality or present desires (which includes the desire of older children and adults to pretend some object or some sound is something that it is not) is what infant here-and-now TOM consists of; it is Dennett's firstorder intentionality.

The underlying psychology of here-and-now TOM in both apes and human toddlers is emotional empathy and understanding body-language, which effects the here-and-now reading of someone else's desires. Just as I am not skeptical re evidence of here-and-now TOM in 1.5 year old toddlers, I am not skeptical (how could one be?) re evidence of 9 month old infants' reading of others' intentionality. But again I am not impressed.

Children younger than three or four (and adult apes) don't know they are reading others' intentions, or were reading them in the past; and that autonoesis or self-knowingness is what is required for second-order intentionality. Tomasello and Rakoscy think this kind of selfreflection is only acquired through language. I say this self-referentiality is neurally 
entrenched, and it became entrenched early in hominin evolution because hominins started reading their own old track-ways on an everyday in every way basis. I think the reverse is true: children can only begin to understand words used to describe desire/belief mental states of the self and others when they have begun to acquire a sense of an autonoetic self.

I hold that a sure sign that apes and toddlers are in the same here-and-now TOM, intentionality-reading, emulation/imitation 'boat' is they are both notoriously incapable of self-inhibition. Only with the elsewhere-and-when cognition of seeing one's self objectively as an intentional agent travelling through time can one understand that if one inhibits one's own desire for an immediate reward in the here-and-now, a future 'one' (a future self) will get a much better reward, or at least not get a negative reaction (from others or the environment) in the future. What is required is what Tomasello et al (2005) call the "first and third person point of view".

After the age of three children can be reasoned with on this level; before that age they cannot, and that is why parents speak of the "Terrible Twos". Later on they refer to the "Tiresome Threes": because children have acquired the elsewhere-and-when autonoetic narrative faculty, they continually ask "why is it (what is the reason) that I (first person) can't have it now?" "Because I say so" is very often the answer of the exhausted parent (third person), tired of trying to explain why. I agree with Tulving (2001): autonoesis is the key to the evolution of everything that is unique about hominin cognition, culture and communication.

So: apes are good behaviour-readers like toddlers and can read the intentionality of a hierarchical sequence of goal-directed actions performed by other apes in the here-and-now. This constitutes incipient anticipatory cognition, but only for brief episodes in the here-andnow. Suddendorf and Corballis (2007) have stated that apes might have a small amount episodic memory. I agree, and that is precisely why I prefer to use the word narrative for hominin cognitive capacities. I say it makes them good here-and-now imitators for a hereand-now reward, and somewhat anticipatory in social exchanges. But not nearly as good as toddlers, who are rewarded with delighted smiles when they synchronously imitate adults in a turn-and-turnabout manner.

Even human toddlers are able to have longer sequences of behaviour in mind than adult chimps. Therefore why wouldn't they have more here-and-now TOM (be much better intentionality readers) than adult chimps, emerging at a much younger age? What the emergence of the autonoetic sense of an intentional time-travelling self at the core of the 
HNF confers on 3 to 4 year old children is elsewhere-and-when TOM, or elsewhere-andwhen intentionality reading. They can now mentally imagine being in someone else's bodyand-mind in the past and the future because they can imagine being in their own body and mind at other times, experiencing what their desires and beliefs were or probably will be in the future. ${ }^{65}$ This is the unique social cognition that only hominins possess.

In sum then: toddlers can read intentionality or attribute desires to the minds of others, of course, better than adult chimps can. But like chimps they are just 'being' in the here-and now. They can also take part in the object-pretence games instigated by adults and children older than four, who give facial signals that define their actions as 'for fun' and not for real. They can logically understand the 'rules' of simple pretense games with objects because they are able to keep long action sequences in mind, but they do not spontaneously instigate object-pretence games on their own, or with peers of the same age, when adults or older children are not around. Tomasello and Rakoscy (2003) admit themselves that children aged 2.5 years old hardly ever spontaneously instigate pretence games with objects.

Note that this human developmental age is agreed by most cognitive theorists to be the cognitive ceiling for enculturated adult apes, who have only rarely been seen to exhibit what can be interpreted as pretence that an object is something else. Chimps know what other chimps have seen or not seen, and can tell whether a human is intentionally denying them access to food or is 'accidentally' dropping it (Tomasello and Rakoscy 2003, Tomasello et al 2005, Tomasello 2008). I think a lot of what is going on with preverbal 'false-belief' tests is about extremely good behaviour-reading by toddlers. However we have not the space here for any further discussion on this matter.

What I must add here though, to get us back on track (excuse the pun) is that I think this great-ape here-and-now TOM, just like great-ape here and-now imitation, is what made the shift from here-and-now imitational track-ways exploitation to elsewhere-and-when imagination of an absentee track-way-author's actions, and his or her mental states at the time of performing those actions, at all possible. As I have said, the absentee author was very often one's past self. These great ape social cognitive competences are the reason I think only some great-ape lineage could have fully entered the social TWR niche. The reason only our lineage entered it was because we got locked in to specializing in a bipedal wetland-foraging niche.

\footnotetext{
${ }^{65}$ This is why I am a simulation theorist when it comes to TOM development, not a 'theory-theorist'.
} 
I repeat: it is only after the age of three that children begin to indulge in the true 'as-if' roletaking of pretend-play, acting out narrative scenarios with one or two of their peers. They are also seen to 'slavishly' exactly imitate a demonstrator's physical actions towards a goal or reward, when adult chimps revert immediately to emulation if offered an easier route to the reward. For example, when 3 to 4 yr-old children were presented with a see-through perspex box that very obviously revealed the first two of the three manipulative moves (learnt beforehand when dealing with an identical but opaque wooden box) were completely unnecessary, they still repeated the first two actions ${ }^{66}$ in order to get the reward.

Adult chimps imitated the demonstrator's three manipulations faithfully without hesitation with the opaque box, but immediately 'cut to the chase' and made only the third manipulation when dealing with the clear box (Horner and Whiten 2005). Until recently there has been a consensus view (but see Whiten 2000) that apes cannot truly imitate a sequence of actions, they can only exhibit goal-directed emulation of the product of those actions. However the above and other recent experiments reveal that apes can and do truly imitate associatively in order to obtain a tangible reward in the here and now.

They will do so quickly and quite predictably, especially if the demonstrator is a trained conspecific. This bespeaks pretty good here-and-now social learning skills, and is an important feature of ape cognitive behaviour in itself, for it indicates they have the cognitive capacity for here-and now pragmatic choices between here-and-now emulation and imitation. So they are very good behaviour-readers and can make contingency plans in the here-andnow, but only for an attainable reward in the here-and-now. In terms of the here-and-now, then, they are very smart animals (and very competitive). The fact is however, apes never exhibit decoupled true imitation, such as occurs in human children's auto-rehearsal and pretend role-playing. Like toddlers, they are locked into here-and-now cognition.

Given the opaque/clear box experiment above, human children would appear to be dumber than apes in the here-and-now. However, the demonstrator was an adult, and three to four year children are inherently loathe to question an unknown adult's directions, especially in the unavoidably formal, 'teacher-ish' sociality of experimental situations. The question is, why this 'slavish' imitation. I think the answer is in their capacity for elsewhere-and-when cognition. Here is my mind-reading attempt re these children's mental states: "I wonder why

\footnotetext{
${ }^{66}$ I wonder if this experiment has been done with much younger children. I predict they too would 'cut straight to the chase' like adult chimps, if the presenter did not make play-faces, and was someone not much older than them, or better still, a puppet.
} 
he made those first two moves? Maybe there was another reason for making those dumblooking first two moves? Oh well, I'm still going to get the sweet anyway, and imitating adults pleases them".

Pretend-play sequences between post-four year olds become much more elaborate, with heated discussion of what the rules of the 'game' being played are, and who is going to take which role in the communally imagined narrative behind the 'game'. This is what Tomasello (et al 2005, 2008) calls "recursive role-playing". The narratives being acted out are based on mythic tales and adult behaviours exhibited in the cultures they are growing into (think of 'Cowboys and Indians' here, or 'Doctors and Nurses'). These pretend-play games are 'dressrehearsals' for later taking up roles in human cultural institutions (Rakoczy 2008).

Suddendorf's model of the "meta-mind" ( in Corballis and Lea, 1999) is very similar to my concept of the hominin narrative faculty and he also suggests that its emergence makes mental-time-travel, theory of mind, communication about past and future events and characters, pretend play and auto-rehearsal possible. He thinks it was phylogenetically in place by 1.5 mya, the beginning of Acheulian Culture, and holds that it ontogenetically begins to be manifested between the ages of 3 and 4, when the child begins to be able to mentally time-travel. As he says, "the child appears to enter into a new world", or (1994) "the fourth dimension". I say they begin to develop elsewhere-and-when cognition, based on the anchor, keel, and intentional steering-into-the-future capacity of an autonoetic self-narrative.

\subsection{Summary and Overview of Phylogeny and Ontogeny of Narrative Faculty.}

I have argued hominin elsewhere-and-when narrative imagination was well on its way to being a core faculty by 2.6 mya, the advent of Oldowan Culture, and had been incrementally evolving for around 1.5 million years before that date because our earliest ancestors had begun cognitively exploiting the track-ways made by band-members and themselves for safety and orienteering (for example re-finding rich foraging sites). The very new find of 3.4 mya cut-marked bones robustly supports this view (as does the 3.6 mya Laetoli dual adult track-way). The 3.5 mya pre-Oldowan mind/brain must have been already far more foresighted or cognitively elsewhere-and-when than that of our here-and-now ape cousins.

Even highly enculturated apes like Kanzi have never been seen to exhibit more than one or two instances that could be interpreted as pretend play. Young apes do play the rough and tumble and follow-the-leader games that the young of all mammalian predators indulge in, 
and can imitate their peers exactly in the here-and now, but never indulge in the decoupled true imitation of absent ('elsewhere-and-when in space and time') peers required for pretendplay. The imitation of elsewhere-and-when more skilled conspecifics demanded for autorehearsal of skills is also beyond them, which is why it takes them eleven years or so to learn how to crack nuts. Their social learning competences, while better than most higher mammals, are nowhere near as effective as those of humans.

I have argued this is because apes have no elsewhere-and-when narrative cognitive capacity to speak of. All they have is the anticipatory reading of goal-oriented peer behavior in the here-and-now required for exact imitation, some TOM/intentionality-reading, probably based mostly on brief moments of gaze-reading, for the sake of competitive deception, alliance negotiations, group hunting of colobus monkeys and other chimps, and so on. However, they are highly competitive, very smart mammals in the here-and-now, great body-language readers and so on. They are known, for instance, to exhibit some simple forms of intentional mimetic and gestural forms of here-and-now communication (McNeil 1996 for example).

I hold that human children under the age of three are somewhat similar here-and-now creatures, but much smarter behaviour-readers, and with far more TOM ability or first-order intentionality-reading in the here-and-now. It is perhaps far too easy for adult developmental psychology experimenters to impute cognitive capacities to them which have not developed yet, just as occurs with some primatologists and the trainers of highly enculturated apes. On the other hand, no creature on this planet is more directly enculturated (by being continually emotionally rewarded for playing synchronous reciprocal imitation games) than human children. Our mind-brains are culturally developed more than any other creature on this planet.

We should not forget this fact when discussing whether children can 'truly pretend' before the age of three. I think they understand pretence sequences with objects in the here-and-now, but do not have the meta-representation of an autonoetic self that is pretending to be someone else until somewhere between three and the age of four. There is evidence that even twoweek-old infant hominins are good here-and-now imitators ${ }^{67}$ : in fact some imitation theorists would say we are imitators by default (Meltzoff and Decety 2003). The idea of humans being imitators by default makes perfect sense to me, because we are narrative-minded by default.

\footnotetext{
${ }^{67}$ There is lots of controversy around these claims, but we have not the space here to enter the fray.
} 
So I agree with them ${ }^{68}$, with the proviso that once the HNF was in place and co-operative culture could begin, there would have been inexorable positive feedback for excellent hereand-now and elsewhere-and-when true imitation abilities, in order for that culture to be transmitted to ensuing generations in a cumulative manner. The faster an adaptive cultural innovation can move through a population, the more likely that population is to out-compete other conspecific populations, so powerful group-selection forces would have led to these entrenched and therefore default imitation abilities.

I am thinking here of a very early emergence of group rituals of synchronous mimetic dancing and chanting (since some chimp troupes dance when it rains-Whiten 2000), and much later on group reward/punishment rituals, marriage contracts, and other social norms, not just the control of fire, the spread of linguistic languages, and so on and so forth. Let us look at some evidence from neuroscience once more.

I repeat: an early enlargement and widening of the more medial/posterior parts of the brain (temporal/parietal area) is definitely seen in the very earliest (2.3 mya) habilis endocasts (Tobias 1987). Neuroscience has proved these areas are part of the core brain structure that supports the default-mode as-if role playing, self-projecting 'episodic' faculty behind the four uniquely hominin social cognitive competences (Buckner 2007; Buckner and Carroll 2007; Schacter, Addis et al. 2007). To quote the neuroscientists Buckner and Carroll here:

"When thinking about the future or the upcoming actions of another person, we mentally project ourselves into that situation. Accumulating data suggest that envisioning the future (prospection), remembering the past, conceiving the viewpoint of others (theory of mind) and possibly some forms of navigation reflect the workings of the same core brain network. These abilities emerge at a similar age and share a common functional anatomy that includes frontal and medial temporal systems that are traditionally associated with planning, episodic ${ }^{69}$ memory and default (passive) cognitive states....these abilities, most often studied as distinct, rely on a common set of processes by which past experiences are used adaptively to imagine perspectives and events beyond those that emerge from the immediate environment" (abstract,p49)

\footnotetext{
${ }^{68} \mathrm{I}$ think this imitation by default idea is extremely important when it comes to certain aspects of human culture, like meme-exchanges and so on. We have not the space here, but I would like to say this: for me the idea of default imitation helps to explain why humans can be so clever and so abysmally stupid at times (especially en masse). Only humans could be 'brain-washed' into becoming teenage suicide bombers, or help think up plans for efficiently exterminating 6 million other humans.

${ }^{69}$ I prefer narrative: after all an episode is merely the shortest possible narrative conceivable.
} 
Therefore how the autonoetic autobiographical self-narrative could have evolved is the definitive question re the nitty-gritty of our cognitive evolution (Tulving 2001). I firmly believe my thesis and in particular this chapter has provided a very plausible answer to that question: through a couple of million years or so of reading the stories told by one's own old track-ways.

Right at the beginning of our evolution simple social track-ways exploitation, consisting of continually stepping in one's own track-ways (as well as those of others) for the sake of safety and orienteering, triggered the evolution of this autonoetic narrative sense of self, which conferred second-order intentionality and a mental awareness of others as separate but similarly intentional selves on the early hominin mind. Thereafter the narrative faculty and hominin elsewhere-and-when social skills continued to co-evolve through selection for better social TWR skills, due to inexorable increases in socio-ecological complexity. My 'triggering track-ways theory' is therefore a thoroughly orthodox 'social brain hypothesis'.

Once the HNF and the cornerstone competences were somewhat entrenched, adaptive cumulative co-operative culture could begin, when triggered by environmental contingencies. Thereafter positive feed-back into the genetic/cultural co-evolution of the hominin narrative capacity for elsewhere-and-when social competences would have been inexorable. These elsewhere-and-when social competences, enabled by self-conscious or autonoetic shared intentionality (Tulving 2001, Tomasello 2008) created cumulative, collaborative eusocial hominin culture and communication. These emerging competences were also what made fullblown systematic and speculative extractive-foraging TWR skills in the extractive foraging domain possible.

It was entering the simplest, here-and-now cognitive niche of imitation of conspecific trackways for safety and orienteering in the first place, riding on the back of other attributes conferred by great-ape here-and-now social competences and a semi-aquatic bipedal socioecological niche, that made the evolution of hominin culture, and communication of that culture, both horizontally and vertically, at all possible. In the next and final chapter I point out the major milestones on the hominin co-evolutionary road towards linguistic language, Homo sapiens, and behavioural modernity. We will find that most of these milestones, especially the earlier ones, were due to selective forcing by dramatic environmental changes. 


\section{Chapter Six: The Hominin Evolutionary Trajectory.}

\subsection{The Environmental Forcing of Hominin Evolution.}

My chronological story in this final chapter is mainly about how volcanism and earthquakes or faulting iteratively and sometimes drastically adversely affected the wetlands on which the earliest hominin locals were depending for their highly nutritious staple diet. The Rift was extremely volatile throughout the Late Pliocene and Early Pleistocene because it had (and still has) a thinner mantle or lithosphere overlying the earth's molten inner layer than any other land mass on earth.

Volcanic ash is highly permeable to water, so wherever it is laid down in thick layers on flat land aridification immediately ensues. Ash-covered flatland areas that had been prime farming land in Mexico were still deserts fifty years later after a local eruption in the 1940's. Basalt lava flows re-vegetate far more quickly because they retain water, but molten lava tends to run downhill before it cools and solidifies into basalt. Therefore lava flows can dam even very large rivers completely from one week to the next, or at least make them suddenly impassable for the next season's spawning fish runs.

Alluvial action quickly washes ash into all lower lying basins and lacustrine swampy areas. This is why fossil deposits are so often associated with volcanic events: volcanic ash sedimentation quickly covers up bones before they disintegrate due to exposure to the elements, or can get eaten by scavengers. Volcanic ash chemically preserves bone. It also affects water chemistry drastically and suffocates all standing water biota in shallow lakes and ponds.

The huge amounts of carbon thus released turn them immediately eutrophic and conditions turn poisonous and anaerobic (no oxygen in water for aquatic fauna). This happened to several small lakes close to the epicentre of the recent Mt St. Helens eruption in the USA. Steeper more energetically alluvial areas are the first areas to recover, because ash is quickly washed away down-stream.

Large basalt depositions, as occurred to the east of the Turkana basin, weigh down the lithosphere and cause massive faulting and subsidence in nearby areas. All in all the "cradle of humanity" in East Africa was an extremely tempestuous environment to evolve in. Therefore my thesis is fully supported by the 'environmental variability hypothesis' (Potts 1996). Environmental change triggered the advent of Oldowan Culture and the 
encephalization that led to two new hominin species: the earlier habilenes and later erectus hominins.

It is also true that extreme glaciations occurred in the Northern Hemisphere not long (geologically-speaking) after the 2.8 polar reversal (Behrensmeyer, Todd et al. 1997) [(Thierens, Pirlet et al.) in press]. After an initial era of extremely wet climate, aridification in the form of extreme seasonality became more intense from 2.6mya onwards and peaked at 1.8 mya $^{70}$. Major faunal turnovers occurred in in the Turkana Basin (Hernández Fernández and Vrba 2006).

Areas of savannah expanded throughout the Rift. I think thick layers of volcanic ash would have iteratively hastened and intensified these effects (as in our Mexican example above). These global and local environmental changes working together would have played havoc with the wetland environments preferred by the pre-Oldowans.

I have pre-empted the chronological narrative structure of this chapter somewhat by mentioning the adaptive plateau of the socio-ecological niche of the Ardipithecans between 5.8 and 4.4 mya in my introductory section to chapter one. However we will begin by returning there in the next section because I think the evolution of obligate bipedalism was caused by a truly massive volcanic event, also mentioned in that section. Therefore this event is the right place in time for our evolutionary chronicle to begin.

\subsection{Leaving the Trees Behind.}

At 4.39 mya a truly enormous volcanic eruption occurred in the Ethiopian Highlands. A thousand cubic kilometres of volcanic ash are estimated to have been deposited as far as the Sea of Aden to the north of the Rift. Ash was deposited at an average height of 1.7 meters, and at this depth all standing forest is destroyed [WoldeGabriel et al in (McCoy and Heiken 2000)]. The Awash Basin in the Northern Rift, where the 4.4 mya Ardipithecan fossils were found, became a huge open fluvial area of lakes and wetlands.

An extremely large lake (7000 sq.km) was also formed in the Central Rift. This occurred because the major river that drained the Ethiopian Highlands south into the Turkana Basin, and then ran from the north-east of the Basin to the Indian Ocean, was blocked by massive lava flows and subsequent faulting. Around 3.95 mya this river (the Omo) found another

\footnotetext{
${ }^{70}$ It is interesting that his period of $800 \mathrm{ky}$ of glaciation effects on climate equates well with the period between the next polar reversal at .78 mya and the beginning of the Holocene $20 \mathrm{ky}$ ago.
} 
channel from the south-east corner of the Basin to the Indian Ocean and this huge lake was quite suddenly drained.

A vast expanse of open fluvial wetlands would have been left behind in the Turkana Basin, creating innumerable spawning grounds accessible to huge runs of fish from the Indian Ocean. I think these combined dramatic environmental changes forced some local Rift populations of Ardipithecus from their adaptive plateau of facultative bipedalism, nesting in trees at night, and foraging in flood plains and wetlands within upland closed forests during the day.

Our evolutionary narrative begins here because this is when hominins 'left the trees behind' and became fully terrestrial and bipedal. They therefore had to enter the social TWR niche for safety and in order to navigate through vast expanses of open, feature-less upland wetland environments. However it probably did not require much beyond social TWR for the orienteering needed to procure fish, shellfish, bird's eggs and fledglings, amphibians, reptiles and micro-mammals such as cane rats. To use an ancient metaphor here: the pre-Oldowan hominins were living in the 'Garden of Eden'.

Around 2.8 mya a second massive peak in volcanic activity almost equal to the 4.4 mya eruption occurred, just after the Matayuma-Gausse north/south reversal in the polarity of the earth's magnetic field (WoldeGabriel in McCoy and Haikin 2000). Such reversals cause before-and-after peaks in volcanic and earthquake activity, and extreme glaciations begin. ${ }^{71}$ Climate after 2.8 appears to have become much cooler and wetter for a short period (geologically-speaking). Lakes in the Rift did become very large just afterwards.

The earliest Oldowan sites (Gona, 2.6 mya, Bouri 2.5) were in low-energy fluvial wetlands around large lakes in the Awash River drainage basin in the Northern Rift (Quade, Levin et al. 2004). These vast wetlands had been the normal topography there ever since 4.4 mya. A recent analysis of carbon isotopes in a large sample of fossil herbivore teeth dating from the mid to Late Pliocene (4.4 to 2.4 Ma) indicates a heterogeneous mosaic of wetlands and closed (probably riparian) and open woodland areas (montane deciduous trees) that suited browsers, far more than obligate grass-eaters. Thereafter there was some increase in savannah, but not as pronounced as that which occurred after the next polar reversal at .78 mya. Therefore my

\footnotetext{
${ }^{71}$ There was another major volcanic event that deposited deep layers of ash as far as the Sea of Aden at the next reversal dated .78 mya (called Gausse-Brunhe). It also caused extreme glacial cycles that carried on throughout the rest of the Pleistocene.
} 
post-2.6 mya Oldowan scenario has far more in common with the 'environmental variability hypothesis' (Richard Potts 1996) than the traditional 'forced out onto the savannah' model re environmental triggers for the encephalization that led to the first true hominins.

In sum then, extreme global climactic effects and a peak in volcanism and earthquake activity causing catastrophic changes in the environment did occur at 2.8 Ma. Their combined effects were centred in the East African Rift, the 'ancestral cradle' of our hominin forbearers. The result was that some hominin populations were deprived of their lacustrine 'Garden of Eden' for shorter or longer periods. They had to culturally adapt by habitually applying their narrative anticipatory cognition to new extractive foraging techniques whenever their local wetland resources were destroyed: the acquisition and lithic butchery of large carcasses. I think this why continuous Oldowan Culture began: social TWR skills and the HNF had to be applied to consistently hunting large mammals.

\subsection{Leaving the 'Garden of Eden'.}

I think this metaphor is warranted because I have hunted, fished and gathered in all types of environment. The richest and easiest terrain to hunt and gather in is lacustrine and/or estuarine wetlands, simply because such environments are full of easily procured aquatic and semi-aquatic biota, and almost all terrestrial life needs regular access to fresh water. Hence all the first sedentary and more complex pre-modern human 'civilizations' began in such environments during the Holocene: simply because they could, due to resource-richness.

In the case of the East-African Rift its resource-richness was basally due to vast numbers of several species of large (up to 40 kilos) fatty cat-fish (besides eels and several other species of fish) that migrated from large inland lakes and/or the Indian Ocean (or the Sea of Aden to the north) up large rivers to spawn in the wet season in seasonally flooded wetlands (Cunnane and Stewart 2010). They were then trapped in ever-shrinking ponds and swamps as the dry season approached. They would have been easily captured by hand and/or with simple spears in such conditions. They are still captured by hand in shrinking river pools (see next page).

Add to this incredibly rich food source innumerable young and eggs of water-fowl, several species of very large fresh-water shellfish, countless numbers of frogs, turtles and small reptiles, sweet tasting easily obtained bulbs of swamp sedges, and plants like water-cress, thriving all-year-round on the edges of permanent water-ways in a tropical climate, and you will begin to understand why such an environment would be so attractive to our earliest ancestors. 


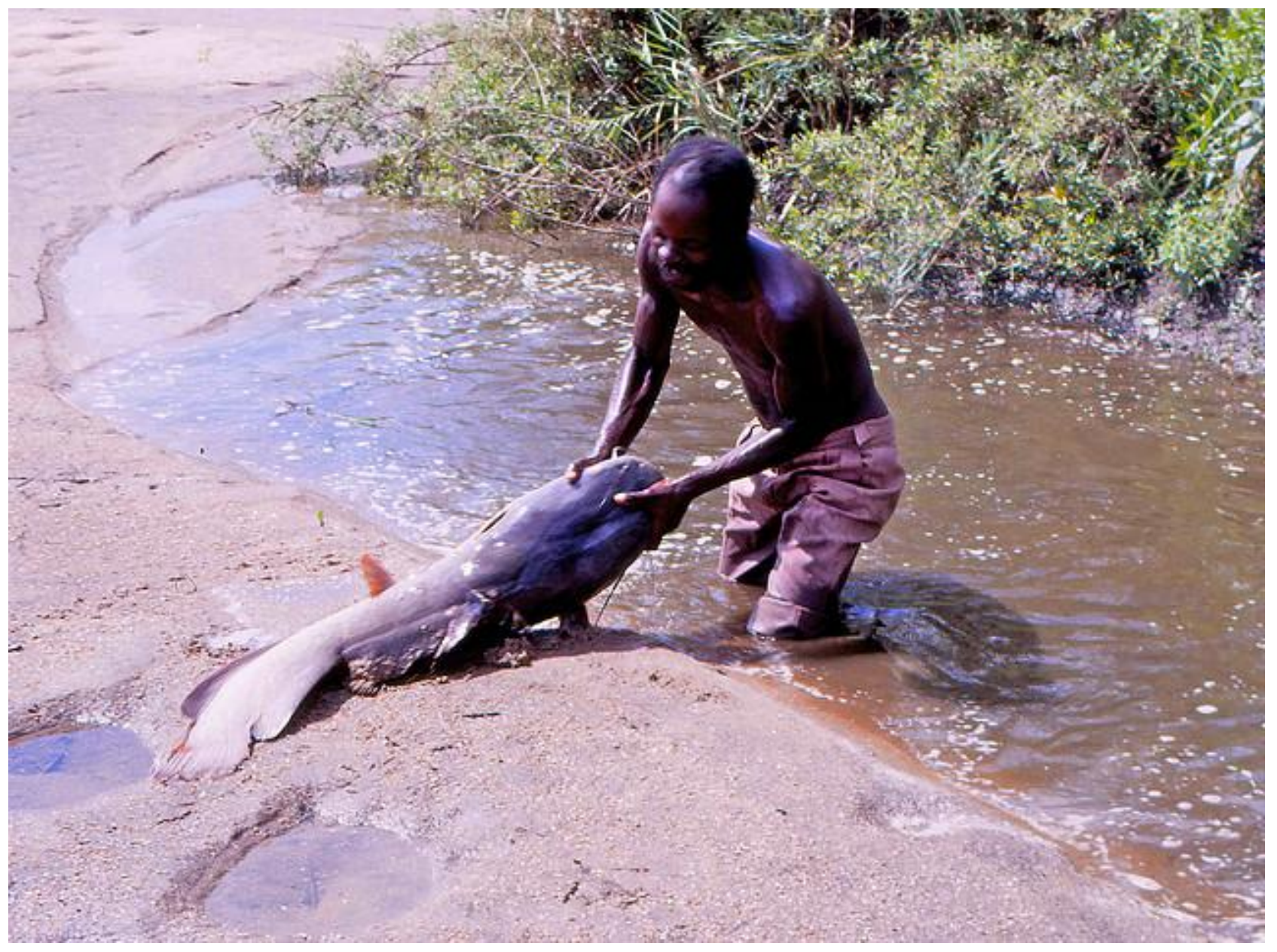

A large catfish caught by hand in small pool in a shrinking water-course (from flickr, a photo-sharing website)

Hominins were fully bipedal by at least 3.6 mys ago, so had a precision grip plus the morphological ability to throw stones very forcefully and accurately at any predator who tried to bother them. Since they could also run, wade and swim through shallow water (thus hiding their scent from land-based predators) faster than any four-legged pursuer, we should be able to fully understand my use of the 'Eden" metaphor.

Earthquakes and lava flows can completely block or change the course of rivers overnight, making upstream spawning grounds inaccessible to fish. It takes years for a population to recover from such devastation, for whole populations can be wiped out when long established 'home' spawning rivers become instantly impassable. This happened on the Frazer River (the major river in the province of B.C., Canada) in the 1950's when I was a child.

A gorge known as Hell's Gate became impassable just before that year's salmon run could get through due to a botched road-works explosion, but any earthquake or lava flow could 
have exactly the same effect. It remained that way for four years before human intervention created a fish-ladder for their passage. Salmon only live for four years, so the normal population was almost completely wiped out. It took more than a decade of assisted breeding and re-stocking from salmon from other rivers to get the population of returning salmon back to anywhere near what it had been before this catastrophe.

Meanwhile, local indigenous tribes that had depended on those salmon supplying their main food source for thousands of years, and in historical times their only cash income as well, were completely destitute and had to be supported by social welfare. In prehistory they would have been forced to migrate or starve. All kinds of animals exploit the 'windfall' of millions of dead and dying salmon in the early autumn, in order to build up fat for the winter, so they would not be present to be exploited either if the stock of salmon returning to a certain river valley was suddenly destroyed.

In East Africa catfish trapped in pools on flood plains left by desiccation at the beginning of the dry season would also be a wind-fall for all other creatures, so I argue for a similar scenario. Therefore loss of such basal resources could well have been ecologically catastrophic for hominin locals (as well as other animal populations). Thus an environmentally forced nomadic scenario would have been a common occurrence given the environmental effects of even quite minor earthquakes and volcanoes. So any cognitive ability to plan ahead, in order to culturally adapt to novel territories and/or co-operate with (or confront) strangers would have been a major asset to our behavioural repertoire, and would have been strongly selected for throughout the Plio/Pleistocene.

In sum, I argue that after around 2.6 mya environmental catastrophes were dramatically destructive to the wetland daily and seasonal socio-ecological niche of local hominins. They began tracking down large mammals and systematically manufacturing and transporting sharp-edged stone tools to carve them not just because they could, which is the scenario posited by many other theorists (Ovsmath and Gardenfors 2005 for example). They had to do so to survive.

In support of this environmental forcing of habitual hunting of large mammals scenario, I here present corroborating evidence from the Turkana Basin. This is where the first habilene (2.3 mya) and the first erectus fossils (1.8 mya) were found. There are numerous stone tool and bone sites throughout the Basin dated between 2.2 to 1.8 mya. Between 2.2 and 2.0 mya the Omo River outlet from the SE corner of the Basin was intermittently closed off by lava 
flows. These lava flows caused extensive faulting as well, resulting in over-all subsidence of the Basin (Bruhn et al 2011).

Finally the outlet to the Indian Ocean was closed off completely. A moderately large lake formed in the Turkana Basin, whose size fluctuated wildly with seasonal climactic conditions. A $300 \mathrm{~km}$ long mosaic of fluvial wetlands and floodplain riparian forest (much like the Okavanga Delta is now) along the Omo River drainage system, which would have been spawning grounds for huge numbers of fish, was cut off from lakes to the southeast and the Indian Ocean. Before 2.2 mya the Turkana Basin would have been a refugia for hominin wetland foragers; after 2.2 mya it was not. Further evidence of environmental mayhem is provided by the fact that the most dramatic faunal turn-over in the Basin's history occurred between 2.2 and 2.0 mya. (Bobe and Behrensmeyer 2002, Fernandez and Vrba 2006).

Close to epicenters of volcanism large numbers of macrofauna are immediately killed as well as all smaller fauna (large numbers of elk near the Mt. St. Helens volcanic eruption died due to volcanic effects). Further away they weaken from starvation (these elk were restricted to a nature reserve) and die more slowly. So there also would have been a window of opportunity as well as the dire necessity for some populations of 2.5 mya Awash Valley hominins and 2.2 mya Turkana Basin hominins to find these vulnerable targets using simple TWR. Volcanic ash is a perfect substrate for recording foot-prints.

They began continually carving the meat from large carcasses with sharp stone flakes, pounding the meat ${ }^{72}$ to tenderize it (Lieberberg 2009), and smashing the larger bones and skulls with rocks to obtain marrow and brains. I repeat: marrow and brains are the only other food source besides shellfish and fish that can supply large quantities of the fatty acids (lipids) necessary for healthy hominin brain growth (Crawford 1992, Broadhurst et al 1998, 2002).

This model of environmental change between 2.6 and 1.8 mya as a spur for an abrupt beginning to new foraging applications of simple TWR and the HNF explains the rather sudden appearance of habitual large carcass exploitation and the establishment of continuous Oldowan lithic technology (Plummer 2004). Further task analysis reveals the following: large animals are impossible for fangless, clawless hominin primates to carve or exploit in any way

\footnotetext{
${ }^{72}$ This explains the large number of spheroid stones found intermingled with flakes and cores at Oldowan sites.
} 
without cutting tools, and butchering them ${ }^{73}$ requires co-operative intentional behaviours between, at the very least, two individuals. Several more would be needed for co-operative transport and defence of the meat and defending such large amounts of it from scavengers (Rose and Marshall 1996 for review).

It does not matter whether the carcass was obtained by confrontational or passive scavenging or chased by a group of rock-throwing hominins till it was mired in a swamp and was clubbed to death. That said, as I argued in chapter three, the phylogenetic age of the human tape worm (1.7 mya) indicates that hominins were probably not habitually scavenging until around that date (and I posited that they were probably pirating the kills of wild dogs by then).

Co-operative food-sharing behaviours may well have already arisen in pre-Oldowan populations, as well as simple group hunting techniques used for capturing large numbers of small wetland animals. What I must emphasize here is that cognitive foraging adaptations can be co-opted for social interactions and vice versa. Increasing capacity for back-sight and foresight would make reciprocal altruism, more co-operative breeding, division of labour, intentional sharing and punishment of cheaters possible. Social punishment techniques such as group stone-throwing for control of in-group bullies and cheaters (Bingham 2000), and defence from predators and other hominin bands, could already have been a social feature by the advent of Oldowan hominin life-ways.

It is true that we have no paleo-archaeological evidence for this speculative scenario of continual co-operative culture made possible by the HNF until Oldowan Culture at 2.6 Ma. What we do have now though is the very recent find of cut-marked bones at 3.4 mya (McPherron et al 2010). This evidence shows some hominins were able to carve large animals a million hears before Oldowan Culture emerged. So why did they wait till 2.6 mya to start carving them often enough to form cumulative culture? When added to the incontrovertible evidence of a diet of wetland fauna (including crocodiles) at one site dated 1.95 mya (Braun et al 2010), my story that some populations of hominins began hunting large mammals because their normal wetland resources were destroyed becomes very plausible indeed.

\footnotetext{
${ }^{73}$ I can testify that butchering a large beast quickly (but carefully so there is less waste) with a steel knife is both an art and a science, and takes a while to learn.
} 


\subsection{The Advent of Oldowan Culture and the 'Co-operative Explosion'.}

Like Tomasello (2008) and Ozmath and Gardenfors (2005), I think the major anomaly in the narrative of hominin evolution that needs to be explained is the abrupt appearance and ongoing continuity of the Oldowan level of knapping expertise and stone resource acquisition and transport. Even the earliest sites of all (2.6 my at Gona and 2.5 mya at Bouri) show evidence of foresight, or anticipatory cognition (Semaw 2000). These sites reveal stone resource transport, knowledge of structural qualities and durability of different types of stone, quite complex knapping techniques and so on. Sometimes the nearest suitable stone resource was 10 to 13 kms away (Osvmath and Gardenfors 2005, Plummer 2004, Toth and Schick 2009).

Toth and Schick (2009) have carried out an experiment comparing stone knapping results from enculturated bonobos, first time human learners, and skilled stone-knappers. The results show that ape knapping is nowhere near as planned as Oldowan samples and modern results, or those tools made by first-timers. However Toth and Schick, like other continuity theorists, think our ancestors suddenly started making tools as functional as Oldowan tools while in the same socio-ecological niche as apes. I disagree, and hold that such evidence of planning abilities indicates that the HNF was quite well entrenched by 2.6 mya.

The knapping expertise exhibited by Oldowan tools and the 3.4 mya evidence of cut-marked bones indicates pre-Oldowan hominins had been making sharp stone edges for a million years. They would have been useful items to carry around, useful for sharpening simple spears and for hacking up very large catfish and sharing them where they were caught. Their bipedal wading and carrying capacities, precision-grip hands, anticipatory transport of sharpened stones and co-operative use of simple spears, clubs and stones were the only defensive and foraging tools required in their wetlands 'Garden of Eden'.

I am assuming, as do Toth and Schick (2009), that they had spears and digging sticks because we know one troupe of chimps makes very simple spears for impaling bush-babies in hollow trees (Pruetz and Bertolani 2007). These chimps (females in this case) sharpen the ends of their spears with their teeth. The very earliest pre-Oldowan hominins could have used sharp smashed-stone edges to sharpen their simple spears; and this would have developed into more controlled stone-knapping as the HNF came on line.

In sum then, the sudden appearance of Oldowan lithic application and expertise does indicate a recently forced change in life-ways, as well as the foresight and planning plus intentional 
co-operation made possible by the HNF and the cornerstone cognitive competences. Task analysis indicates these cognitive capacities must have been already somewhat in place in the hominin mind/brain in order to create such remarkable cultural answers to problems instigated by contingent environmental changes. So 2.6 mya is where the gene-culture/niche construction co-evolutionary 'wheel of fortune' begins to spin in earnest in our evolutionary trajectory.

The results were dramatic, resulting in two fully hominin sympatric species, Homo habilis and erectus, within half a million years, whose brains had doubled in volume relative to adult size when compared to chimps, ardipithecans, and australopithecines. They were sympatric species in the Turkana Basin: habilis survived there until 1.44 mya (Spoor et al 2007). Cortical expansion occurred in the parietial/temporal areas which are dedicated to social competences, and the medial prefrontal cortex, which we know is dedicated to planning of hierarchically arranged action sequences in all modalities.

Individual and group selection for expansion of neo-cortical neural plasticity continued to be triggered by extreme environmental changes throughout the Plio/Pleistocene and was scaffolded by positive feed-back from increasing TWR skill and cumulative social cooperation (needed to supply high-quality nutrients for development of an ever-increasing brain). The neo-cortex continued to incrementally expand during the Pleistocene as more application of the anticipatory cognition conferred by the HNF and systematic TWR skills to extractive foraging techniques was required.

I think social contracts such as long-term mating coalitions (Deacon 1997), (Ovsmath and Gardenfors 2005) and pedagogical teacher-pupil relationships using pretend-play mimetic communication would have been in place. Group-grooming rituals combining synchronous mimetic dancing and prosodic chanting would have been the socio-psychological expression of Oldowan co-operative culture.

Habilis, with their somewhat larger brains (2x the ape brain relative to body size) first appear around 2.3 Ma; erectus, with a brain doubled in size and a fully humanoid morphology (smaller gut, robust endurance-running human morphology and life history) appears around 1.8 Ma. In my chronological model we are approaching the end of the mimetic Oldowan Era. By 1.6 Ma true hominins had reached N.W. China, and more dexterous Acheulian hand-axe technology had emerged in Africa and Eurasia. I think the era of gestural language had begun. 


\subsection{The Gestural Acheulian Era}

A very short track-way of erectus fossilized foot-prints has recently been found at Ileret in the Turkana Basin((Bennett, Harris et al. 2009), dated 1.5 Ma (see their illustrations of one of the prints next page). One theory these foot-prints have brought consensus to is that erectus was definitely a long-distance, wide-ranging endurance runner by 1.5 mya, because the prints prove erectus had a fully human high inner arch. Some theorists also think it is fully human because there is no gap between the big toe and the second toe

Habitually unshod feet leave prints like the Laeotili Fossil Footprints and the modern Machiguenga footprint (section 4.2). There was undoubtedly a bigger gap between the big toe and the second toe in those prints than in the print we see below. However, when you look at the illustration of the same print on the next page, one can see why this happened. The depth of the print shows its author was stepping into a deep soft substrate. This would have naturally forced the big toe inwards as the front of the foot levered itself forwards out of such a deep substrate.

I mention this partly because Bennett and some other theorists think the slightly more splayed big toe of the Laetoli Prints indicates they were made by australopithecines. I disagree, and would suggest the following: whenever scientists find fossilised footprints like these, they should get immediately get expert trackers to have a good look at them in situ. It was the wildlife photographer and tracker Alan Root who realized the adult Laetoli track-way was a dual track-way, but he was shown them a year after they were found. Full-blown TWR is an art and a science. 

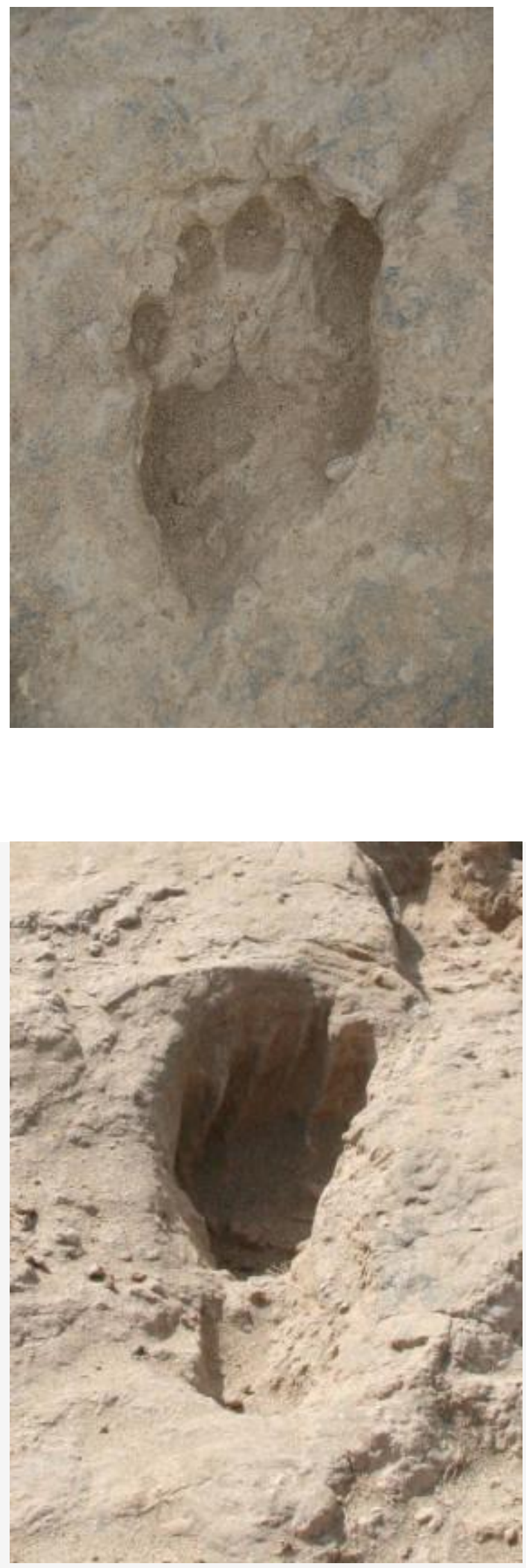
However, I do agree with Bennett and his colleagues that this footprint indicates a much less flat-footed morphology than that of the Laetoli hominins. Hence this footprint (one of several in a short track-way, but there were no illustrations of the other prints presented) and the robust physique indicated by the famous Turkana Boy skeleton (dated 1.6 mya) makes endurance hunting a pretty sure bet as being a mainstay hunting technique. We know this technique demands systematic TWR, because an individual animal has to be repeatedly tracked down (Liebenberg 2008). As I made clear in chapter two, systematic tracking requires good narrative imagination-not as much as speculative, but still a fair amount.

Erectus had colonized all warmer parts of the Old World by 1.6 mya. Migrating 'seedpopulations' would have had to learn the folk biology of new ecologies. Knowledge of animal behaviour is more important than technology. The reason I have made this observation and presented the footprint evidence for endurance hunting and systematic tracking above is to somewhat counteract theoretical assumptions that there was a hiatus in hominin cognitive evolution because Oldowan and Acheulian stone-tool technology both lasted for a million years. Like Suddendorf (Corballis and Lea 1999) I think what is basally unique about human cognition was fully entrenched by the beginning of the Acheulian Era at 1.6 mya.

I posit it marked the beginning of a cultural era of gestural language, the kind of communication that is still used for the sake of silence when hunting. The dexterity needed for gestural languages would co-evolve with the dexterity needed for hand-axe production and perhaps some simple knotting and plaiting technology. A fully developed HG life-style incorporating habitual fire control, grinding of nuts to make nut-cakes, and regular exploitation of elephant, fallow deer and gazelle (as well as aquatic fauna from the lake nearby) was in place at the now famous Gesher Benot Ya'aqov (GBY) site by the Early/Early to Mid-Pleistocene (Goren-Inbar, Feibel et al. 2000).

This paper dates fire control at $790 \mathrm{kya}$. Another equally complex site has just been discovered in northeast England, similarly dated. Goren-Inbar (2011) states that "all archaeological levels show fire-control", but gives no actual dating besides "Early Pleistocene". These fire-based completely HG cultures could have existed not long after our 1.5 mya erectus footprint was made. There is some evidence of fire use dated 1.4 mya (Bellomo 1994) (Gowlett 1984) but it is controversial. 
Acheulian Culture was clearly far more cognitively advanced than Oldowan culture, with great knapping dexterity, extreme mobility, systematic tracking skills and gestural languages being its main cognitive features. Marwick (Marwick 2003) presented good evidence of trade or resource-exchange routes of over a hundred kilometres existing after 1mya. Erectus would have been using simple but arbitrary path-marking signs, such as stone cairns and blazemarks on trees, to reduce the cognitive load on route-finding memory when travelling long distances. Marwick argues that a linguistic 'proto-language' that enabled this trading must have already emerged. Goen-Inbar thinks the level of stone-knapping technology at GBY also indicates linguistic communication. However, how does one 'tell' someone else how to ride a bicycle? I think all their evidence could also indicate the wide-spread use of gestural languages for trading communication, which later on became the template for speech.

I believe the evidence presented in chapter three and the material above can leave no doubts whatsoever that the Oldowan hominins were true hunters and gatherers. Only the prior evolution of a unique cognitive capacity or 'mechanism' for intentional mimetic transmission of new extractive foraging techniques, both horizontally and vertically, can explain how preOldowan hominins could culturally cope with the catastrophic changes in their local environments after 2.8 mya (Donald1991).

To summarize, I argue:

(1) That fundamental cognitive capacity was the HNF, triggered by social TWR and safe orienteering when foraging.

(2) It had evolved and become somewhat genetically/neurally entrenched over a couple of million years in the pre-Oldowan semi-aquatic socio-ecological niche.

(3) Environmental catastrophes after 2.8 Ma forced habitual application of TWR and the HNF to the extractive foraging domain; in particular, intentionally tracking down, killing, and butchering large mammals.

(4) This hugely successful cultural adaptation selectively triggered full entrenchment of the HNF and the neo-cortical expansion that led to the true hominins habilis and erectus by around 2 mya. 
(5) By then the co-evolutionary gene/culture/niche construction feed-back loop (Laland et al 2010) was fully in place, and led inexorably to all further changes in hominin evolution (see chronological chart following).

\section{CHRONOLOGICAL CHART OF HOMININ EVOLUTION}

\begin{tabular}{|c|c|c|c|c|}
\hline $\begin{array}{l}\text { Date and } \\
\text { Predominant } \\
\text { Feature }\end{array}$ & $\begin{array}{l}\text { Morphological } \\
\text { Attributes }\end{array}$ & $\begin{array}{l}\text { Archaeological } \\
\text { Evidence }\end{array}$ & $\begin{array}{l}\text { Ethnographic } \\
\text { Evidence }\end{array}$ & $\begin{array}{l}\text { Neurological/Cognitive } \\
\text { Attributes }\end{array}$ \\
\hline $\begin{array}{l}4.6 \text { to } 2.6 \mathrm{Ma} \\
\text { Full Terrestrial } \\
\text { Bipedalism in } \\
\text { Wetland Open } \\
\text { Environments } \\
\text { due to Massive } \\
\text { Volcanic Events } \\
\text { around } 4.5 \mathrm{Ma}\end{array}$ & $\begin{array}{l}\text { Free handedness } \\
\text { Precision Grip, } \\
\text { Accurate } \\
\text { Throwing, Long } \\
\text { Distance Carrying } \\
\text { Capacity, Some } \\
\text { Communal } \\
\text { Provision of } \\
\text { Young, Loss of } \\
\text { Laryngeal Air- } \\
\text { sacs, Sweating, } \\
\text { Hairlessness, due } \\
\text { to Semi-aquatic } \\
\text { Foraging }\end{array}$ & $\begin{array}{l}\text { Lacustrine Sites of } \\
\text { Earliest Fossilized } \\
\text { Skeletal Remains } \\
\text { and All Later Plio- } \\
\text { Pleistocene } \\
\text { Oldowan Stone } \\
\text { and Bone Caches; } \\
\text { Laetoli Fossilized } \\
\text { Footprints, } 3.4 \\
\text { mya Cut-marked } \\
\text { Bones, 1.95 site } \\
\text { with Bones of } \\
\text { Catfish, Turtles } \\
\text { and Crocodiles }\end{array}$ & $\begin{array}{l}\text { Nearly Universal } \\
\text { Exploitation of } \\
\text { Aquatic Fauna } \\
\text { and } \quad \text { Flora, } \\
\text { Historical and } \\
\text { modern Home } \\
\text { Bases Usually By } \\
\text { Resource-Rich } \\
\text { Water-ways }\end{array}$ & 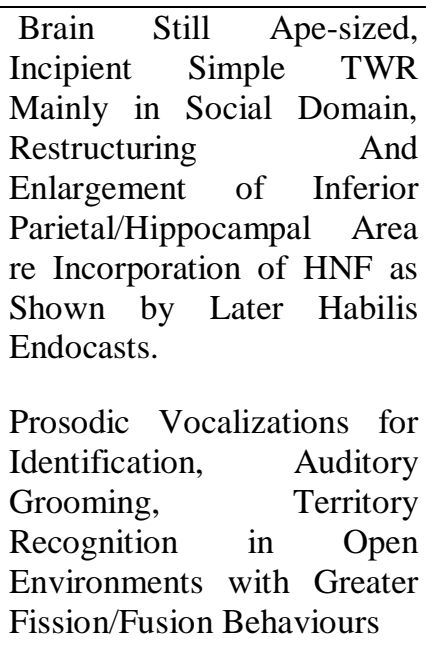 \\
\hline $\begin{array}{l}2.6 \text { to } 2 \mathrm{Ma} \\
\text { Oldowan Lithic } \\
\text { Culture }\end{array}$ & $\begin{array}{l}\text { Brain Doubled in } \\
\text { Size, Smaller Gut, } \\
\text { Enlarged Pre- } \\
\text { Frontal Cortex }\end{array}$ & $\begin{array}{l}\text { Large Carcass } \\
\text { Butchering Sites, } \\
\text { Long-Distance } \\
\text { Stone Resource } \\
\text { Acquisition, } \\
\text { Planned, Highly } \\
\text { Skilled Stone- } \\
\text { Knapping }\end{array}$ & $\begin{array}{l}\text { Highly Effective } \\
\text { Co-operative But } \\
\text { Technologically } \\
\text { Simple Hunting } \\
\text { Techniques for } \\
\text { Obtaining both } \\
\text { Large and Small } \\
\text { Animals due to } \\
\text { Loss of Wetland } \\
\text { Resources }\end{array}$ & $\begin{array}{l}\text { Encephalization Doubled in } \\
\text { Habilis and Erectus, Mainly } \\
\text { Due to Expanded Neo- } \\
\text { Cortex, HNF for } \\
\text { Anticipatory Cognition Fully } \\
\text { Entrenched and Modular, } \\
\text { Narrative Mimetic/Depictive } \\
\text { Communication, Beginning } \\
\text { of Systematic TWR Skill, } \\
\text { Refuge Bases }\end{array}$ \\
\hline $\begin{array}{l}2.0 \text { to } 1.6 \mathrm{Ma} \\
\text { True Hominins, } \\
\text { Habilis and } \\
\text { Erectus, Latter } \\
\text { Migrates } \\
\text { Throughout } \\
\text { Subtropical } \\
\text { OldWorld }\end{array}$ & $\begin{array}{l}\text { As Above, With } \\
\text { Erectus Reaching } \\
\text { Full Human Size, } \\
\text { Robust Endurance } \\
\text { Running Skeletal } \\
\text { Structure, } \\
\text { Altriciality, Post- } \\
\text { Menopausal } \\
\text { Grandmothers, } \\
\text { Hidden Oestrus }\end{array}$ & $\begin{array}{l}\text { Developed } \\
\text { Oldowan } \\
\text { throughout Old } \\
\text { World, Acheulian } \\
\text { Hand-axes in } \\
\text { Africa, Erectus } \\
\text { Very Wide- } \\
\text { Ranging, 1.5 Ma } \\
\text { Footprint, Turkana } \\
\text { Boy. }\end{array}$ & $\begin{array}{l}\text { As above, With } \\
\text { Addition of } \\
\text { Habitual } \\
\text { Confrontational } \\
\text { Scavenging as } \\
\text { well } \\
\text { Endurance as } \\
\text { Hunting begins } \\
\text { Around 1.7 mya } \\
\text { (Tapeworm) }\end{array}$ & $\begin{array}{l}\text { Systematic TWR for } \\
\text { Habitual Endurance Hunting } \\
\text { and Foraging Navigation in } \\
\text { Novel Environments, } \\
\text { Perhaps Opportunistic } \\
\text { Exploitation of Natural Fires, } \\
\text { Plaiting and Knotting } \\
\text { Technology, Chant/Ritual } \\
\text { Grooming, Neo-cortex } \\
\text { Continues to Expand }\end{array}$ \\
\hline
\end{tabular}




\begin{tabular}{|c|c|c|c|c|}
\hline $\begin{array}{l}1.6 \mathrm{Ma} \text { to } .8 \mathrm{Ma} \\
\text { Acheulian } \\
\text { Culture. } \\
\text { Fire by .9mya, } \\
\text { Entry into } \\
\text { Colder Old } \\
\text { World, Larger- } \\
\text { Brained } \\
\text { heidelbergensis } \\
\text { in Europe, } \\
\text { England by .9 } \\
\text { mya }\end{array}$ & $\begin{array}{l}\text { Thick-skulled, } \\
\text { Highly Robust, } \\
\text { Full HG Life-way } \\
\text { indicated by } \\
\text { Home-Based Fire } \\
\text { Control, } \\
\text { Knotting/Plaiting } \\
\text { Technologies? }\end{array}$ & $\begin{array}{l}\text { Gesher Site } .9 \text { mya } \\
\text { Proves Fire use, } \\
\text { Nut-meat } \\
\text { Grinding, } \\
\text { Systematic } \\
\text { Exploitation of } \\
\text { Fallow Deer and } \\
\text { Gazelle, Turtle } \\
\text { and Ostrich Shell }\end{array}$ & $\begin{array}{l}\text { As above, Plus } \\
\text { Preservation } \\
\text { Techniques } \\
\text { (Drying of Meat), } \\
\text { Processing of } \\
\text { Tubers, } \\
\text { Exploitation of } \\
\text { Cold } \\
\text { Environments }\end{array}$ & $\begin{array}{l}\text { All of the Above, Big-Game } \\
\text { Hunting for Status and } \\
\text { Extra-Marital Copulations, } \\
\text { Marriage and Punishment } \\
\text { Rituals, Home Base Sociality } \\
\text { Gestural Languages for } \\
\text { Trade, Reciprocal } \\
\text { Relationships with Strangers } \\
\text { Large fission/fusion tribal } \\
\text { gatherings? }\end{array}$ \\
\hline 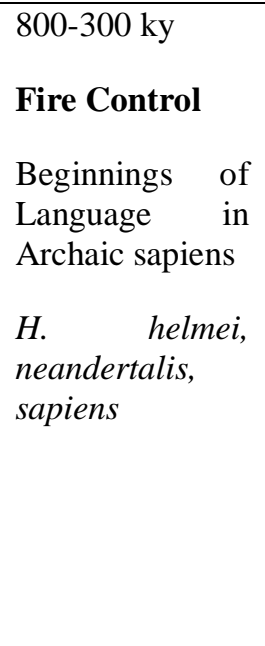 & $\begin{array}{lr}\text { Final Brain } & \text { Surge } \\
\text { Occurs, but Archaic } \\
\text { Sapiens less thick } \\
\text { skulled, } & \text { Less } \\
\text { Robust than } & \text { helmei/neandertalis, } \\
\text { Beginnings } & \text { of } \\
\text { Aesthetically } & \\
\text { Pleasing Hand- } & \text { Axes, } \\
\text { technology, Snares, } \\
\text { Finest Acheulian in } \\
\text { Europe, Javelin-like } \\
\text { Spears }\end{array}$ & $\begin{array}{l}\text { Enlarged and } \\
\text { Thinner Skulls, } \\
\text { Bone Residues } \\
\text { Show Continuous } \\
\text { Big-Game } \\
\text { Procurement, } \\
\text { Grinding stones } \\
\text { Ubiquitous=More } \\
\text { Intensive } \\
\text { Foraging in Cold } \\
\text { Environments, } \\
\text { Hyoid Bone } \\
\text { Shows Greater } \\
\text { Phonological } \\
\text { Complexity of } \\
\text { Vocalizations }\end{array}$ & $\begin{array}{l}\text { As above, but } \\
\text { more intensive, } \\
\text { Shell-fish } \\
\text { Middens Near } \\
\text { Permanent } \\
\text { Refuge Caves, } \\
\text { More Role } \\
\text { Specialization, } \\
\text { Rituals, Division } \\
\text { of Labour } \\
\text { between Hunters } \\
\text { and Tool-makers, } \\
\text { etc. as per } \\
\text { Modern HGs. }\end{array}$ & \begin{tabular}{lr} 
As above, but more \\
intensive, \\
systematic/speculative TWR, \\
Beginnings of Linguistic \\
Communication & from \\
Phonological & Prosody \\
(Chanting) and Mimicking \\
of Animals/Natural Sounds. \\
\multicolumn{2}{l}{ Vocalizations mapped on to } \\
Narrative Mimetic/depiction \\
$\begin{array}{l}\text { Modes and Gestural } \\
\text { Languages }\end{array}$
\end{tabular} \\
\hline $\begin{array}{l}300 \text { ky to } 20 \mathrm{ky} \\
\text { Full } \\
\text { Emergence of } \\
\text { Language } \\
\text { True Sapiens, } \\
\text { Neandertals } \\
\text { Begin to Die } \\
\text { Out, Extinction } \\
\text { of Erectus in } \\
\text { Eastern Old } \\
\text { World }\end{array}$ & $\begin{array}{l}\text { Fully Modern } \\
\text { Sapiens at } 195 \mathrm{ky}, \\
\text { Neandertalis dies } \\
\text { Out 25ky }\end{array}$ & $\begin{array}{l}\text { Ochre Widely } \\
\text { Used, Behavioural } \\
\text { Modernity } \\
=\text { Symbolic } \\
\text { artefacts Hafted } \\
\text { Tools, Fine-blade } \\
\text { Technology from } \\
100 \text { ky on }\end{array}$ & $\begin{array}{l}\text { Full HGs as per } \\
\text { Historical and } \\
\text { Modern } \\
\text { Examples, } \\
\text { Occupation of } \\
\text { All } \\
\text { Environments, } \\
\text { Intensification } \\
\text { of all of the } \\
\text { Above }\end{array}$ & $\begin{array}{l}\text { Behavioural Modernity, } \\
\text { Beginning of Second } \\
\text { Diaspora Out of Africa at } \\
100 \mathrm{ky} \text {, Cave Art at } 45 \mathrm{ky} \text {, } \\
\text { Domestication of dogs } \\
\text { 30ky(?) Sedentary Living } \\
\text { and Beginnings of Pseudo- } \\
\text { Horticulure in Middle East } \\
20 \mathrm{ky} \text {. }\end{array}$ \\
\hline
\end{tabular}

\subsection{Discussion of Chronology}

My chronology differs largely from (Foley and Gamble 2009) in only one major particular: I argue that hominins were (a) hunters of small aquatic and terrestrial animals, very infrequent opportunistic carvers of large carcasses until 2.6 mya, (b) habitual hunters of medium to large animals, probably using the bog-miring technique from then until around 1.8 mya; and (c) 
persistence or endurance hunters combined with confrontational/obligate scavenging after 1.8 mya. Intentional acquisition of large carcasses at 2.5 mya was due to environmental forcing from loss of preferred wetland resources.

I also add these TWR stages: (1) simple for social/foraging orienteering before 2.6 mya; (2) simple to systematic social+hunting 2.6 to 1.6 mya; and (3) systematic to speculative fullblown TWR thereafter. There was co-evolving neural restructuring and neo-cortical expansion for increasing TWR skills and the applications of TWR information by the HNF in all facets of the expanding, eusocial carnivore niche of the hominin lineage.

I also argue for a gestural Acheulian Culture, which is probably not so important a difference. Where we agree most strongly is in the timing of the second brain-surge from .8 Ma onwards. I argue that this was mainly due to three selective factors:

1) There was another major adaptive cliff that occurred at $.8 \mathrm{Ma}$, the Matayuma-Brunhes Polarity Reversal, which inaugurated the glacial, extremely tempestuous climactic character of the mid to late Pleistocene

2) This triggered every-day systematic control of fire, which made possible more daytime hours of vocal chanting, dancing and ritual due to safety from predators, plus preservation and exploitation of a much larger range of resources, in suddenly much colder environments. This innovation opened the door to supplying the necessary high-quality diet for another surge in encephalization

3) Linguistic communication emerged as a way of communicating when not in the light of the fire. It rode on the back of a) neural expansion in the neo-cortex; b) the already well-developed phonological complexity of prosodic vocalizations (singing and chanting) and of animal mimicry when hunting; c) an already well-developed gestural language base used for trade with other tribes and silent communication when hunting.

There are many examples of sign-languages that could be understood continent-wide between historical HG tribes with discreet linguistic modes of communication, throughout North America, Australia, The Middle East, India, and so on.

To reiterate: all spontaneous sign-languages (like many linguistic languages) have an SOV (Subject-Object-Verb) grammar. What is most important in any communicative exchange is who the main characters in the narrative being described are, and then comes the action. We 
are using SVO here, but all six possible combinations occur in the world's languages. However, people of all languages revert to SOV when asked to communicate in mime or gesture. I would argue that this is due to the core structure of the HNF. However, language would have required good phonological complexity as well as cumulative mimetic/depictive and gestural cultural support for its invention.

\subsection{The Lacustrine Vocalizing Ape}

This section is not crucial to my thesis, but it completes my narrative of the evolution of cognitive capacities for linguistic communication. The other hominin social marker beside conspecific track-ways would have been vocal. I speculate that living and foraging in open wetland environments selected for highly vocal social communication behaviours. I argue for convergent evolution here, analogously to the howling of dogs, singing of killer whales, and the continual highly complex vocalizing of Gelada baboons. They live in troupes of up to 250 individuals in very open habitats, and the complexity and range of their vocalizing is remarkably similar to the audile contours of human language (Rendall, Kollias et al. 2005).

Such vocalizing or 'singing' is a form of social grooming as well as a navigational/territorial location marker in matrilinear Gelada societies. They recognize as each other as individuals by the sound of their voices. Since they sleep or roost in small kin-groups in rock overhangs on sheer cliffs at night, they begin and end each day with dawn and evening choruses, somewhat like birds.

I am thinking of mimetic ululating Oldowans here, and then Acheulian gestural cultures that began dancing and chanting as a form of social grooming, especially in the evening, around fires after about .9 mya. The first words were probably onomatopoeic (a word that sounds like what it refers to) due to already well-developed vocal mimicry of other animals and natural sounds, something we hominins became extremely good at. I repeat: one of our favourite hunting techniques is to lure animals to within killing range by mimicking their mating calls.

I bet pre-hominin reciprocal and recognizable vocalizing, especially between mothers and infant $^{74}$, was a precursor to mimicry of other animal's calls, extremely keen voice recognition and the culturally signifying dialects within languages. This vocalizing later (much later) made the dialogic exchanges of linguistic communication possible, due to its

\footnotetext{
${ }^{74}$ This idea for language evolution is known as the "Motherese Hypothesis".
} 
probable Gelada-like phonological complexity. Hominin infants could not cling to the hair of mothers like those of other apes, and open flood-plains and swampy environments habitats are characterized by vast expanses of tall reeds, sedges and grasses that are easy to get lost in. HG women and children talk or chant continuously when gathering together in order to deter predators (Botha and Knight 2009, Silberbauer 1981)

In short, hominin prosodic vocalizing would have been an important pre-adaptation for language invention further down the evolutionary trail, acquired in conjunction with other important traits such as simple TWR, through wetland foraging habits. Apart from a need for this pre-adaptation for the phonetic complexity of speech, I fully support Merlin Donald's mimetic and Michael Corballis's gesture-first 'evolution of language' theories.

\subsection{The Socio-Ecological Effects of Linguistic Communication}

There was positive feedback between linguistic communication, the surge in encephalization mentioned above, and culture. Speech demands much less physical energy than mime or gesture (Armstrong et al 1995). Language also allows for a lot of fine-grained cryptic encoding, even within the intimate social structure of an HG band (see Botha and Knight 2009, Silberbauer 1981 for ethnographic examples). So obviously its inception increased social and technological complexity. Donald (in Corballis and Lea 1991) suggests postmimetic and depictive hominin culture should be called mythic culture. I agree, if we can place a predominantly gestural Acheulian culture in between (we will get back to mythic culture later in this section)

One of the puzzling features of this period of hominin evolution is the numerous sites where many of these hand-axes were made and never used; also they became more beautiful and aesthetically pleasing as time went on. Wynn (Wynn 2002) suggests that these axes were the first symbolic art, and may have been honest sexual signals of dexterity and intelligence. I would agree, but would add that axe-knapping may have become more skilled as hands were freed from communication; hominins could knap and communicate simultaneously.

Indeed, speech may have promoted technological innovation more generally, by freeing the hands for experiment. One could talk and still need 'something to do with the hands'. Armstrong (et al 1995) and Corballis (Corballis 2010) have also presented this idea. There is a natural progression to simple games for auto-rehearsal here as well. Cats-cradle (for developing weaving skills) and drawing track-ways in sand, dust or snow (for TWR) can be placed in this cognitive basket. Group games or 'sport' incorporate auto-rehearsal of skills as 
well. However, we must not forget the possible importance of what is being talked about during these activities. I refer to Donald's mythic culture.

Mythic culture is constructed around narratives that are endlessly reiterated using combinations of mime, gesture, symbolic depictions, and language. These cultural narratives attain emotional or social-grooming power through ritualistic 'dressing-up', dancing and chanting, and their story-lines are often expressed through songs or poetry. They provide tribal identity: they express group-narratives of 'who we are and where we come from'.

Cultural myths narratively present normative social roles for individuals to use as 'maps' for a life time of navigation through the social domain. Maturing individuals' self-narratives of 'how do I get to fit in to the group' are thereby socially manipulated ${ }^{75}$ for the good of the tribe. Consider marriage contracts and punishment/reward social institutions such as group ridicule here. In HG tribes it is the older women who usually apply the social-ridicule technique when dealing with younger band-members to 'bring them into line'. They do this with exaggerated mimicry of the miscreant and loud, humorous story-telling so all band members can hear and join in the laughter (Botha and Knight 2009 for excellent description).

Smaller mythic tales such as hunting stories and family genealogies are endlessly re-told around HG campfires (and modern kitchen tables) at night. There are also the narratives of gossip. These smaller narratives, communicated via speech alone or with a few gestures, are a subtle form of pedagogy, since they contain useful information for children and juveniles about kin-relationships, folk psychology and local ecologies.

The communication of human culture is therefore narratively constructed. At bottom the template for all this narrativity is the primal autonoetic, autobiographical self-narrative. The up-shot is that in modern, complex societies the individual autonoetic self-narrative at the core of the HNF is in constant narrative discourse with itself and with the 'autonoetic selfnarratives' of various social groups. We are the autonoetic or self-knowing narrative species.

The impact of language on symbolic signs of self, tribal identity, and therefore on hominin autonoetic self-narratives would have to be the subject of another paper. I will finish this section on linguistic forms of narrative communication with (a) a few notes on how my 'triggering track-ways theory' solves certain long-standing problems in the 'evolution of

\footnotetext{
${ }^{75}$ Like the self-narrative, these cultural 'autobiographical' narratives must be adjusted over time. But the process can be very 'socially messy', to say the least.
} 
language' debate; and (b) a brief comment on why I think the 'episodic faculty' should be called the hominin narrative faculty.

(Hauser, Chomsky et al. 2002) argued that what was truly unique about language was its recursive nature. They stated the cognitive capacity for understanding this recursion (syntax or grammar) must have evolved before language could emerge, in the either the social, numerical, or navigation domains. My TWR theory explains how and why the most important feature of language could only have evolved in the social and navigation domains. Deacon (1997) stated there was no natural sign-system in the real world on to which the arbitrary sign-systems of language could be mapped. He ended up having to posit a very tangled view of the evolution of symbols. This paper has revealed that he was wrong.

There has always been a discrete infinity of stories to be read in the naturally recursive or combinatory sign-system of foot-prints and track-ways, just as there is in the recursively embedded conventional or symbolic words and sentences of language. I argue the cognitive capacity for language acquisition, both phylogenetically and ontogenetically, was built on the ancient cognitive capacity for TWR.

Some arbitrary signs were probably in use very early, to decrease the cognitive load on narrative, navigational memory when travelling between important sites. I have in mind such structures as stone cairns, blaze-marks on trees, and the like. Early use of such simple signs is very probable in open featureless wetland environments. Here too there is a cognitive connection between reading track-ways sign systems and 'reading' or understanding the arbitrary, symbolic sign systems of language. This development culminated in orthography and all the other depictive signs used in modern culture. So the word-signs you are reading right now are 'thought-prints', and the 'track-way' they are making is my thesis or evolutionary narrative.

"True beliefs are not pictures, mirrors or maps. But a true belief in the naturalistic view we shall discuss, has the same relation to the world that an accurate map has"

(Peter Godfrey-Smith 1996)

My point here is, so does a true story, and I believe the triggering track-ways theory tells a true story. Like all true stories, it appeals to common sense: it is not another 'just-so' story. It is also theoretically orthodox in all its facets. I will finish my thesis with a précis of my evolutionary narrative. 


\subsection{Conclusion}

Four and a half million years ago a massive volcanic event destroyed forests throughout the East Afican Rift. Consequently some of our facultatively bipedal, semi-arboreal Ardipithecan ancestors became non-arboreal, obligate bipedal apes, and got locked into specializing in their semi-aquatic, wetland socio-ecological niche. Foraging in and under water led to other morphological adaptations, such as copious sweating, hairlessness, breathe control, loss of laryngeal air-sacs, highly sensitive finger pads, a strong flexible wrist and a precision grip.

These earliest hominins also, of course, possessed universal great-ape cognitive capacities for here-and-now imitation and intentionality-reading. Compared to other higher mammals, they were good here-and-now social learners. These pre-adaptive physical and mental traits were all necessary for the evolution of hominin cognition, culture and communication, but they were not sufficient.

Wetlands are boggy featureless environments surrounding lakes and rivers. They are covered in tall sedges and reeds, and water-levels fluctuate constantly. The ubiquitous sandy and muddy substrates in wetlands lend themselves to accurate recording of footprints and trackways. These ever-present natural signs induced some populations of wetland-foraging hominins to enter a unique cognitive/behavioural niche. In fact, this niche probably had to be entered by a bipedal, visually oriented, non-olfactory ape that habitually foraged in such environments.

Consequently the earliest hominins began imitatively stepping in or very near the footprints of leading conspecifics, on a routine basis. This behaviour was immediately selected for because it increased fitness when foraging. Soon they were also exploiting their own old track-ways and those of absentee band-members for safety and orienteering, in the same imitative/emulative manner. They also began to use conspecific track-ways as social markers because they were individually recognizable.

Unlike scent trails track-ways are immediately directional, durable, and combinatory. They have a narrative structure, and therefore reveal what their authors were doing and experiencing when they were made. Hominins walking in the old prints of absentee bandmembers and their own old prints were physically re-enacting the stories being told by the track-ways being followed. Through this continuous embodied cognition early hominin mind/brains slowly began to develop the elsewhere-and-when cognitive capacity for imagining 'being in the minds-and-bodies' of absentee band members and their past selves. 
Track-ways are immediately and inexorably directional because every footprint points to where its author was heading at the time it was made. They therefore kindled an objective awareness of the self and others as intentional agents, acting out scenarios in the past in a narrative, goal-directed manner. When leaving and returning to the social domain early hominins (I do not include australopithecines in the hominin lineage) very often exploited their own recognizable old track-ways. Due to this self-mirroring effect of their own trackways the faculty for an autonoetic narrative memory and Dennett's second-order intentionality began to evolve.

Since the autonoetic self-narrative was constructed of memories of the imagined self experiencing the past, it also allowed imagining the intentional self having experiences in the future. This provided anticipatory cognition, or Suddendorf's mental time-travel. It also allowed the attribution of intentional mental states to both present and non-present others. The evolution of the unique hominin narrative faculty for elsewhere-and-when social competences had been triggered. Hominins were no longer restricted to the here-and-now imitation/emulation and intentionality-reading of adult chimps and human toddlers under the age of three.

The outward expression of the hominin narrative faculty was mimetic pretend play, autorehearsal, and communication, via synchronous turn-and-turnabout imitations of the imagined actions of absentee entities (including the self) during past and future events. Capacities for social learning increased dramatically. Internally hominins began to cognitively 'live in time' due to their evolving neural capacity for mental representations of an autonoetic, autobiographical self-narrative. Social complexity increased well beyond that of any other great ape societies, and a cognitive arms race ensued.

The hominin narrative faculty and the concomitant capacity for intentional (rather than opportunistic) reading of conspecific track-ways began to co-evolve, in a selective feed-back loop. Two million years later, the hominin narrative faculty and its spin-off elsewhere-andwhen social competences were entrenched enough to enable the abrupt emergence of mimetic Oldowan Culture. This explosion of co-operative culture enabled hominins to survive drastic environmental changes in the East African Rift throughout the Late Pliocene and Early Pleistocene.

These environmental catastrophes were caused by a north-south polar reversal at 2.8 mya. Oldowan hominins had to become intentional trackers and co-operative hunters of large 
mammals, due to destruction of their staple diet of wetland resources. These narratively planned and mimetically communicated co-operative foraging techniques were a huge fuel for success (Godfrey 1996). Powerful selection forces therefore arose for rapid encephalization and earlier birthing/extended juvenility, to increase the plastic neural capacity to learn those techniques when young.

By around 2 mya the true hominins habilis and erectus had evolved, with twice the encephalization of other great ape species (including australopithecines). Mimetic Oldowan HG culture cumulatively developed into gestural Acheulian Culture by 1.6 mya. By then the hominin narrative faculty was fully entrenched. Erectus hominins had become eusocial breeders, systematic intentional trackers, wide-ranging endurance hunters and confrontational scavengers. They had colonized all warmer parts of the Old World. Their morphology and developmental life history was almost human, for extended childhood dependency and compensatory alloparenting by post-menopausal grandmothers had fully evolved. Further encephalization was occurring, but very incrementally.

At .78 mya another polar reversal occurred that caused massive glaciations in the Northern Hemisphere throughout the rest of the Pleistocene. Erectus responded by taking full control of fire. Fire allowed innovative extraction of high-quality nutrients from a wider range of environmental resources, more time for noisy socializing, and therefore amped up socioecological complexity. Consequently another surge in encephalization began. Late Acheulian hominins became fully fledged systematic/speculative trackers and started to invent language. By 400 to 300 ky archaic sapiens and neandertalis had evolved.

Their brains had become three times as large as that of a chimpanzee, partly due to a cognitive arms-race because language was being invented. The result of such energy-efficient symbolic communication was complex mythic culture and the emergence of the technological and symbolic cultural innovations of behavioural modernity by $100 \mathrm{ky}$.

When language emerged we fully became, as Deacon (1997) has so aptly said, the symbolic species. What my triggering track-ways theory has added to human evolutionary theory is that we had become a somewhat elsewhere-and-when, narrative-minded species long before the invention of Oldowan Culture, never mind language, because our earliest ancestors had entered the unique cognitive/behavioural niche of exploiting their own track-ways some two million years before Oldowan Culture. 
I argue that if our ancestral ape lineage had not entered this unique cognitive niche at that early point in its evolutionary trajectory, cumulative hominin cultures and intentional communication would never have emerged, and H. sapiens could not have evolved. This new evolutionary narrative is based on these now orthodox evolutionary theories: niche construction, environmental variability, social complexity, and selective feedback spiralling between biological and cultural evolution in four dimensions: genetic, epigenetic, cultural, and communicational. If it is a true story then the 'triggering track-ways theory' provides the missing piece to the evolutionary puzzle of why we are the way we are: the autonoetic narrative-minded symbolic species.

******************************

Acknowledgements: My thanks to Kim Sterelny and Ben Jeffares for their patience, and so gently goading me towards an understanding of what I would never have otherwise understood about writing a thesis. 


\section{Bibliography}

Addis, D. R., L. Pan, et al. (2009). "Constructive episodic simulation of the future and the past: Distinct subsystems of a core brain network mediate imagining and remembering." Neuropsychologia 47(11): 2222-2238.

Addis, D. R., A. T. Wong, et al. (2007). "Remembering the past and imagining the future: Common and distinct neural substrates during event construction and elaboration." Neuropsychologia 45(7): 1363-1377.

Alemseged, Z., F. Spoor, et al. (2006). "A juvenile early hominin skeleton from Dikika, Ethiopia." Nature 443(7109): 296-301.

Armstrong, D. F., W. C. Stokoe, et al. (1995). Gesture and the nature of language. Cambridge ; New York, NY, USA, Cambridge University Press.

Atance, C. M. and D. K. O'Neill (2005). "The emergence of episodic future thinking in humans." Learning and Motivation 36(2): 126-144.

Baddeley, A. (2000). "The episodic buffer: a new component of working memory?" Trends in Cognitive Sciences 4(11): 417-423.

Baillargeon, R., R. M. Scott, et al. (2010). "False-belief understanding in infants." Trends in Cognitive Sciences 14(3): 110-118.

Bates, L. A. and R. W. Byrne "Imitation: what animal imitation tells us about animal cognition." Wiley Interdisciplinary Reviews: Cognitive Science 1(5): 685-695.

Behrensmeyer, A. K., N. E. Todd, et al. (1997). "Late Pliocene Faunal Turnover in the Turkana Basin, Kenya and Ethiopia." Science 278(5343): 15891594.

Bellomo, R. V. (1994). "Methods of determining early hominid behavioral activities associated with the controlled use of fire at FxJj 20 Main, Koobi Fora, Kenva." Journal of Human Evolution 27(1-3): 173-195. 
Bennett, M. R., J. W. K. Harris, et al. (2009). "Early Hominin Foot Morphology Based on 1.5-Million-Year-Old Footprints from Ileret, Kenya." Science 323(5918): 1197-1201.

Botha, R. P. and C. Knight (2009). The prehistory of language. Oxford ; New York, Oxford University Press.

Braun, D. R., J. W. K. Harris, et al. (2010). "Early hominin diet included diverse terrestrial and aquatic animals $1.95 \mathrm{Ma}$ in East Turkana, Kenya." Proceedings of the National Academy of Sciences of the United States of America 107(22): 10002-10007.

Broadhurst, C. L., S. C. Cunnane, et al. (1998). "Rift Valley lake fish and shellfish provided brain-specific nutrition for early Homo." British Journal of Nutrition 79(01): 3-21.

Buckner, R. L. (2007). "Prospection and the brain." Behavioral and Brain Sciences 30(03): 318-319.

Buckner, R. L. and D. C. Carroll (2007). "Self-projection and the brain." Trends in Cognitive Sciences 11(2): 49-57.

Call, J., B. Hare, et al. (2004). "'Unwilling' versus 'unable': chimpanzees' understanding of human intentional action." Developmental Science 7(4): 488-498.

Call, J. and M. Tomasello (2008). "Does the chimpanzee have a theory of mind? 30 years later." Trends in Cognitive Sciences 12(5): 187-192.

Calvin, W. H. and D. Bickerton (2000). Lingua ex machina : reconciling Darwin and Chomsky with the human brain. Cambridge, Mass., MIT Press.

Carpenter, M., N. Akhtar, et al. (1998). "Fourteen through 18-month-old infants differentially imitate intentional and accidental actions." Infant Behavior \& Development 21(2): 315-330.

Corballis, M. C. (1992). "On the evolution of language and generativity." Cognition 44(3): 197-226.

Corballis, M. C. (2002). From hand to mouth : the origins of language. Princeton, Princeton University Press.

Corballis, M. C. (2003). "From mouth to hand: Gesture, speech, and the evolution of right-handedness." Behavioral and Brain Sciences 26(02): 199-208.

Corballis, M. C. (2010). "The gestural origins of language." Wiley Interdisciplinary Reviews: Cognitive Science 1(1): 2-7.

Corballis, M. C. and S. E. G. Lea (1999). The descent of mind : psychological perspectives on hominid evolution. Oxford ; New York, Oxford University Press.

Cunnane, S. C. and K. M. Stewart (2010). Human brain evolution : the influence of freshwater and marine food resources. Hoboken, N.J., WileyBlackwell. 
Deacon, T. (1997). The Symbolic Species: The co-evolution of language and the human brain, Penguin.

Dennett, D. C. (1987). The intentional stance. Cambridge, Mass., MIT Press.

Dennett, D. C. (1991). Consciousness explained. Boston, Little, Brown and Co.

Diez-Martín, F., P. Sánchez, et al. (2009). "Were Olduvai Hominins making butchering tools or battering tools? Analysis of a recently excavated lithic assemblage from BK (Bed II, Olduvai Gorge, Tanzania)." Journal of Anthropological Archaeology 28(3): 274-289.

Dominguez-Rodrigo, M., H. T. Bunn, et al. (2010). "Paleoecology and hominin behavior during Bed I at Olduvai Gorge (Tanzania)." Quaternary Research 74(3): 301-303.

Domínguez-Rodrigo, M., A. Mabulla, et al. (2009). "Unraveling hominin behavior at another anthropogenic site from Olduvai Gorge (Tanzania): new archaeological and taphonomic research at BK, Upper Bed II." Journal of Human Evolution 57(3): 260-283.

Domínguez-Rodrigo, M. and B. Martínez-Navarro "Taphonomic analysis of the early Pleistocene (2.4 Ma) faunal assemblage from A.L. 894 (Hadar, Ethiopia)." Journal of Human Evolution In Press, Corrected Proof.

Donald, M. (1991). Origins of the modern mind : three stages in the evolution of culture and cognition. Cambridge, Mass., Harvard University Press.

Ennion, E. A. R. and N. Tinbergen (1967). Tracks. Oxford,, Clarendon P.

Foley, R. and C. Gamble (2009). "The ecology of social transitions in human evolution." Philosophical Transactions of the Royal Society B-Biological Sciences 364(1533): 3267-3279.

Gilmore, H. W. (1953). "Hunting Habits of the Early Nevada Paiutes*." American Anthropologist 55(1): 148-153.

Godfrey-Smith, P. (1996). Complexity and the function of mind in nature. Cambridge ; New York, Cambridge University Press.

Goren-Inbar, N., C. S. Feibel, et al. (2000). "Pleistocene Milestones on the Outof-Africa Corridor at Gesher Benot Ya'aqov, Israel." Science 289(5481): 944-947.

Gowlett, J. A. J. (1984). "Mental abilities of early man." Higher Education Quarterly 38(3): 199-220.

Gurven, M., K. Hill, et al. (2002). "From forest to reservation: Transitions in food-sharing behavior among the Ache of Paraguay." Journal of Anthropological Research 58(1): 93-120.

Harris, M. D. L. a. J. M., Ed. (1987). Laetoli: A Pliocene Site in Northern Tanzania. Oxford Science Publications. New York, OUP.

Hauser, M. D., N. Chomsky, et al. (2002). "The Faculty of Language: What Is It, Who Has It, and How Did It Evolve?" Science 298(5598): 1569-1579.

Heinzelin, J. d., J. D. Clark, et al. (1999). "Environment and Behavior of 2.5Million-Year-Old Bouri Hominids." Science 284(5414): 625-629. 
Hernández Fernández, M. and E. S. Vrba (2006). "Plio-Pleistocene climatic change in the Turkana Basin (East Africa): Evidence from large mammal faunas." Journal of Human Evolution 50(6): 595-626.

Hewes, G. W. (1994). Evolution of human semiosis and the reading of animal tracks. Origins of Semiosis: Sign Evolution in Nature and Culture. W. Noth. Berlin; New York, Mouton de Gruyer: 139-150.

Hill, K., H. Kaplan, et al. (1987). "Foraging Decisions among Ache HunterGatherers - New Data and Implications for Optimal Foraging Models." Ethology and Sociobiology 8(1): 1-36.

Holloway, R. L., D. C. Broadfield, et al. (2003). "The lunate sulcus and early hominid brain evolution: Toward the end of a controversy." American Journal of Physical Anthropology: 117-117.

Horner, V. and A. Whiten (2005). "Causal knowledge and imitation/emulation switching in chimpanzees (Pan trogiodytes) and children (Homo sapiens)." Animal Cognition 8(3): 164-181.

Hrdy, S. B. (2009). Mothers and others : the evolutionary origins of mutual understanding. Cambridge, Mass., Belknap Press of Harvard University Press.

Jablonka, E. a. L., Marion J. (2006). "Precis of: Evolution in Four Dimensions." To be published in Behavioural and Brain Sciences.

Kaplan, H., M. Gurven, et al. (2010). "Learning, menopause, and the human adaptive complex." Reproductive Aging 1204: 30-42.

Kaplan, H. and K. Hill (1985). "Food Sharing among Ache Foragers - Tests of Explanatory Hypotheses." Current Anthropology 26(2): 223-246.

Kaplan, H. and K. Hill (1985). "Hunting Ability and Reproductive Success among Male Ache Foragers - Preliminary-Results." Current Anthropology 26(1): 131-133.

Kaplan, H., K. Hill, et al. (1984). "Food Sharing among Ache Hunter-Gatherers of Eastern Paraguay." Current Anthropology 25(1): 113-115.

Lancaster, J. B., H. S. Kaplan, et al. (2000). "The evolution of life history, intelligence and diet among chimpanzees and human foragers." Perspectives in Ethology, Vol 13 13: 47-72

318.

Lee, R. B., I. DeVore, et al. (1969). Man the hunter. Chicago,, Aldine Pub. Co.

Leonard, J. A., R. K. Wayne, et al. (2002). "Ancient DNA Evidence for Old World Origin of New World Dogs." Science 298(5598): 1613-1616.

Liebenberg, L. (1990). The art of tracking : the origin of science. Claremont, South Africa, D. Philip.

Liebenberg, L. (2008). "The relevance of persistence hunting to human evolution." Journal of Human Evolution 55(6): 1156-1159.

Marwick, B. (2003). "Pleistocene Exchange Networks as Evidence for the Evolution of Language." Cambridge Archaeological Journal 13(01): 6781. 
McCoy, F. and G. Heiken (2000). Volcanic hazards and disasters in human antiquity. Boulder, Colo., Geological Society of America.

McNeil, L. D. (1996). "Homo inventans: The evolution of narrativity." Language \& Communication 16(4): 331-360.

McPherron, S. P., Z. Alemseged, et al. (2010). "Evidence for stone-tool-assisted consumption of animal tissues before 3.39 million years ago at Dikika, Ethiopia." Nature 466(7308): 857-860.

Meltzoff, A. N. and J. Decety (2003). "What imitation tells us about social cognition: a rapprochement between developmental psychology and cognitive neuroscience." Philosophical Transactions of the Royal Society of London. Series B: Biological Sciences 358(1431): 491-500.

Morrison, R. G. B. (1981). A field guide to the tracks \& traces of Australian animals. Adelaide, Rigby.

Osvath, M. and P. Gärdenfors (2007). "What are the evolutionary causes of mental time travel?" Behavioral and Brain Sciences 30(03): 329-330.

Pickering, T. R. and H. T. Bunn (2007). "The endurance running hypothesis and hunting and scavenging in savanna-woodlands." Journal of Human Evolution 53(4): 434-438.

Plummer, T. (2004). "Flaked stones and old bones: Biological and cultural evolution at the dawn of technology." American Journal of Physical Anthropology 125(S39): 118-164.

Pobiner, B. L., M. J. Rogers, et al. (2008). "New evidence for hominin carcass processing strategies at 1.5 Ma, Koobi Fora, Kenya." Journal of Human Evolution 55(1): 103-130.

Potts, R. (1998). "Environmental hypotheses of hominin evolution." American Journal of Physical Anthropology 107(S27): 93-136.

Pruetz, J. D. and P. Bertolani (2007). "Savanna Chimpanzees, Pan troglodytes verus, Hunt with Tools." Current Biology 17(5): 412-417.

Quade, J., N. Levin, et al. (2004). "Paleoenvironments of the earliest stone toolmakers, Gona, Ethiopia." Geological Society of America Bulletin 116(11-12): 1529-1544.

Rakoczy, H. (2006). "Pretend play and the development of collective intentionality." Cognitive Systems Research 7(2-3): 113-127.

Rendall, D., S. Kollias, et al. (2005). "Pitch (F[sub 0]) and formant profiles of human vowels and vowel-like baboon grunts: The role of vocalizer body size and voice-acoustic allometry." The Journal of the Acoustical Society of America 117(2): 944-955.

Rose, L. and F. Marshall (1996). "Meat Eating, Hominid Sociality, and Home Bases Revisited." Current Anthropology 37(2): 307-338.

Schacter, D. L. and D. R. Addis (2007). "On the constructive episodic simulation of past and future events." Behavioral and Brain Sciences 30(03): 331-332. 
Schacter, D. L. and D. R. Addis (2009). "On the nature of medial temporal lobe contributions to the constructive simulation of future events." Phil Trans R Soc B 364(1521): 1245-1253.

Schacter, D. L., D. R. Addis, et al. (2007). "Remembering the past to imagine the future: the prospective brain." Nat Rev Neurosci 8(9): 657-661.

Schaller, G. B. and G. R. Lowther (1969). "Relevance of Carnivore Behavior to Study of Early Hominids." Southwestern Journal of Anthropology 25(4): 307-341.

Semaw, S. (2000). "The World's Oldest Stone Artefacts from Gona, Ethiopia: Their Implications for Understanding Stone Technology and Patterns of Human Evolution Between 2.6-1.5 Million Years Ago." Journal of Archaeological Science 27(12): 1197-1214.

Shepard, P. (1996). The others : how animals made us human. Washington, D.C., Island Press.

Shipman, P. (2009). "Cooking debate goes off the boil." Nature 459(7250): 1059-1060.

Shipman, P., W. Bosler, et al. (1981). "Butchering of Giant Geladas at an Acheulian Site [and Comments and Reply]." Current Anthropology 22(3): 257-268.

Silberbauer, G. B. (1981). Hunter and habitat in the central Kalahari Desert. Cambridge [Eng.] ; New York, Cambridge University Press.

Spreng, R. N. and C. L. Grady (2010). "Patterns of Brain Activity Supporting Autobiographical Memory, Prospection, and Theory of Mind, and Their Relationship to the Default Mode Network." Journal of Cognitive Neuroscience 22(6): 1112-1123.

Sterelny, K. (2003). Thought in a hostile world : the evolution of human cognition. Malden, MA, Blackwell.

Stewart, K. M. (1994). "Early Hominid Utilization of Fish Resources and Implications for Seasonality and Behavior." Journal of Human Evolution 27(1-3): 229-245.

Suddendorf, T. and M. C. Corballis (2007). "The evolution of foresight: What is mental time travel, and is it unique to humans?" Behavioral and Brain Sciences 30(03): 299-313.

Suddendorf, T., Corballis, Michael C. (1997). "Mental time Travel and the evolution of the human mind." Genetic, Social \& General Psychology Monographs 123(2): p133,35p.

Suwa, G., B. Asfaw, et al. (2009). "The Ardipithecus ramidus Skull and Its Implications for Hominid Origins." Science 326(5949): -

Suwa, G., R. T. Kono, et al. (2009). "Paleobiological Implications of the Ardipithecus ramidus Dentition." Science 326(5949): 69, 94-99.

Thierens, M., H. Pirlet, et al. "Ice-rafting from the British-Irish ice sheet since the earliest Pleistocene (2.6 million years ago): implications for long-term 
mid-latitudinal ice-sheet growth in the North Atlantic region." Quaternary Science Reviews In Press, Corrected Proof.

Thomas, E. M. (1963). "Bushmen of the Kalahari." National Geographic 123(6): 866-888.

Tobias, P. V. (1987). "The Brain of Homo-Habilis - a New Level of Organization in Cerebral Evolution." Journal of Human Evolution 16(78): 741-761.

Tomasello, M. (2008). Origins of human communication. Cambridge, Mass., MIT Press.

Tomasello, M., M. Carpenter, et al. (2005). "Understanding and sharing intentions: The origins of cultural cognition." Behavioral and Brain Sciences 28(5): 675-691.

Tomasello, M. and H. Rakoczy (2003). "What makes human cognition unique? From individual to shared to collective intentionality." Mind \& Language 18(2): 121-147.

Toth, N. and K. Schick (2009). "The Oldowan: The Tool Making of Early Hominins and Chimpanzees Compared." Annual Review of Anthropology 38: 289-305.

Tulving, E. (1985). "How Many Memory-Systems Are There." American Psychologist 40(4): 385-398.

Tuttle, R., D. Webb, et al. (1991). "Laetoli toes and\&lt;i\&gt;Australopithecus afarensis\&lt;/i\&gt." Human Evolution 6(3): 193-200.

Tuttle, R., D. Webb, et al. (1990). "Further progress on the Laetoli trails." Journal of Archaeological Science 17(3): 347-362.

Verhaegen, M., P. F. Puech, et al. (2002). "Aquarboreal ancestors?" Trends in Ecology \& Evolution 17(5): 212-217.

Wadley, L. (2010). "Were snares and traps used in the Middle Stone Age and does it matter? A review and a case study from Sibudu, South Africa." Journal of Human Evolution 58(2): 179-192.

West-Eberhard, M. J. (2005). "Developmental plasticity and the origin of species differences." Proceedings of the National Academy of Sciences of the United States of America 102(Suppl 1): 6543-6549.

White, T. D., B. Asfaw, et al. (2009). "Ardipithecus ramidus and the Paleobiology of Early Hominids." Science 326(5949): 75-86.

Whiten, A. (2000). "Primate culture and social learning." Cognitive Science 24(3): 477-508.

Whiten, A. (2008). "Imitation, emulation, and the transmission of culture." Behavioral and Brain Sciences 31(01): 39-40.

WoldeGabriel, G., S. H. Ambrose, et al. (2009). "The Geological, Isotopic, Botanical, Invertebrate, and Lower Vertebrate Surroundings of Ardipithecus ramidus." Science 326(5949): 65, 65e1-65e5. 
Wrangham, R. and N. Conklin-Brittain (2003). "[']Cooking as a biological trait'." Comparative Biochemistry and Physiology - Part A: Molecular \& Integrative Physiology 136(1): 35-46.

Wynn, T. (2002). "Archaeology and cognitive evolution." Behavioral and Brain Sciences 25(03): 389-402.

Zollikofer, C. P. E. and M. S. Ponce de León "The evolution of hominin ontogenies." Seminars in Cell \& Developmental Biology 21(4): 441-452. 